OAK RIDGE

NATIONAL LABORATORY

\title{
Thermal Hydraulic Characteristics of Fuel Defects in Plate Type Nuclear Research Reactors
}

May 2014

I. T. Bodey

R. V. Arimilli

J. D. Freels

Approved for public release;

distribution is unlimited.

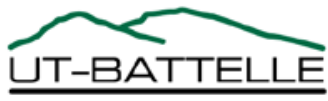




\section{DOCUMENT AVAILABILITY}

Reports produced after January 1, 1996, are generally available free via the U.S. Department of Energy (DOE) Information Bridge.

Web site http://www.osti.gov/bridge

Reports produced before January 1, 1996, may be purchased by members of the public from the following source.

National Technical Information Service

5285 Port Royal Road

Springfield, VA 22161

Telephone 703-605-6000 (1-800-553-6847)

TDD 703-487-4639

Fax 703-605-6900

E-mail info@ntis.gov

Web site http://www.ntis.gov/support/ordernowabout.htm

Reports are available to DOE employees, DOE contractors, Energy Technology Data Exchange (ETDE) representatives, and International Nuclear Information System (INIS) representatives from the following source.

Office of Scientific and Technical Information

P.O. Box 62

Oak Ridge, TN 37831

Telephone 865-576-8401

Fax 865-576-5728

E-mail reports@osti.gov

Web site http://www.osti.gov/contact.html

This report was prepared as an account of work sponsored by an agency of the United States Government. Neither the United States Government nor any agency thereof, nor any of their employees, makes any warranty, express or implied, or assumes any legal liability or responsibility for the accuracy, completeness, or usefulness of any information, apparatus, product, or process disclosed, or represents that its use would not infringe privately owned rights. Reference herein to any specific commercial product, process, or service by trade name, trademark, manufacturer, or otherwise, does not necessarily constitute or imply its endorsement, recommendation, or favoring by the United States Government or any agency thereof. The views and opinions of authors expressed herein do not necessarily state or reflect those of the United States Government or any agency thereof. 


\title{
Thermal Hydraulic Characteristics of Fuel Defects in Plate Type Nuclear Research Reactors
}

\author{
A Dissertation Presented for the \\ Doctor of Philosophy \\ Degree \\ The University of Tennessee, Knoxville
}

Isaac Thomas Bodey

May 2014 


\section{Dedication}

This work is dedicated to my wife Danielle and daughter Olivia for their patience, understanding, and support during its conception and execution. I would be lost without them.

I would like to thank the rest of my family, especially my mother Kristine, step-father Kent, and father Wainty for helping me along the way.

I would also like to thank the gentlemen that constitute the "Table of Knowledge" which resides within the confines of trailer 7964C, the most expensive triple-wide trailer in Eastern Tennessee. 


\section{Acknowledgements}

I would like to thank Dr. Rao Arimilli for giving me the opportunity to perform doctoral research in thermal-fluid mechanics and for the many hours spent discussing issues in turbulent flow. I want to acknowledge Dr. Arimilli's dedication to the education of engineers in thermal science and the progression of original ideas regarding scientific inquiry. Thanks for listening to my mind wander.

I would like to thank Dr. Majid Keyhani for his unparalleled knowledge of convection heat transfer and being one of the most effective instructors that I have met. Thanks for the advice and constructive criticism. "In experimental work, you can't tell material properties, 'Thou shalt be constant!"”

I would like to thank Dr. Kivanc Ekici, Dr. Arthur Ruggles, Dr. James D. Freels, and Dr. David Cook for their time, advice, and service on this committee. Their input has been and will always be invaluable. 


\begin{abstract}
Turbulent flow coupled with heat transfer is investigated for a High Flux Isotope Reactor (HFIR) fuel plate. The Reynolds Averaged Navier-Stokes Models are used for fluid dynamics and the transfer of heat from a thermal nuclear fuel plate using the Multi-physics code COMSOL. Simulation outcomes are compared with experimental data from the Advanced Neutron Source Reactor Thermal Hydraulic Test Loop. The computational results for the High Flux Isotope Reactor core system provide a more physically accurate simulation of this system by modeling the turbulent flow field in conjunction with the diffusion of thermal energy within the solid and fluid phases of the model domain. Recommendations are made regarding Nusselt number correlations and material properties for future thermal hydraulic modeling efforts.
\end{abstract}




\section{Table of Contents}

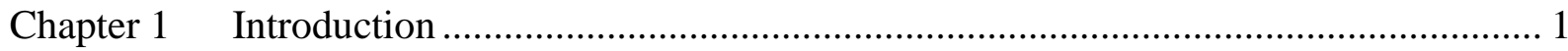

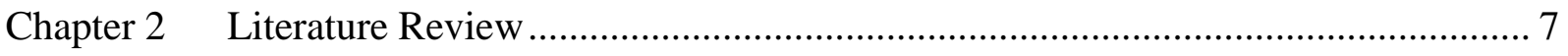

2.1 Review of Legacy Code Characteristics ............................................................... 8

2.2 Review of Fuel Defect Modeling ................................................................. 13

2.3 Review of the Advanced Neutron Source Reactor Thermal Hydraulic Test Loop .... 17

2.4 Motivation and Objectives for This Dissertation ........................................... 18

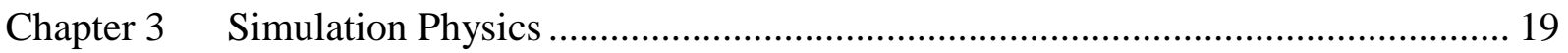

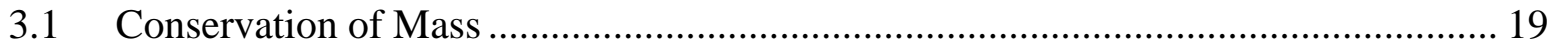

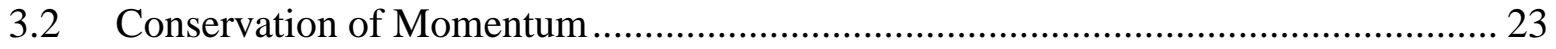

3.2.1 The k- $\omega$ Closure Model ............................................................................... 26

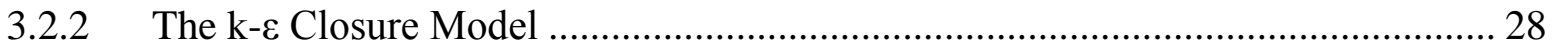

3.2.3 The Low Reynolds Number k- $\varepsilon$ Model ......................................................... 29

3.2.4 The Spallart-Allmaras Model ................................................................. 30

3.2.5 Turbulent Model Closure Discussion .............................................................. 31

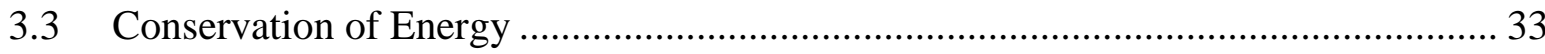

3.3.1 Energy Conservation Principle Applied to the Flow Field ................................... 34

3.3.2 Energy Conservation Principle Applied to Solids ............................................ 39

Chapter 4 2-D Model Development: Turbulent Conjugate Heat Transfer (TCHT) ............ 41

4.1 2-D TCHT Model Geometry and Computational Grid Structure............................ 41

4.2 2-D Turbulent Conjugate Heat Transfer (TCHT) Analysis: Constant Heat Flux case with Constant and Variable Fluid Properties. ............................................................. 49

4.3 2-D Turbulent Conjugate Heat Transfer: HFIR Distributed Power Density Case with Constant and Variable Fluid Properties ........................................................................ 68 
4.4 Conclusions for the 2-D Modeling Case 84

Chapter 5 3-D Model Development: Advanced Neutron Source Reactor Thermal Hydraulic

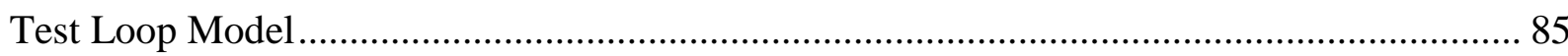

5.1 ANSR THTL Model Geometry and Boundary Conditions ..................................... 86

5.2 Computational Grid Structure Development .................................................... 89

5.3 Comparison of Model Results with Experimental Data ..................................... 90

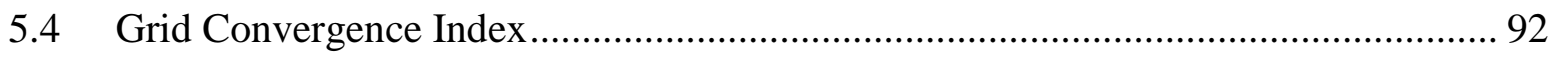

Chapter 6 3D Model Development: Thermal Hydraulic Characteristics of Fuel Defects

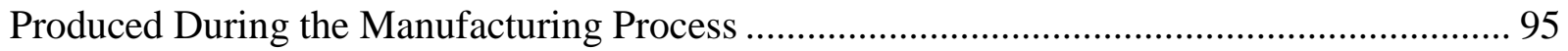

6.1 Plate Geometry and Grid Structure ............................................................. 95

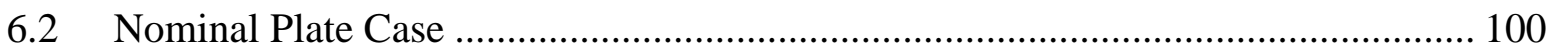

6.3 Blister Defect Case .................................................................................... 105

6.4 Segregation Defect Case ...................................................................... 110

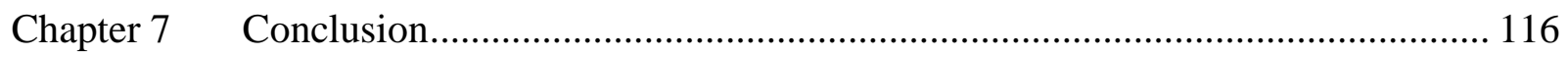

$7.1 \quad$ Results from 2-D Models ..................................................................... 116

7.2 Results from the 3-D Models................................................................... 117

7.2.1 Advanced Neutron Source Reactor Thermal Hydraulic Test Loop .................... 118

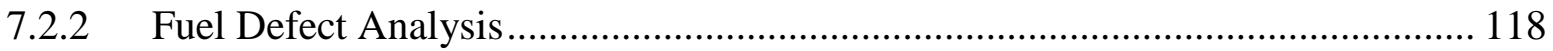

7.3 Considerations for Future Use of Material in this Dissertation ............................ 119

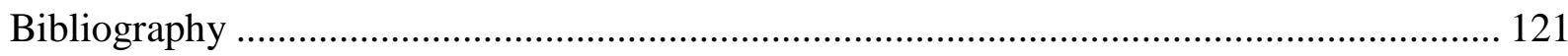

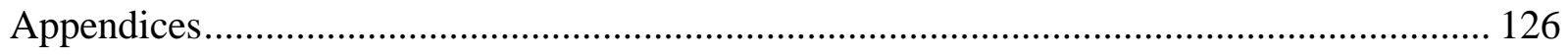

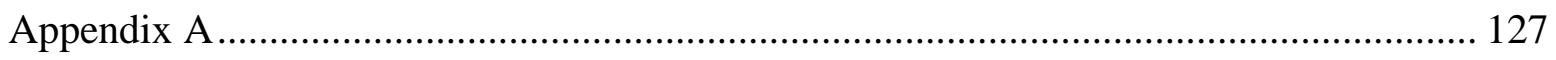

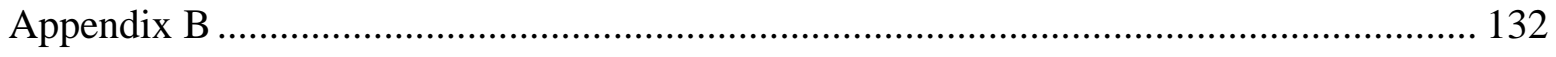

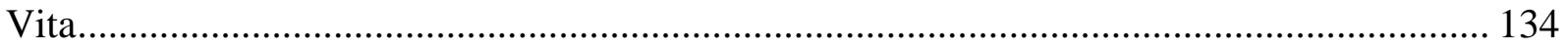




\section{List of Figures}

Figure 1-1. Cutaway View of the HFIR Core [1] ................................................................. 2

Figure 1-2. Axial View of the Radial Cross-section of the HFIR Inner Fuel Element Fuel Plate [7].

Figure 2-1. Fuel Plate Surface Discretization used in the SSHTC [14].................................... 10

Figure 2-2. Power Density Distribution Array. ...................................................................... 12

Figure 2-3. Axisymmetric Geometry used for Fuel Defect Simulations [13] [15]..................... 15

Figure 3-1. The Effect of Wall-Offset Models with Grid Elements Too Close to the Wall........ 32

Figure 4-1. 2-D Turbulent Conjugate Heat Transfer Model Geometry ....................................... 43

Figure 4-2. 2-D Turbulent Conjugate Heat Transfer Model Geometry ........................................ 44

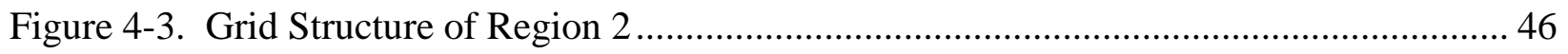

Figure 4-4. Temperature Difference Between the Clad Surface and the Bulk Fluid for Constant Fluid Properties (CFP) and NIST Fluid Properties (NFP) ...................................................... 55

Figure 4-5. Convection Coefficient for Constant Fluid Properties (CFP) and NIST Fluid

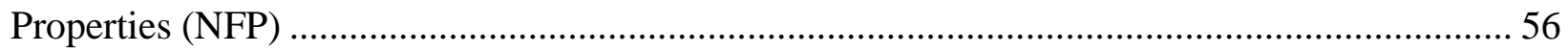

Figure 4-6. Difference between the Fluid Temperature and the Inlet Bulk Fluid Temperature for the Constant Fluid Property Case at Various Locations Along the Heated Section of the

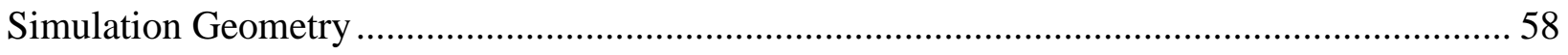

Figure 4-7. Difference between the Fluid Temperature and the Inlet Bulk Fluid Temperature for the Variable Fluid Property Case at Various Locations Along the Heated Section of the

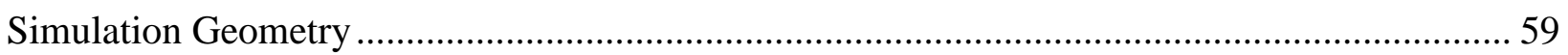

Figure 4-8. Difference between the CFP Fluid Temperature Distribution and the NFP Fluid Temperature Distribution at Different Axial Locations............................................................. 60 
Figure 4-9. Clad Surface Temperatures and Bulk Fluid Temperatures for both the CFP case and NFP case. 62

Figure 4-10. Comparison of the Nusselt Modulus - Prandtl Number Ratio as a Function of the Reynold's Number. 66

Figure 4-11. Relative Differences of the Gnielinski, Sieder-Tate, and Hausen Correlations compared with the (COMSOL) Model Nusselt Modulus.

Figure 4-12. Difference Between the Surface Temperature and the Inlet Bulk Fluid Temperature as Derived from the Correlations used for the CFP case. 69

Figure 4-13. Difference Between the Surface Temperature and the Bulk Fluid Inlet Temperature as Derived from the Nusselt Correlations used for the NFP case ........................................... 70 Figure 4-14. Distributed Power Density (DPD) Profile...................................................... 71 Figure 4-15. HFIR DPD Surface Heat Flux Distribution. 73 Figure 4-16. Profiles of the Difference between the Surface Temperature and the Bulk Fluid Temperature 74

Figure 4-17. Convection Coefficient for the CFP case and the NFP case. 75 Figure 4-18. Nusselt Number Correlation Results Compared with the Model Nusselt Number for the NFP. 78

Figure 4-19. Relative Differences of the Gnielinski, Sieder-Tate, and Hausen Correlations compared with the Model Nusselt Modulus. 80

Figure 4-20. Temperature Distribution for the CFP case Generated by the Gnielinski (G-CFP), Sieder-Tate (ST-CFP), and the Hausen (H-CFP) Correlations Compared with the Model Temperature Distribution (C-CFP). 82

Figure 4-21. Correlation Generated Temperature Distributions Compared with the Model Distribution for the NFP Case. 83

Figure 5-1. ANSR THTL Test Loop Cross Section ........................................................... 87

Figure 5-2. Isometric View of the ANSR THTL Model. ................................................. 88

Figure 6-1. Cross-sectional view of the fuel defect model geometry................................... 96 
Figure 6-2. The 3-D Power Density Profile used in the Fuel Meat Domain of the Simulation Geometry......

Figure 6-3. Nominal Case Clad Surface Temperature Along the Uncontoured Side of the Fuel

Plate. 101

Figure 6-4. Nominal Case Clad Surface Heat Flux Along the Uncontoured Side of the Fuel

Plate. 103

Figure 6-5. Blister Case Clad Surface Temperature Along the Uncontoured Side of the Fuel

Plate. 108

Figure 6-6. Blister Case Clad Surface Heat Flux Along the Uncontoured Side of the Fuel Plate. 109

Figure 6-7. Clad Surface Temperature on the Fuel Contour Side of the Fuel Plate. 113

Figure 6-8. Clad Surface Heat Flux on the Fuel Contour Side of the Fuel Plate 113

Figure A-1. Thermal Conductivity of Water as a Function of the Thermodynamic State. ....... 128

Figure A-2. Isobaric Specific Heat of Water as a Function of the Thermodynamic State. ....... 129

Figure A-3. Dynamic Viscosity of Water as a Function of the Thermodynamic State............ 130

Figure A-4. Mass Density of Water as a Function of the Thermodynamic State. 131 


\section{Nomenclature}

\section{$\underline{\text { Symbol }}$}

$A_{c}$

A

Al

$B_{4} C$

C

$D_{h}$

Ele\#

En

$\boldsymbol{F}, F_{i}$

$f$

$\tilde{h}$

$i, j$

$\overline{\bar{k}}, k_{i j}$

$k_{f}$

$k_{\text {turb }}$

$L$

l
Definition

Cross-sectional Area

Total Heat Transfer Area

Chemical Element Aluminum

Tetra-Boron Carbide

Specific Heat Capacity

Hydraulic Diameter

Number of Finite Elements

Enrichment

Body Force per Unit Volume

Fraction of Reactor Heat Deposited

in the Fuel Meat

Favre Averaged Enthalpy

Grid Indices for $\Omega \in R^{2}$

Thermal Conductivity Tensor

Fluid Thermal Conductivity

Turbulent Kinetic Energy per unit mass

Total Length of Domain

Length of Lateral Span of the

Fuel Plate

\section{Units}

in. $^{2}\left(\mathrm{~m}^{2}\right)$

in. $^{2}\left(\mathrm{~m}^{2}\right)$

$\mathrm{Btu} / \mathrm{lb}_{\mathrm{m}} /{ }^{\circ} \mathrm{F}(\mathrm{J} / \mathrm{kg} / \mathrm{K})$

in. (m)

1

1

$\mathrm{lb}_{\mathrm{f}} / \mathrm{in}^{3}{ }^{3}\left(\mathrm{~N} / \mathrm{m}^{3}\right)$

1

$\mathrm{Btu} / \mathrm{lb} \mathrm{m}_{\mathrm{m}}(\mathrm{J} / \mathrm{kg})$

$\mathrm{Btu} / \mathrm{hr} / \mathrm{ft} /{ }^{\circ} \mathrm{F}(\mathrm{W} / \mathrm{m} / \mathrm{K})$

$\mathrm{Btu} / \mathrm{hr} / \mathrm{ft} /{ }^{\circ} \mathrm{F}(\mathrm{W} / \mathrm{m} / \mathrm{K})$

$\mathrm{ft}^{2} / \mathrm{s}^{2}\left(\mathrm{~m}^{2} / \mathrm{s}^{2}\right)$

in. (m)

in. (m) 


\section{$\underline{\text { Symbol }}$}

$L_{b}$

$\dot{m}$

$N u_{D}$

Pr

$P_{w}$

$q^{\prime \prime \prime}$

$Q_{p}$

$q_{i, j}^{\prime \prime}$

$r$

Re

$\mathbb{R}^{N}$

$t$

$T$

$T_{\text {sat }}$

$\Delta T_{i, j}^{b u l k}$

$T_{S}$

$T_{\text {bulk }}$

$U$
Definition

Domain Boundary Length

Mass Flow Rate

Nusselt Number Based on

Hydraulic Diameter

Prandtl Number

Wetted Perimeter

Power Density

Total Reactor Power

Nodal Heat Flux

Position Vector

Reynolds Number

Set of Real Numbers with

Dimensionality N

Thickness of Fuel Plate

Temperature

Saturation Temperature of Water

Nodal Bulk Water Temperature

Clad Surface Temperature

Bulk Water Temperature

Chemical Element Uranium $\underline{\text { Units }}$

in. (m)

$\mathrm{lb}_{\mathrm{m}} / \mathrm{s}(\mathrm{kg} / \mathrm{s})$

1

1

in. (m)

$\mathrm{Btu} / \mathrm{hr} / \mathrm{ft}^{3}\left(\mathrm{~W} / \mathrm{m}^{3}\right)$

$\mathrm{Btu} / \mathrm{hr}(\mathrm{W})$

$\mathrm{Btu} / \mathrm{hr} / \mathrm{ft}^{2}\left(\mathrm{~W} / \mathrm{m}^{2}\right)$

in. (m)

1

in. ${ }^{N}\left(m^{N}\right)$

in. (m)

${ }^{\circ} \mathrm{F}(\mathrm{K})$

${ }^{\circ} \mathrm{F}(\mathrm{K})$

${ }^{\circ} \mathrm{F}(\mathrm{K})$

${ }^{\circ} \mathrm{F}(\mathrm{K})$

${ }^{\circ} \mathrm{F}(\mathrm{K})$ 


\section{Symbol}

$\widetilde{\boldsymbol{u}}, \widetilde{u_{\imath}}$

$\boldsymbol{u}^{\prime \prime}, u_{i}^{\prime \prime}$

$|\bar{V}|$

Vector

w

$x, x_{1}$

$y, x_{2}$

$z, x_{3}$

\section{Greek Symbols}

$\delta_{i j}$

$\epsilon$

$\mu$

$\mu_{T}$

$\mu_{w}$

$\rho$

$\rho_{b}$

$\sigma^{*}$

$\phi_{i, j}$
Definition

Favre Averaged Velocity Vector

Velocity Fluctuation

Magnitude of the Average Velocity

$\underline{\text { Units }}$

fps $(\mathrm{m} / \mathrm{s})$

fps $(\mathrm{m} / \mathrm{s})$

fps $(\mathrm{m} / \mathrm{s})$

in. (m)

in. (m)

for $\Omega \in R^{3}$. Axial Direction in

Cartesian Frame for $\Omega \in R^{2}$

Normal Direction to Fuel Plate Clad

in. $(\mathrm{m})$

Axial Direction in Cartesian Frame

in. $(\mathrm{m})$

for $\Omega \in R^{3}$

Kronecker Delta

Dissipation of turbulent kinetic energy Btu/s (J/s)

Dynamic Viscosity $\quad \mathrm{lb}_{\mathrm{m}} / \mathrm{ft} / \mathrm{hr}\left(\mathrm{Pa}^{*} \mathrm{~s}\right)$

Eddy Viscosity

$\mathrm{lb}_{\mathrm{m}} / \mathrm{ft} / \mathrm{hr}\left(\mathrm{Pa}^{*} \mathrm{~s}\right)$

Dynamic Viscosity at Solid-Fluid

$\mathrm{lb}_{\mathrm{m}} / \mathrm{ft} / \mathrm{hr}\left(\mathrm{Pa}^{*} \mathrm{~s}\right)$

Boundary

Mass Density

$\mathrm{lb}_{\mathrm{m}} / \mathrm{ft}^{3}\left(\mathrm{~kg} / \mathrm{m}^{3}\right)$

Boundary Element Density

Elements per unit length

k- $\omega$ Turbulence Model Closure Constant

1

Power Density Distribution Array 
Symbol

$\Omega$

$\omega$
Definition

Set of Points Representing the

Physical Domain

Specific Turbulence Dissipation Rate $\underline{\text { Units }}$ 


\section{Chapter 1 Introduction}

The High Flux Isotope Reactor (HFIR) is a light water moderated and cooled $100 \mathrm{MW}$ rated research reactor located at the Oak Ridge National Laboratory (ORNL) in Oak Ridge, Tennessee. Currently, the HFIR operates at a reduced power of $85 \mathrm{MW}$. The reduction in operational power was a result of a necessary decrease in system pressure due to the embrittlement of the pressure vessel that occurred during the initial 20 years of operation of the device. The HFIR had, and continues to have, the highest neutron flux in the western hemisphere [1]. For the rated operational power of $100 \mathrm{MW}$, the thermal-neutron flux in the flux trap region, i.e. the center of the core, is $5.5 \times 10^{15}$ neutrons $/ \mathrm{cm}^{2} / \mathrm{s}$. For the reduced power of 85 MW, the thermal-neutron flux in the flux trap is $2.6 \times 10^{15}$ neutrons $/ \mathrm{cm}^{2} / \mathrm{s}$. Thermal-neutrons are defined as those neutrons that have undergone a sufficient number of collisions with other material particles such that their speeds are comparable to the thermal motion of the atoms in the moderator material, i.e. thermal equilibrium is achieved between the free neutrons and the moderator material with energies of approximately $0.025 \mathrm{eV}[2]$.

The original purpose of the HFIR system was to produce trans-plutonium isotopes, specifically californium-252. The HFIR has many experimental facilities available for isotope production, i.e. locations within and surrounding the core, such as the already mentioned flux trap, the removable beryllium locations which surround the core, and control rod access plug facilities [1]. The HFIR also has four neutron beam tubes used to direct the neutrons to targets for both elastic and inelastic scattering experiments used in the determination of the structural and excited properties of materials, respectively.

The HFIR consists of two concentric annular fuel elements. A cutaway view of the HFIR core is shown in Figure 1-1. The inner fuel element consists of 171 fuel plates while the outer fuel element consists of 369 fuel plates. Each fuel plate is nominally $24 \mathrm{in}$. (0.6096 m) long in the axial direction and 0.05 in. $(1.27 \mathrm{~mm})$ thick. Thus the aspect ratio, length to thickness, is very high with a magnitude of 480 . The lateral measure, i.e. perpendicular to both the axial direction and 


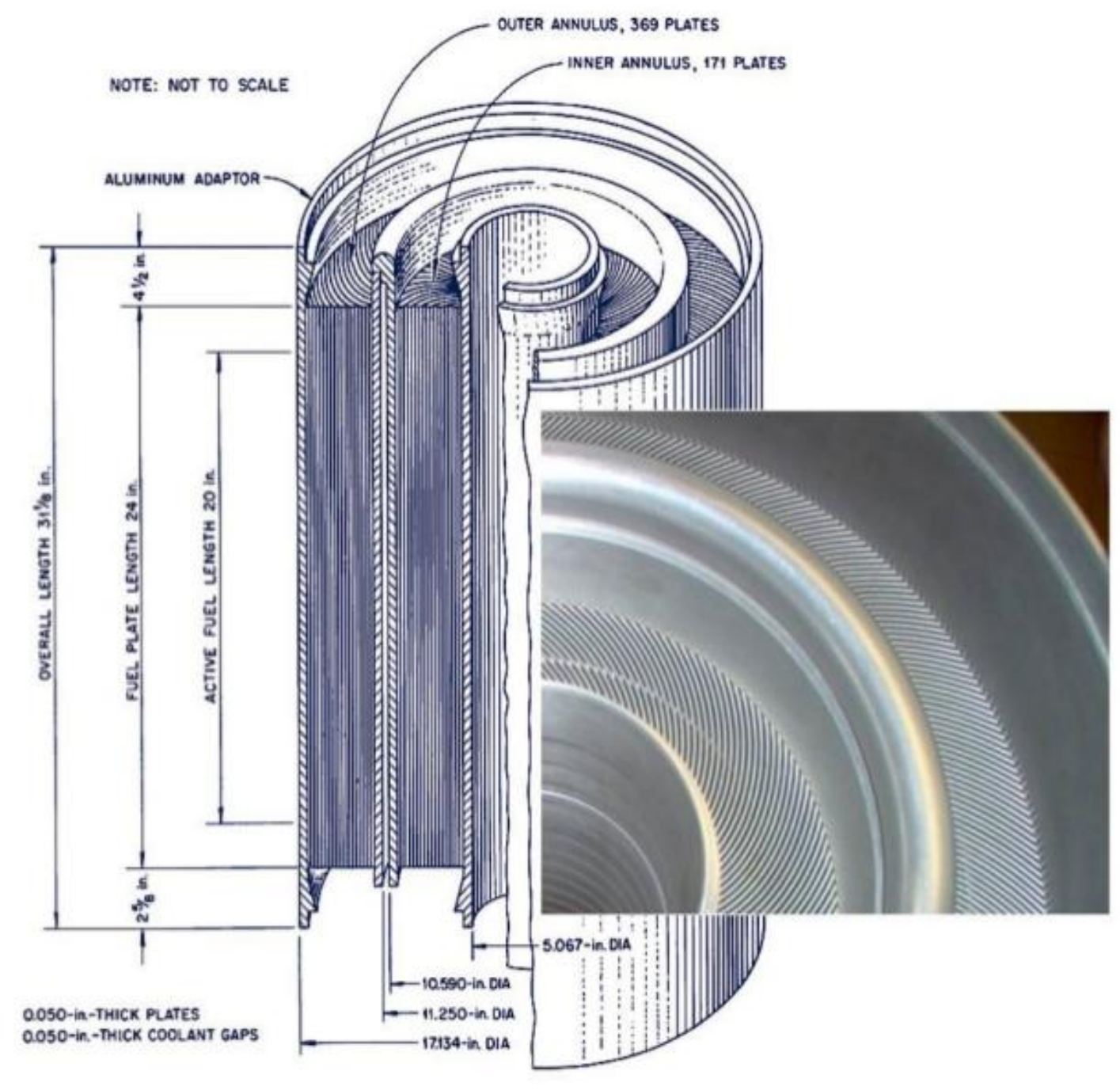

Figure 1-1. Cutaway View of the HFIR Core [1]. The inset is a photograph of the fuel elements. 
the thickness, of the fuel plate is different for the two elements. The inner element fuel plates have a lateral length of $0.276 \mathrm{ft} .(0.0841 \mathrm{~m})$ and the outer fuel element fuel plates have a lateral length of $0.245 \mathrm{ft}$. $(0.0747 \mathrm{~m})$ [3]. Two adjacent fuel plates, in both the inner and outer fuel elements, form a 0.05 in. $(1.27 \mathrm{~mm})$ coolant flow channel with the same aspect ratio as the fuel plate.

The HFIR currently uses a highly enriched uranium (HEU) fuel. The fuel is characterized by the combination of a $\mathrm{U}_{3} \mathrm{O}_{8}-\mathrm{Al} 1100$ packed powder cermet with an enrichment of $93 \%{ }^{235} \mathrm{U}$, i.e. the fuel meat, and $\mathrm{B}_{4} \mathrm{C}-\mathrm{Al} 1100$ filler. Enrichment is defined as the ratio of the mass of ${ }^{235} \mathrm{U}$ to the total amount of uranium in the $\mathrm{U}_{3} \mathrm{O}_{8}$ material, i.e.

$E n \equiv \frac{{ }^{235} U}{U}$

A radial cross-section of the fuel plate is shown in Figure 1-2. The geometry of the fuel plate is that of a circular involute with the fuel meat contoured relative to this base involute geometry. The fuel, i.e. fuel meat and filler combination, forms a constant area cross-section with a thickness of 0.030 in. $\left(7.62 \times 10^{-4} \mathrm{~m}\right)$ hermetically sealed within the Al 6061 clad. The fuel extends axially through the center 20.0 in. $(0.508 \mathrm{~m})$ of the clad as shown in Figure 1-1. The combination of the fuel and the clad forms a single fuel plate.

In an effort to reduce the proliferation of nuclear materials, the global threat reduction initiative (GTRI) was implemented with the mission of reducing and protecting nuclear and radiological materials in civilian sites according to the National Nuclear Security Administration [4]. To comply with the GTRI, the Department of Energy (DOE) initiated the Reduced Enrichment for Research and Test Reactors (RERTR) program in 1978 [5]. This program develops the technology that allows research and test reactors to convert to a low enriched uranium (LEU) fuel, i.e. 19.75 wt.\% ${ }^{235} \mathrm{U}$, in compliance with the GTRI.

The LEU fuel is a U-10Mo alloy, i.e. $90 \mathrm{wt} \% \%$ Uranium and $10 \mathrm{wt}$ \% Molybdenum, with an enrichment of 19.75 wt. $\%{ }^{235} \mathrm{U}$ [6]. In order to maintain the same thermal neutron flux found with the HEU fuel without changing the core geometry, the LEU fuel must be much 


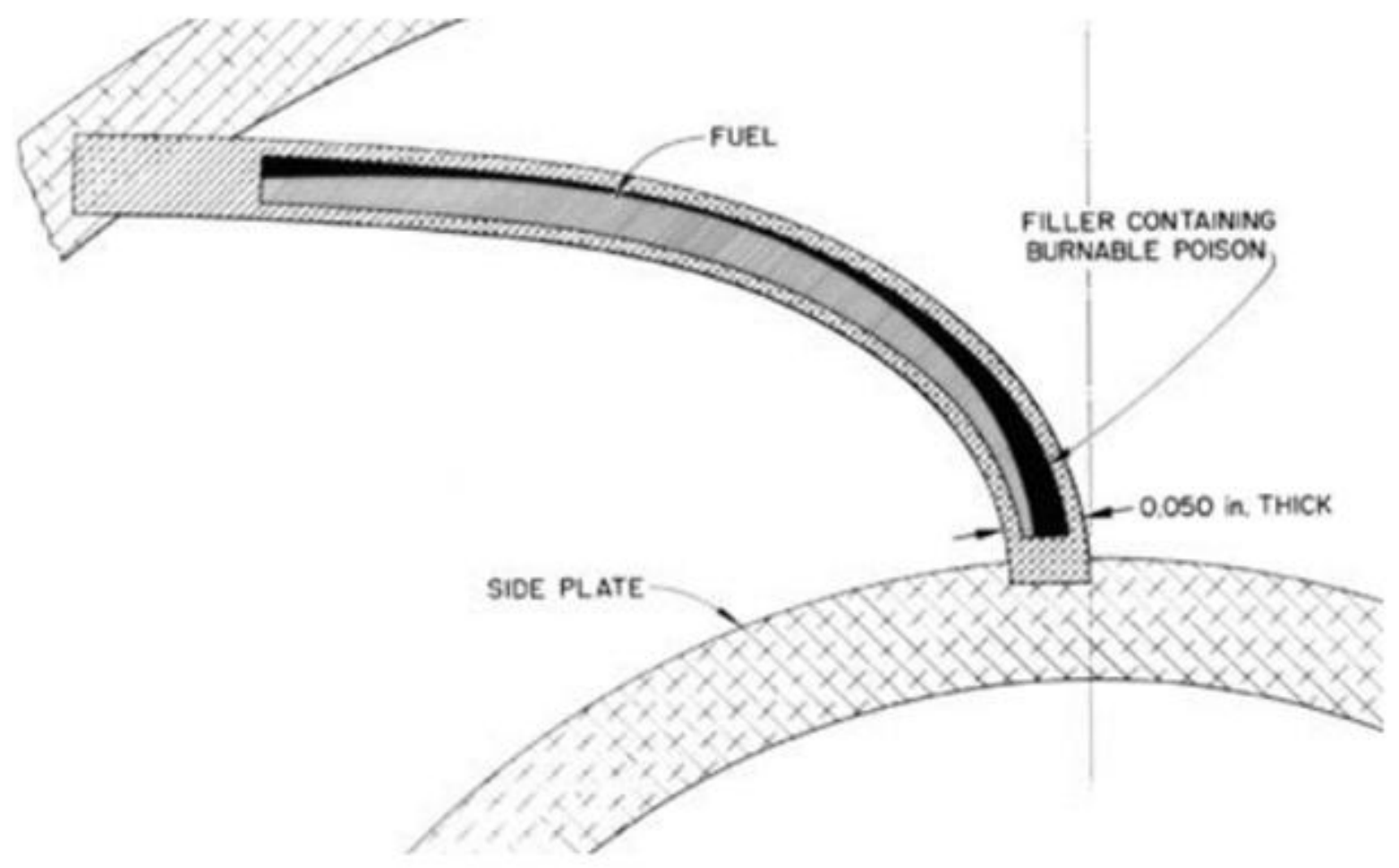

Figure 1-2. Axial View of the Radial Cross-section of the HFIR Inner Fuel Element Fuel Plate [7]. 
more dense than the HEU fuel. As a result, the LEU fuel meat will be a contoured monolithic foil as opposed to a contoured packed powder LEU fuel meat similar to the HEU configuration that is currently in use.

It is important to note that the HFIR core geometry has remained unchanged during its 56 year operation as of this writing. It is currently mandated that the HFIR core outer geometry maintain its current configuration, however, the geometrical configuration within the fuel plates can, and will most likely, change with the proposed U-10Mo alloy, i.e. LEU, fuel.

The steady state flow rate of the coolant through the HFIR core is $13,413 \mathrm{gpm}$ [3]. This translates to a steady state coolant velocity of $52.00 \mathrm{fps}(15.85 \mathrm{~m} / \mathrm{s})$ through each individual 0.050 in. $(1.27 \mathrm{~mm})$ thick coolant channel. The inlet coolant temperature is $120.00{ }^{\circ} \mathrm{F}(322.04$ K) with an inlet fluid pressure of $482.7 \mathrm{psi}(3.328 \mathrm{MPa})$. As a result, the inlet cooling fluid density and dynamic viscosity are $61.800 \mathrm{lb} / \mathrm{ft}^{3}\left(989.94 \mathrm{~kg} / \mathrm{m}^{3}\right)$ and $1.3492 \mathrm{lb} / \mathrm{ft} / \mathrm{hr}$ $\left(5.5772 \times 10^{-4} \mathrm{~Pa}^{*} \mathrm{~s}\right)$, respectively [8].

The hydraulic diameter of the flow channel is approximated as twice the channel spacing due to the relative sizes of the channel spacing and the lateral length of the channel. The aspect ratio of the channel spacing, $t$, to the lateral length of the channel, $w$, is calculated to be 0.0015 . Since $t \ll w$, the hydraulic diameter can be expressed as

$D_{h}:=\frac{4 A_{c}}{P_{w}}=\frac{4 w t}{2(w+t)}=\frac{2 w t}{w\left(1+\frac{t}{w}\right)} \cong 2 t$.

Here $A_{c}$ is the cross-sectional area of the flow channel and $P_{w}$ is the wetted perimeter of the flow channel.

The inlet Reynolds number is calculated using the well known relationship

$$
R e=\frac{\rho|\overline{\boldsymbol{V}}| D_{h}}{\mu} .
$$


Here $\rho$ is the fluid density, $|\overline{\boldsymbol{V}}|$ is the average of the magnitude of the velocity at the inlet, and $\mu$ is the dynamic viscosity of the fluid.

Using the material properties of the fluid in conjunction with Equations 1.2 and 1.3, the channel inlet Reynolds number, $R e$, based on a hydraulic diameter of twice the channel spacing is 71,459 which is within the fully turbulent flow regime for internal flows, i.e. $R e \geq 10,000$ [9].

In what follows, rank one tensors, i.e. vectors, will be denoted by bold letters, $\boldsymbol{\phi}$, or a single subscripted index, $\phi_{i}$, while tensors of rank two or larger will be denoted by bold letters with a double over-bar, $\overline{\overline{\boldsymbol{\eta}}}$, or subscripted juxtaposed indices the number of which denoting the rank of the tensor, $\eta_{i j}$. Comma separated indices will be used to denote computational grid locations. Also, the Einstein summation convention will be employed throughout the document where tensor notation is used.

The objective of this work is to show, in a three dimensional space, how defects that occur within the fuel plate due to the manufacturing process establish perturbations in the thermal hydraulic characteristics of an entire fuel plate coupled with a flow channel system, and the results of those perturbations. Further, this work establishes a turbulent flow field which is responsible for the determination of the amount of heat removed from the fuel plate and as a result determines the values of the fluid material properties and the bulk temperature of the fluid. These aspects of the work are unique. 


\section{Chapter 2 Literature Review}

Early thermal hydraulic studies of the HEU HFIR core and its components were carried out using theoretical, numerical, and experimental analysis. These studies were performed for the design power of $100 \mathrm{MW}$. Many experiments were conducted in order to determine the thermal, hydraulic, and structural safety limits of the HFIR core and core components. Gambill and Bundy [10] performed experiments with diathermic turbulent flows in parallel channels. These experimental results were used to determine friction factors, burnout heat fluxes, and average and local Nusselt number correlations in support of the HFIR program. Cheverton and Sims [11] discuss critical experiments that were used in the design basis of the HFIR core. These experiments were conducted using different material types for the control rods and different core fuel loadings. Cheverton and Kelley [12] performed thermo-structural experiments to determine the expansion characteristics of the fuel plates due to thermal stresses. Data from these and other experimental programs were used in the development of nuclear safety simulations especially those performed by Hilvety and Chapman [13] and McLain [14]. The Steady State Heat Transfer code (SSHTC), developed by Hilvety and Chapman [13] and later modified by McLain [14], was and is still used to determine, numerically, safety limits of the reactor based on both experimental data and theoretical considerations for the original 100MW core and the more recent $85 \mathrm{MW}$ core. However, this code does not model the turbulent fluid flow, instead it uses a convection boundary condition with the convection coefficient determined by a modified version of the Hausen correlation for the Nusselt number. Further, the code calculates the change in the bulk fluid temperature, used in the convection boundary condition, by using "suitable heat balances" [14]. A further restriction imposed in the SSHTC is the constraint of diffusion of thermal energy to pass to the fluid in a direction normal to the clad surface only, i.e. thermal energy is not allowed to diffuse in axial and radial directions of the fuel plate.

Modern computational tools allow the user to create more physically accurate models of systems and system components. For example, in the case of the HFIR core, the ability to model the turbulent flow field provides a unique perspective of the thermal hydraulic characteristics of the fuel plate and the flow channels. This unique perspective is discussed in Chapter 6 where fuel defects and their thermal hydraulic consequences are investigated. While it is the case that 
the SSHTC provides sufficient information regarding the thermal hydraulic characteristics of the HFIR with HEU fuel, it is not commented adequately and difficult to modify. The proposed conversion of the HFIR to use an LEU fuel provides a unique opportunity to update the computational models used in the analysis of the core and indeed the entire system. As a result, the Research Reactors Division (RRD) of the Oak Ridge National Laboratory (ORNL) decided to use COMSOL for this effort. Version 4.3a of the COMSOL code was used for the computational work found herein. COMSOL provides a suite of finite element based multiphysics tools for use in the determination of physical quantities in a computational setting.

\subsection{Review of Legacy Code Characteristics}

The thermal hydraulic aspects of the SSHTC are representative of a Cartesian finite difference thermal diffusion code with $\Omega \in \mathbb{R}^{2}$ based upon the discretization of a flat fuel plate as shown in Figure 2-1 [14]. In Figure 2-1, the fueled region of the plate is enclosed by the heavy rectangle and the mesh points are denoted by comma separated subscripts $i, j$. The SSHTC is designed to be conservative to establish a safe thermal margin within which the HFIR HEU core can operate. The following discussion is a recapitulation of the thermal-fluid characteristics of the SSHTC and the conservative assumptions found therein.

The SSHTC does not account for axial or lateral thermal conduction of heat in the fuel plate material, i.e. the heat flux vector is strictly parallel to the plate surface area vector at each nodal location of the plate, i.e. $q^{\prime \prime} \cdot A=q " A$, [13]. As a result of this restriction on the heat flux, the code produces artificially high plate surface temperatures which are used as an extreme basis for the thermal limits of the physical reactor. These artificially high temperatures are considered extreme due to the reduction of the thermal margin consistent with approaching the saturation temperature of the fluid, $T_{\text {sat }}$. The saturation temperature along the fuel plate under current HFIR HEU operating conditions is

$$
438.88^{\circ} \mathrm{F}(499.19 \mathrm{~K}) \leq T_{\text {sat }} \leq 463.33^{\circ} \mathrm{F}(512.78 \mathrm{~K}) \text {. }
$$


This range is based upon a core pressure drop of $105 \mathrm{psi}(0.724 \mathrm{MPa})$ [3], where the larger value is indicative of upstream saturation temperatures due to the higher pressure experienced by the fluid.

The removal of thermal energy from the plate surface is performed in the SSHTC by the use of a modified version of the Hausen equation for the local Nusselt number correlation suggested by Gambill and Bundy as a result of their experimental work [10] [13] [14]. This relationship is shown in Equation 2.1 and is based on an earlier version of the correlation.

$N u_{D}=0.116\left(\operatorname{Re}_{z}^{2 / 3}-125\right) \operatorname{Pr}_{z}^{1 / 3}\left[1+\frac{1}{3}\left(\frac{D_{h}}{z}\right)^{2 / 3}\right]\left(\frac{\mu}{\mu_{w}}\right)_{z}^{0.14}$

Here $z$ represents the axial location along the plate surface where the fluid properties and dimensionless quantities are evaluated, $\operatorname{Pr}_{z}$ is the local Prandtl number of the flow, and $\mu_{w}$ is the dynamic viscosity taken at the solid-fluid interface.

The average correlation has since been modified by Hausen [15] and is shown in Equation 2.2.

$\overline{N u}_{D}=0.235\left(R e_{D}^{0.8}-230\right)\left(1.8 P r_{D}^{0.3}-0.8\right)\left[1+\left(\frac{D_{h}}{L}\right)^{2 / 3}\right]\left(\frac{\mu}{\mu_{w}}\right)^{0.14}$

The local Nusselt number derived from this relationship is the version that is currently used in the SSHTC for the 85MW core [16], which is shown in Equation 2.3.

$N u_{D}=0.0235\left(R e_{z}^{0.8}-230\right)\left(1.8 P r_{z}^{0.3}-0.8\right)\left[1+\frac{1}{3}\left(\frac{D_{h}}{z}\right)^{2 / 3}\right]\left(\frac{\mu}{\mu_{w}}\right)_{z}^{0.14}$

The numerical coefficient 0.0235, in Equation 2.3, is a correction performed by the author (Bodey) as it was discovered that the Hausen correlation reported in Thomas' work [16] was an order of magnitude larger than other correlations considered in this work, i.e. Sieder-Tate and Gnielinski. 


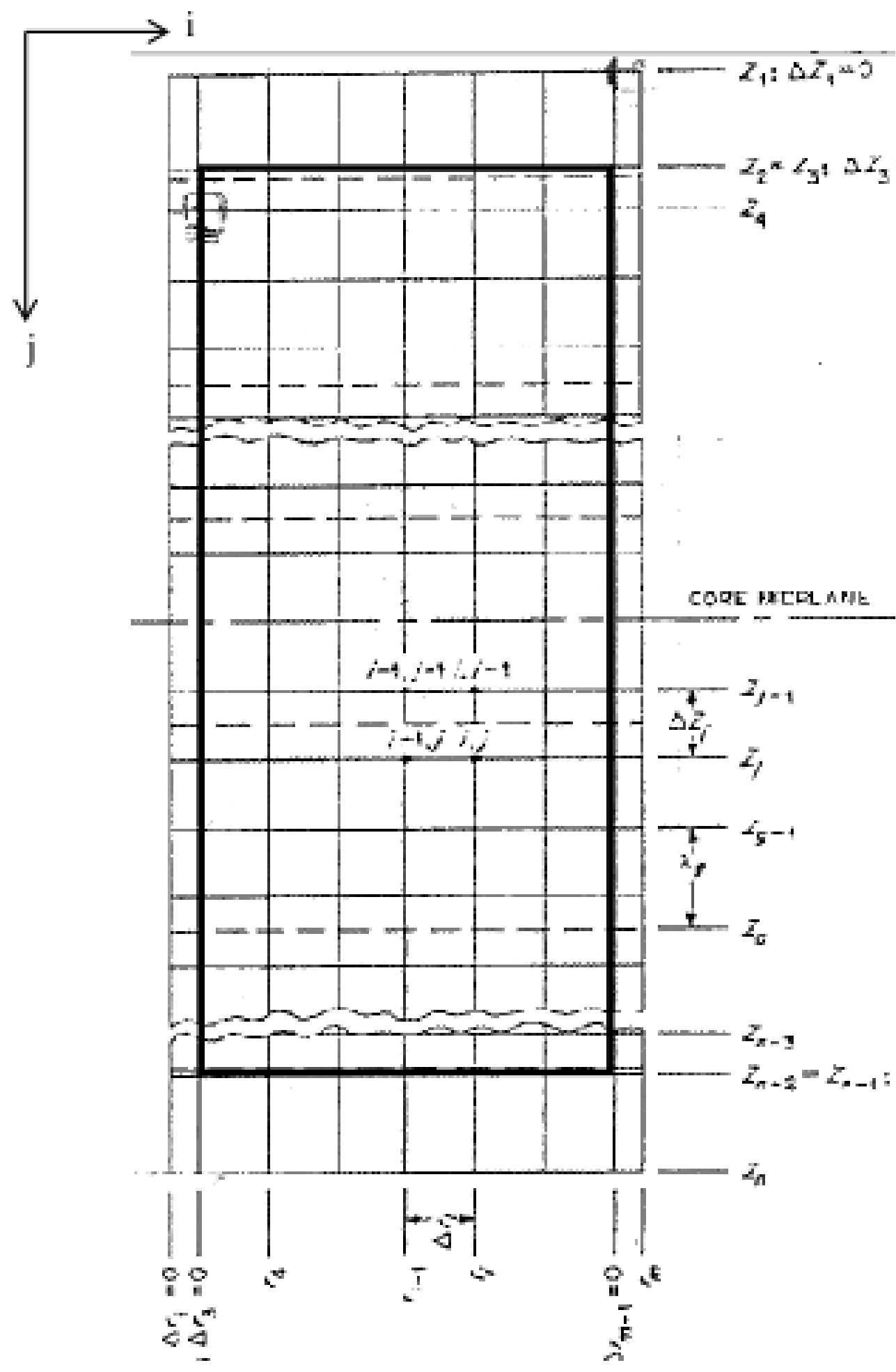

Figure 2-1. Fuel Plate Surface Discretization used in the SSHTC [14]. 
Since a Nusselt number correlation is employed in the code as a boundary condition for the convective heat flux, there is no need to simulate the non-isothermal turbulent flow physics that actually occurs in the system. This represents another limitation imposed on this simulation of the HFIR core system. At this point it is not clear whether the use of the Hausen equation represents a comparatively low value of the convection coefficient or not, however it will be shown later that it does indeed represent a value that increases clad surface temperatures. The other boundary conditions implemented in the SSHTC are an isothermal entrance set to $120^{\circ} \mathrm{F}(322.04 \mathrm{~K})$ with adiabatic side and exit boundaries [13].

The internal structure of the fuel plate is also not considered in the SSHTC simulation. Instead of considering the internal generation rate of thermal energy in the fuel due to the nuclear fission process, the heat flux at the fuel plate surface is calculated using a distribution array determined from nuclear fission density calculations using Monte Carlo methods. The development of this distribution array is not within the scope of this work.

The fuel plate surface heat flux at the $i, j$ location, refer to Figure $2-1$, is determined in the SSHTC by the following relationship.

$q_{i, j}^{\prime \prime}=\frac{Q_{p} f \phi_{i, j}}{A} \quad\{i, j \mid i=1, \cdots, 11 ; j=1, \cdots, 31\}$

Here $Q_{p}$ is the reactor power level, $f$ is a dimensionless quantity and is the fraction of reactor power deposited in the fuel meat, $\phi_{i, j}$ is the dimensionless power density distribution array, and $A$ is the total heat transfer area of the core. The power density distribution array, $\phi_{i, j}$, for the HEU inner fuel element is shown in Figure 2-2. The top and bottom two rows of the array, in Figure 2-2, represent the upper and lower 2.000in. $(0.0508 \mathrm{~m})$ of the fuel plate, i.e. the unfueled portion in the axial direction. The first and last two columns of the array, in Figure 2-2, represent the unfueled portion of the clad in the lateral direction.

The bulk temperature of the fluid is determined from "suitable heat balances" [14]. The equation used in the SSHTC to calculate the axial change in the bulk temperature at the node is 
$\begin{array}{lllllllllllll}0 & 0 & 0.000 & 0.000 & 0.000 & 0.000 & 0.000 & 0.000 & 0.000 & 0 & 0 \\ 0 & 0 & 0.000 & 0.000 & 0.000 & 0.000 & 0.000 & 0.000 & 0.000 & 0 & 0 \\ 0 & 0 & 0.678 & 1.109 & 1.379 & 1.515 & 1.470 & 1.344 & 1.186 & 0 & 0 \\ 0 & 0 & 0.722 & 0.741 & 0.738 & 0.771 & 0.809 & 0.830 & 0.837 & 0 & 0 \\ 0 & 0 & 0.815 & 0.753 & 0.738 & 0.767 & 0.802 & 0.830 & 0.824 & 0 & 0 \\ 0 & 0 & 0.924 & 0.848 & 0.810 & 0.821 & 0.850 & 0.889 & 0.893 & 0 & 0 \\ 0 & 0 & 1.031 & 0.952 & 0.904 & 0.917 & 0.939 & 0.980 & 0.988 & 0 & 0 \\ 0 & 0 & 1.130 & 1.043 & 0.990 & 1.007 & 1.027 & 1.067 & 1.077 & 0 & 0 \\ 0 & 0 & 1.227 & 1.126 & 1.068 & 1.089 & 1.107 & 1.146 & 1.162 & 0 & 0 \\ 0 & 0 & 1.312 & 1.198 & 1.137 & 1.163 & 1.179 & 1.217 & 1.241 & 0 & 0 \\ 0 & 0 & 1.387 & 1.264 & 1.200 & 1.233 & 1.241 & 1.280 & 1.316 & 0 & 0 \\ 0 & 0 & 1.447 & 1.322 & 1.255 & 1.285 & 1.294 & 1.334 & 1.383 & 0 & 0 \\ 0 & 0 & 1.493 & 1.372 & 1.306 & 1.332 & 1.339 & 1.380 & 1.445 & 0 & 0 \\ 0 & 0 & 1.520 & 1.398 & 1.338 & 1.369 & 1.372 & 1.410 & 1.478 & 0 & 0 \\ 0 & 0 & 1.532 & 1.408 & 1.350 & 1.386 & 1.388 & 1.422 & 1.490 & 0 & 0 \\ 0 & 0 & 1.533 & 1.403 & 1.349 & 1.386 & 1.388 & 1.420 & 1.485 & 0 & 0 \\ 0 & 0 & 1.523 & 1.393 & 1.335 & 1.372 & 1.375 & 1.418 & 1.464 & 0 & 0 \\ 0 & 0 & 1.494 & 1.368 & 1.305 & 1.342 & 1.345 & 1.380 & 1.425 & 0 & 0 \\ 0 & 0 & 1.448 & 1.324 & 1.256 & 1.289 & 1.295 & 1.330 & 1.372 & 0 & 0 \\ 0 & 0 & 1.384 & 1.264 & 1.193 & 1.221 & 1.235 & 1.283 & 1.312 & 0 & 0 \\ 0 & 0 & 1.312 & 1.195 & 1.127 & 1.150 & 1.167 & 1.212 & 1.248 & 0 & 0 \\ 0 & 0 & 1.235 & 1.120 & 1.057 & 1.077 & 1.097 & 1.144 & 1.177 & 0 & 0 \\ 0 & 0 & 1.148 & 1.039 & 0.982 & 1.000 & 1.023 & 1.069 & 1.098 & 0 & 0 \\ 0 & 0 & 1.050 & 0.952 & 0.903 & 0.920 & 0.945 & 0.988 & 1.011 & 0 & 0 \\ 0 & 0 & 0.944 & 0.859 & 0.820 & 0.839 & 0.864 & 0.904 & 0.916 & 0 & 0 \\ 0 & 0 & 0.819 & 0.762 & 0.735 & 0.755 & 0.783 & 0.816 & 0.812 & 0 & 0 \\ 0 & 0 & 0.709 & 0.668 & 0.667 & 0.698 & 0.735 & 0.762 & 0.753 & 0 & 0 \\ 0 & 0 & 0.706 & 0.678 & 0.680 & 0.713 & 0.749 & 0.760 & 0.762 & 0 & 0 \\ 0 & 0 & 0.703 & 1.028 & 1.231 & 1.342 & 1.319 & 1.216 & 1.078 & 0 & 0 \\ 0 & 0 & 0.000 & 0.000 & 0.000 & 0.000 & 0.000 & 0.000 & 0.000 & 0 & 0 \\ 0 & 0 & 0.000 & 0.000 & 0.000 & 0.000 & 0.000 & 0.000 & 0.000 & 0 & 0\end{array}$

Figure 2-2. Power Density Distribution Array. 
$\Delta T_{i, j}^{b u l k}=\frac{2 Q_{p} f}{A\left(\frac{\dot{m}}{l}\right)_{j}} \int_{0}^{z_{j}} \phi_{i, j} d z$.

where $\Delta T_{i, j}^{\text {bulk }}$ is the bulk temperature change, $\dot{m}$ is the mass flow rate, $l$ is the length of span of the plate, and $z_{j}$ is the axial location of the $j^{t h}$ node. There is a potential issue with this representation; the isobaric specific heat capacity, $C_{p}$, is not shown. The version of this equation that is used in the code is found further in the McLain document where a numerical constant, 6.584, multiplies the coefficient outside of the integral. It is suspected that this constant represents the isobaric specific heat capacity of the water, however, it is not explicitly stated in the documentation [14] how this constant was obtained. Hence, inadequate comments in the code are exemplified. Finally, the clad surface temperature, $T_{s}$, is found from Newton's law of cooling.

$T_{s}(r, z)=\frac{q^{\prime \prime}(r, z) z}{N u_{D} k_{f}}+T_{b u l k}(z)$

Here $k_{f}$ is the thermal conductivity of the fluid.

\subsection{Review of Fuel Defect Modeling}

Arguably, the most important aspect of the thermal hydraulic analysis has to do with the fuel defects that occur during the fuel plate manufacturing process that may not be detected by the subsequent inspection process. The defects considered are those associated with the lack of metallurgical bond between different interfaces on the interior of the fuel plate and that where the local quantity of ${ }^{235} \mathrm{U}$ is in excess of nominal values for the $\mathrm{U}_{3} \mathrm{O}_{8}-\mathrm{Al} 1100$ cermet but by no greater that $30 \%$ [14]. The lack of metallurgical bond is known as a blister or nonbond and the interesting locations within the fuel plate where this defect occurs are the fuel meat-clad 
interface, the filler-clad interface, and the fuel meat-filler interface. The local excessive quantity of ${ }^{235} \mathrm{U}$ is known as fuel segregation [17].

Hilvety \& Chapman [13], Kirkpatrick [17], and Giles [18] have studied the thermal consequences of these defects on the fuel plate materials using axisymmetric simulation geometries. The simulation geometry used in [13] and [17] is shown in Figure 2-3. Giles [18] uses a similar geometry, however the dimensions and the fuel meat components and locations are different as the analysis was based on the Advanced Neutron Source Reactor (ANSR) which was to use $\mathrm{U}_{3} \mathrm{Si}_{2}$ as the fuel instead of $\mathrm{U}_{3} \mathrm{O}_{8}$. It was determined by all investigators that the combination of the fuel defects in a single axial location at the interface of the fuel meat and filler, i.e. between the $\mathrm{U} 3 \mathrm{O} 8-\mathrm{Al} 1100$ and the $\mathrm{B}_{4} \mathrm{C}-\mathrm{Al} 1100$ materials, respectively, was considered "the most unfavorable situation" [13] [17] [18] due to the relatively high heat flux peaking factors calculated at this interfacial location as opposed to those calculated at the other interfacial locations within the fuel plate. For clarity, the heat flux peaking factor is defined by Hilvety \& Chapman [13] as the quantity by which the local heat flux produced by a fuel defect would need to be multiplied to achieve the incipient boiling heat flux for a specified power level. In mathematical terms the heat flux peaking factor is written as

$\alpha \equiv \frac{\left|\boldsymbol{q}_{i b}^{\prime \prime}\right|}{\left|\boldsymbol{q}_{\text {defect }}^{\prime \prime}\right|}$.

Here $\left|\boldsymbol{q}_{i b}^{\prime \prime}\right|$ is the magnitude of the incipient boiling heat flux and $\left|\boldsymbol{q}_{\text {defect }}^{\prime \prime}\right|$ is the magnitude of the local heat flux associated with the defect being considered.

The heat flux peaking factor is used in the SSHTC in the calculation of the "hotspot uncertainty factor", shown in Equation 2.8. 


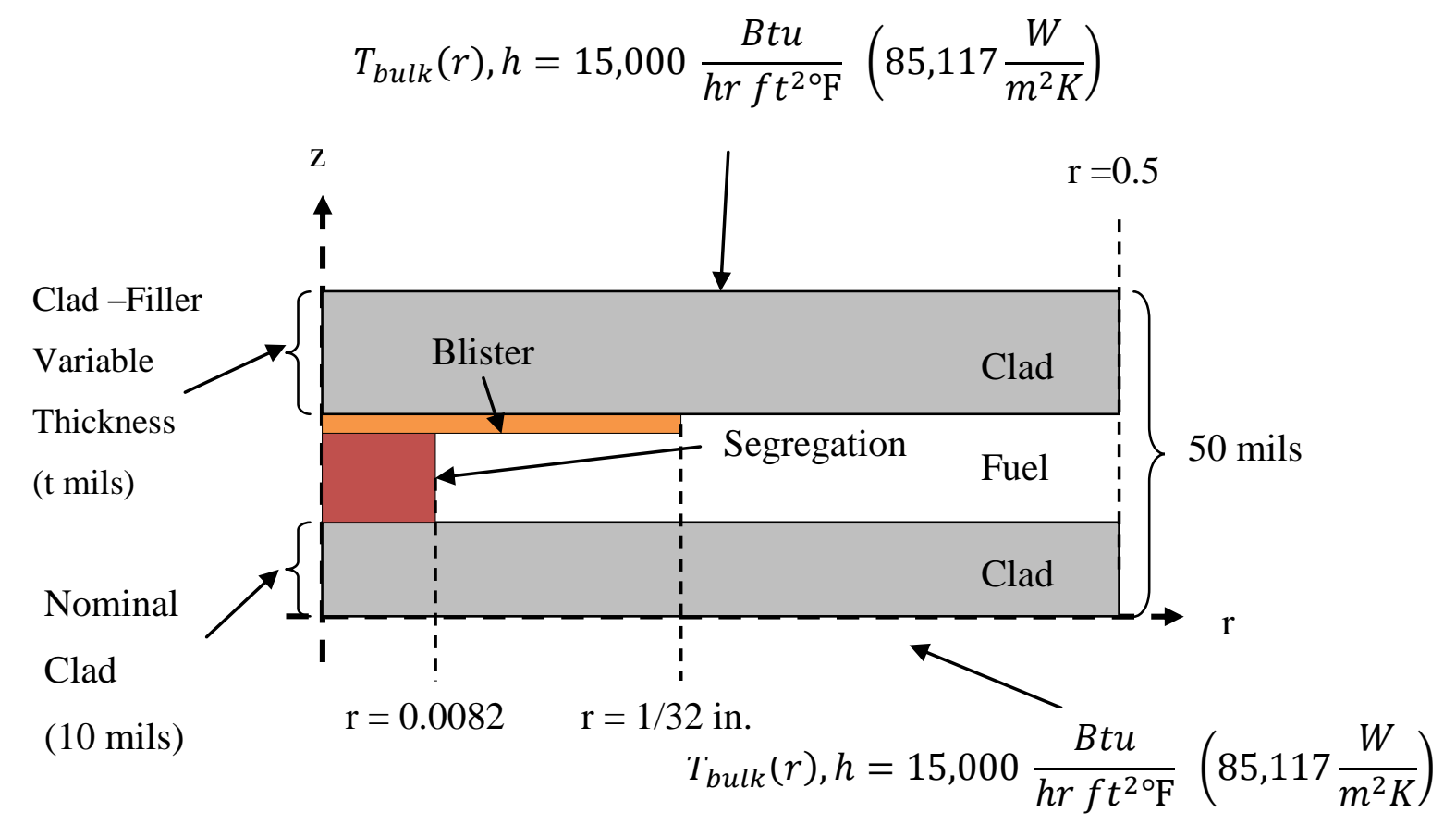

\begin{tabular}{|c|c|c|}
\hline Region & $\begin{array}{c}\text { Thermal Conductivity } \\
{\left[\frac{B T U}{h r f t^{\circ} \mathrm{F}}\right],\left(\frac{W}{m K}\right)}\end{array}$ & $\left.\begin{array}{c}\text { Heat Generation Rate: 100MW } \\
\text { Fr in }\end{array}\right],\left(\frac{B T U}{m^{3}}\right)$ \\
\hline Fuel & {$[97.0],(168)$} & {$\left[1.82 \times 10^{7} / \mathrm{t}\right],\left(3.26 \times 10^{11} / \mathrm{t}\right)$} \\
\hline Segregation & {$[27.1],(46.9)$} & {$\left[1.87 \times 10^{8} / \mathrm{t}\right],\left(3.34 \times 10^{12} / \mathrm{t}\right)$} \\
\hline Blister & {$[97.0],(168)$} & {$\left[1.51 \times 10^{4} / \mathrm{t}\right],\left(2.70 \times 10^{8} / \mathrm{t}\right)$} \\
\hline
\end{tabular}

Figure 2-3. Axisymmetric Geometry used for Fuel Defect Simulations [13] [17]. The $t$ mils measurement indicated for the thickness of the clad plus filler domain is used as an adjustment factor to vary the thickness of this region, i.e. 10 mils $\leq \boldsymbol{t} \leq \mathbf{5 0 m i l s}$. This thickness adjustment factor is also used to adjust the heat generation rate for the fuel and segregation domains as indicated in the table. 
$\bar{u}=1.0+\left(\alpha_{\text {seg }} \alpha_{\text {blister }}-1.0\right) \frac{h_{h s}}{15,000\left[\frac{B t u}{h r f t^{2 \circ} \mathrm{F}}\right]}$

Here $\alpha_{\text {seg }}$ is the heat flux peaking factor associated with the fuel segregation defect, $\alpha_{b l i s t e r}$ is the heat flux peaking factor associated with the blister defect, $h_{h s}$ is the film coefficient at the location of the defect, and the numerical value of $15,000\left[\frac{B t u}{h r f t^{2 \circ} \mathrm{F}}\right]$ is the average value of the film coefficient used in the defect simulations of Hilvety \& Chapman [13] and Kirkpatrick [17]. The hotspot uncertainty factor is used as a factor of safety in the thermal-hydraulic safety analysis of the HFIR system. It is important to determine a conservative value for this quantity regarding LEU fuel. It will be discussed later how this is to be done.

These defects have the potential to raise the local clad surface temperature in excess of the saturation temperature of the fluid thus resulting in a potential boiling event. The formation of vapor bubbles in the coolant flow channels could result in flow starvation, i.e. lack of sufficient flow to cool the fuel plates. This in turn would result in further increased clad surface temperatures which would eventually lead to a burnout event and failure of the fuel plate.

The author was unable to find material in the literature regarding thermal hydraulic studies of fuel defects caused by the fuel manufacturing process. Several articles exist regarding the formation of defects during irradiation. Pastore, et al. [19] discuss physics based modeling of fission gas, i.e. Xenon and Krypton, swelling and release in $\mathrm{UO}_{2}$ fuel during irradiation using the TRANSURANUS fuel rod analysis code. This work is focused on the material grain-face bubble formation and the subsequent swelling and release on the local hydrostatic stress. Boulore, Struzik, and Gaudier [20] discuss the uncertainty and sensitivity analysis of thermal behavior of nuclear fuel during irradiation. This analysis is carried out using the fuel rod simulation code METEOR V2. Boulore, et al. [20] identify the thermal conductivity of the $\mathrm{UO}_{2}$ fuel, the radial distribution of power in the fuel pellet, local linear heat rate in the fuel rod, and geometry of the pellet as the most sensitive sources of uncertainty. The uncertainty in these 
parameters is modeled using inverse methods. Boulore, et al. state that measurements on irradiated fuel samples show that "the irradiation temperature has an effect on the accumulation of irradiation defects thus impacting the thermal conductivity of the fuel material." Khvostov, Mikityuk, and Zimmermann [21] develop the model GRSW-A to analyze gaseous swelling and fission gas release in $\mathrm{UO}_{2}$ during irradiation. The model that Khvostov, et al. developed is comprehensive and incorporates other models published in the open literature. Locke [22] discusses the behavior of defective fuel, however, the defects that are discussed are mechanical breaks in the cladding material which release fission gas.

\subsection{Review of the Advanced Neutron Source Reactor Thermal Hydraulic Test Loop}

Experimental data from the Advanced Neutron Source Reactor (ANSR) Thermal Hydraulic Test Loop (THTL) is used herein as part of the validation effort. The THTL was an experimental facility used to determine safety limits for the ANSR associated with flow excursion events, i.e. Ledinegg flow instabilities, and burnout events [23] [24]. The ANSR THTL presents a unique experimental data set that has similar characteristics to that of the flow channels in the HFIR core, i.e. high surface heat flux and a long narrow coolant channel. The aspect ratio of the THTL coolant channel, length to thickness, is 399 , which is only $16.9 \%$ less than that of the HFIR. Flow velocities through the THTL range from $62.34 \mathrm{fps}(19.00 \mathrm{~m} / \mathrm{s})$ to $88.58 \mathrm{fps}(27.00 \mathrm{~m} / \mathrm{s})$ which are also comparable to, albeit larger than, the flow velocity characteristic of the HFIR which, as previously stated, is approximately $52.00 \mathrm{fps}(15.85 \mathrm{~m} / \mathrm{s})$ at steady state operation. The average and peak heat fluxes produced in the THTL are stated to be $6 \mathrm{MW} / \mathrm{m}^{2}$ and 12 $\mathrm{MW} / \mathrm{m}^{2}$, respectively [23] [24]. The average heat flux through the HFIR core is calculated to be 2.13 MW/m². Therefore, with higher flow velocities and higher average heat fluxes, the THTL provides extreme cases, relative to HFIR conditions, with which to aid in the validation of COMSOL. 


\subsection{Motivation and Objectives for This Dissertation}

The legacy codes used to determine the thermal hydraulic characteristics of the HFIR fuel plate provide sufficient information that has supported the safe operation of the device for over 50 years. However, the codes are based on assumptions that can be relaxed or even eliminated which provides a more physical representation of the systems or system components that they describe. This is the motivation behind the work presented in this dissertation.

The objectives of this work are to show that COMSOL can accurately represent the system being modeled and provide useful information regarding the thermal hydraulic characteristics of fuel defects in support of the conversion from HEU to LEU fuel. These objectives are met in Chapters 5 where the models of the Advanced Neutron Source Reactor Thermal Hydraulic Test Loop are compared with experimental data, and Chapter 6 where fuel defect models are created and analyzed.

This work is unique in that it provides a more physical representation of the HFIR fuel plate, relative to past work, by modeling the interior of the fuel plate, the turbulent flow field around the fuel plate, and variable fluid properties. 


\section{Chapter 3 Simulation Physics}

It is well known in physics that certain quantities are conserved during the evolution of a system from its initial state to its final state, i.e. mass (if the system is not undergoing nuclear reactions), energy, linear and angular momentum, and charge. In this work we will not be considering the transmutation of nuclei that occurs during the fission process and the resultant loss of neutrons to the environment. Instead we will consider thermal energy generation within the fuel domain which results from the fission process. Therefore, in this work, mass will be a conserved quantity. Further, the interaction of gamma radiation with the coolant and clad materials will not be considered in this work either.

The flow physics found herein will be developed to accommodate changes in coolant material properties, i.e. density, viscosity, isobaric specific heat capacity, and thermal conductivity, with respect to the thermodynamic state. Since the mass density of the fluid can fluctuate due to the large heat fluxes encountered in the reactor core, i.e. $10^{6} \mathrm{~W} / \mathrm{m}^{2}$, compressibility effects must be taken into account [25].

\subsection{Conservation of Mass}

The principle of mass conservation states that for a fixed volume of material the positive time rate of change of the mass of a system is equal to the net inward flux of mass crossing the system boundaries plus sources of mass. As stated previously, sources of mass will not be considered in this work. The principle of mass conservation has the following form.

$\partial_{t} \int \rho(T, P) d V=-\oiint \rho(T, P) \boldsymbol{u}(\boldsymbol{r}, t) \cdot \boldsymbol{n} d A$

Here, for the arbitrary quantity $\alpha, \partial_{\alpha}:=\frac{\partial}{\partial \alpha} \cdot \rho(T, P)$ is the mass density as a function of the thermodynamic state of the system, $d V$ is an infinitesimal volume element of the system, $\boldsymbol{u}(\boldsymbol{r}, t)$ is the flow velocity of the system as a function of position, $\boldsymbol{r}$, and time, $t . \boldsymbol{n}$ is the unit outward normal vector associated with the infinitesimal element of surface area $d A$ bounding the volume 
$d V$. It is important to note that the mass flux, $\rho \boldsymbol{u}$, is integrated over the entire surface that encloses the material volume.

Transposing the time differential and volume integral operators along with imposing the divergence theorem, i.e. $\oiint \boldsymbol{u} \cdot \boldsymbol{n} d A=\int \boldsymbol{\nabla} \cdot \boldsymbol{u} d V$, one arrives at the following relationship.

$$
\int\left[\partial_{t} \rho+\nabla \cdot(\rho \boldsymbol{u})\right] d V=0
$$

Since the volume of the system is entirely arbitrary, one can argue that it is the integrand which vanishes, therefore yielding the general differential form of the mass conservation principle.

$\partial_{t} \rho+\nabla \cdot(\rho \boldsymbol{u})=0$

As previously demonstrated, the flow is fully turbulent. The details of the instantaneous quantities, i.e. random, chaotic fluctuations in density, velocity, and pressure, are interesting and should be investigated from the standpoint of gaining insight into the physical aspects of turbulent flow. This is an active area of research known as Direct Numerical Simulation where all scales of the turbulent flow field are represented. This field of study, however, at this time, cannot model engineering systems as the computational requirements for such a feat far outweigh the capabilities of the largest computers available. As a result, the practicing engineer relies on average flow quantities to make decisions regarding design and performance of systems where turbulence is present.

Osborne Reynolds [25] decomposed the turbulent quantities into average and fluctuating components. For example, the turbulent velocity field is decomposed thusly,

$u_{i}=U_{i}+u_{i}^{\prime}$

Here, $u_{i}$ is the $\mathrm{i}^{\text {th }}$ component of the instantaneous velocity field, $U_{i}$ is the temporal average of the $\mathrm{i}^{\text {th }}$ component of the velocity, and $u_{i}^{\prime}$ is the fluctuation in the $\mathrm{i}^{\text {th }}$ component of the velocity. It is convenient to use tensor notation in the subsequent development of these equations.

The temporal average of an instantaneous flow quantity is found from the evaluation of the following functional. 
$U_{i}=\lim _{\tau \rightarrow \infty} \frac{1}{\tau} \int_{t}^{t+\tau} u_{i} d t$

Here $\tau$ is the observation time. Based on the assumption that in the limit of long observation times, the fluctuating quantities spend equal amounts of time greater than and less than the average, one can assume that

$\bar{u}_{i}^{\prime}=\lim _{\tau \rightarrow \infty} \frac{1}{\tau} \int_{t}^{t+\tau}\left(u_{i}-U_{i}\right) d t=0$.

Equation 3.6 is completely consistent with Equation 3.5. Please be aware that these relationships are not limited to the flow velocity, but apply to any flow quantity with a fluctuating component. Since we are considering compressibility effects it is convenient to consider also the Favre average, or mass average, of the velocity. The Favre averaging procedure is strictly a mathematical convenience [25] and does not change the physical meaning of the equations. The Favre average is computed by the following functional.

$\tilde{u}_{i}=\frac{1}{\bar{\rho}} \lim _{\tau \rightarrow \infty} \frac{1}{\tau} \int_{t}^{t+\tau} \rho(\boldsymbol{r}, t) u_{i} d t$

Here $\bar{\rho}$ is the temporally averaged mass density. Notice that the mass density is a function of position and time. In the cases to be analyzed in this work, the spatial and temporal functionality of the mass density, and any coolant material properties for that matter, will be embedded in the spatial and temporal change of the thermodynamic state of the system, e.g. $\rho(\boldsymbol{r}, t)=$ $\rho[T(\boldsymbol{r}, t), P(\boldsymbol{r}, t)]$. A useful consequence of Equation 3.7 is [25]

$\bar{\rho} \tilde{u}_{i}=\overline{\rho u}_{i}=\overline{\left(\bar{\rho}+\rho^{\prime}\right)\left(U_{l}+u_{\imath}^{\prime}\right)}=\bar{\rho} U_{i}+\overline{\rho^{\prime} u_{l}^{\prime}}$

the average of the flow momentum density. The decomposition of the instantaneous velocity with respect to the Favre procedure is

$u_{i}=\tilde{u}_{i}+u_{i}^{\prime \prime}$ 
The double primed quantity has different characteristics than the Reynolds fluctuation, i.e. single primed fluctuation. First calculate the temporal average of $u_{i}^{\prime \prime}$ from Equation 3.9 then multiply through by the temporally averaged mass density.

$\bar{\rho} \overline{u_{\imath}^{\prime \prime}}=\bar{\rho} \bar{u}_{i}+\bar{\rho} \tilde{u}_{i}$

Substituting in Equations 3.5 and 3.8 and dividing by the temporally averaged mass density yields the relationship of interest.

$\overline{u_{l}^{\prime \prime}}=-\frac{\overline{\rho^{\prime} u_{l}^{\prime}}}{\bar{\rho}}$

While Equation 3.6 states that the long time average of a single fluctuating quantity vanishes, one cannot make the same statement a priori regarding the product of two or more fluctuating quantities due to the potential for these quantities to be statistically dependent, i.e. the fluctuating quantities are potentially correlated thus making their average non-zero. Mathematically, two random variables are correlated if

$\overline{\phi \eta}=\iint_{-\infty}^{\infty} \phi \eta P(\phi, \eta) d \phi d \eta \neq\left[\int_{-\infty}^{\infty} \phi P(\phi) d \phi\right]\left[\int_{-\infty}^{\infty} \eta P(\eta) d \eta\right]=\bar{\phi} \bar{\eta}$

Here $P(\phi, \eta)$ is the joint probability distribution of the variables. A special case of this occurs if $P(\phi, \eta)=P(\phi) P(\eta)$, which leads to the right hand side of Equation 3.12. If the joint probability distribution is equal to the product of the individual probability distributions then the variables are said to be statistically independent or uncorrelated.

A simple physical argument demonstrates that the mass density and velocity fluctuations are indeed correlated, similar arguments regarding the correlation of velocity fluctuations can be found in Tennekes and Lumley [26]. Consider a flow field bound by a heated wall. The increase in fluid temperature due to the thermal energy flux is such that variations in the fluid density occur. Now consider such a density fluctuation manifested as a local decrease in mass density at the point $\boldsymbol{r}$, i.e. $-\rho^{\prime}(\boldsymbol{r})$. This fluctuation affects the fluid in the neighborhood, $\boldsymbol{r}+\boldsymbol{\epsilon}$, with $\boldsymbol{\epsilon}>0$, of the point $\boldsymbol{r}$ by pushing fluid away, therefore creating a fluctuation in the velocity field. Since the density fluctuation created the velocity fluctuation in this scenario, the correlation between 
these quantities does not vanish. Indeed, the ability to conceive of a physical situation in which quantities are correlated without knowledge to the contrary requires one to maintain that the correlation does not vanish.

The mass conservation equation for compressible turbulent flow is written as

$\partial_{t}\left[\left(\bar{\rho}+\rho^{\prime}\right)\right]+\partial_{x_{j}}\left[\left(\bar{\rho}+\rho^{\prime}\right)\left(\tilde{u}_{i}+u_{i}^{\prime \prime}\right)\right]=0$

Distributing the quantities in the brackets gives

$\partial_{t} \bar{\rho}+\partial_{t} \rho^{\prime}+\partial_{x_{j}}\left[\bar{\rho} \tilde{u}_{i}+\bar{\rho} u_{i}^{\prime \prime}+\rho^{\prime} \tilde{u}_{i}+\rho^{\prime} u_{i}^{\prime \prime}\right]=0$

Taking the temporal average of Equation 3.14 yields the Favre averaged turbulent mass conservation equation.

$\partial_{t} \bar{\rho}+\partial_{x_{j}}\left[\bar{\rho} \tilde{u}_{i}\right]=0$

\subsection{Conservation of Momentum}

The conservation of momentum principle states that for a fixed volume of material the time rate of change of momentum within the volume is equal to the net influx of momentum plus the influence of surface forces and body forces on the material within the volume. This is written as

$\int \partial_{t}(\rho \boldsymbol{u}) d V=\oiint[-\rho \boldsymbol{u} \boldsymbol{u}+\overline{\overline{\boldsymbol{\sigma}}}] \cdot \boldsymbol{n} d A+\int \boldsymbol{F} \rho d V$

$\partial_{t}(\rho \boldsymbol{u})$ is the local time derivative of the fluid momentum field per unit volume. The second term, $\rho \boldsymbol{u} \boldsymbol{u}$, is the momentum flux and the third term is the second order tensor stress field, $\overline{\overline{\boldsymbol{\sigma}}}$, and $\boldsymbol{F}$ is the body force per unit mass acting on the material volume. The momentum flux may need a bit more clarification. The juxtaposition of the velocity vectors is a dyadic product, i.e. $\boldsymbol{u} \boldsymbol{u}=u_{j} u_{i}$, which is a second order tensor with the same dimensionality of the stress field. Therefore, the sum of the velocity dyad and the stress field is justified. It is implied that the mass density in Equation 3.16 is a function of the thermodynamic state of the system and that the 
velocity field is a function of position and time. Using the divergence theorem, Equation 3.16 has the following form.

$$
\int\left[\partial_{t}(\rho \boldsymbol{u})+\boldsymbol{\nabla} \cdot(\rho \boldsymbol{u} \boldsymbol{u})-\boldsymbol{\nabla} \cdot \overline{\overline{\boldsymbol{\sigma}}}-\boldsymbol{F}\right] d V=\mathbf{0}
$$

As stated before, since the volume of the system is arbitrary, it is the integrand that vanishes. Therefore, the differential relationship for the fluid momentum is

$\partial_{t}(\rho \boldsymbol{u})+\boldsymbol{\nabla} \cdot(\rho \boldsymbol{u u})-\boldsymbol{\nabla} \cdot \overline{\overline{\boldsymbol{\sigma}}}-\boldsymbol{F}=\mathbf{0}$.

The stress field, $\overline{\overline{\boldsymbol{\sigma}}}$, is assumed to have the following constitutive relationship [27]

$\overline{\overline{\boldsymbol{\sigma}}}=-P \overline{\overline{\boldsymbol{I}}}+\mu(P, T)\left[\boldsymbol{\nabla} \boldsymbol{u}+(\boldsymbol{\nabla} \boldsymbol{u})^{T}\right]-\frac{2}{3} \mu(\boldsymbol{\nabla} \cdot \boldsymbol{u}) \overline{\overline{\boldsymbol{I}}}$

Here $P$ is the pressure, $\overline{\bar{I}}$ is the unit tensor, $\mu$ is the dynamic viscosity of the fluid evaluated at the thermodynamic state, $\boldsymbol{\nabla} \boldsymbol{u}$ is the dyadic product of the vector derivative operator and the velocity field which yields a second order tensor, i.e. $\partial_{x_{j}} u_{i},(\nabla \boldsymbol{u})^{T}$ is the transpose of the aforementioned dyadic product. The sum of the dyadic products in the second term on the right hand side, $\boldsymbol{\nabla u}+(\boldsymbol{\nabla u})^{T}$, constitutes the strain rate tensor. Combining Equations 3.19 and 3.18 gives

$\partial_{t}(\rho \boldsymbol{u})+\boldsymbol{\nabla} \cdot(\rho \boldsymbol{u} \boldsymbol{u})=-\boldsymbol{\nabla} \cdot(P \overline{\overline{\boldsymbol{I}}})+\boldsymbol{\nabla} \cdot\left\{\mu\left[\boldsymbol{\nabla u}+(\boldsymbol{\nabla u})^{T}\right]\right\}-\frac{2}{3} \boldsymbol{\nabla} \cdot[\mu(\boldsymbol{\nabla} \cdot \boldsymbol{u}) \overline{\overline{\boldsymbol{I}}}]+\boldsymbol{F}$.

This is the conservative invariant form of the compressible Navier-Stokes equation set. To ease the subsequent development, tensor notation will be employed again. Rewriting Equation 3.20 in tensor notation gives

$\partial_{t}\left(\rho u_{i}\right)+\partial_{x_{j}}\left(\rho u_{j} u_{i}\right)=-\partial_{x_{j}} P \delta_{i j}+\partial_{x_{j}}\left[\mu\left(\partial_{x_{j}} u_{i}+\partial_{x_{i}} u_{j}\right)\right]-\frac{2}{3} \partial_{x_{j}}\left(\mu \partial_{x_{k}} u_{k}\right) \delta_{i j}+F_{i}$.

Now that the equation of fluid motion has been stated, we can continue with the development of the Favre averaged Navier-Stokes equation. The decomposition of the velocity field is identical to Equation 3.9, i.e. $u_{i}=\tilde{u}_{i}+u_{i}^{\prime \prime}$. The pressure and, again, the density fields 
will be decomposed in the way of Reynolds, i.e. $\rho=\bar{\rho}+\rho^{\prime}$ and $P=\bar{P}+P^{\prime}$. Substituting these turbulent relationships into Equation 3.20a gives

$$
\begin{aligned}
\partial_{t}\left[( \overline { \rho } + \rho ^ { \prime } ) \left(\tilde{u}_{i}\right.\right. & \left.\left.+u_{i}^{\prime \prime}\right)\right]+\partial_{x_{j}}\left[\left(\bar{\rho}+\rho^{\prime}\right)\left(\tilde{u}_{j}+u_{j}^{\prime \prime}\right)\left(\tilde{u}_{i}+u_{i}^{\prime \prime}\right)\right] \\
& =-\partial_{x_{j}}\left(\bar{P}+P^{\prime}\right) \delta_{i j}+\partial_{x_{j}}\left\{\mu\left[\partial_{x_{j}}\left(\tilde{u}_{i}+u_{i}^{\prime \prime}\right)+\partial_{x_{i}}\left(\tilde{u}_{j}+u_{j}^{\prime \prime}\right)\right]\right\} \\
& -\frac{2}{3} \partial_{x_{j}}\left[\mu \partial_{x_{k}}\left(\tilde{u}_{k}+u_{k}^{\prime \prime}\right)\right] \delta_{i j} .
\end{aligned}
$$

Expanding all terms and taking the temporal average of these terms gives

$$
\begin{aligned}
\partial_{t}\left(\bar{\rho} \tilde{u}_{i}\right)+\partial_{x_{j}}( & \left.\bar{\rho} \tilde{u}_{j} \tilde{u}_{i}+\overline{\rho u_{\jmath}^{\prime \prime} u_{l}^{\prime \prime}}\right) \\
= & -\partial_{x_{j}} \bar{P} \delta_{i j}+\partial_{x_{j}}\left[\mu\left(\partial_{x_{j}} \tilde{u}_{i}+\partial_{x_{j}} \overline{u_{l}^{\prime \prime}}+\partial_{x_{i}} \tilde{u}_{j}+\partial_{x_{i}} \overline{u_{\jmath}^{\prime \prime}}\right)\right] \\
& -\frac{2}{3} \partial_{x_{j}}\left[\mu\left(\partial_{x_{k}} \tilde{u}_{k}+\partial_{x_{k}} \overline{u_{k}^{\prime \prime}}\right)\right] .
\end{aligned}
$$

Intuitively one expects that $\partial_{x_{\alpha}} \overline{u_{\beta}^{\prime \prime}} \ll \partial_{x_{\alpha}} \tilde{u}_{\beta}$, it has been stated that this is the case for virtually all flows [28]. This follows from the intuitive argument that each fluctuating quantity is some scalar multiple of the conjugate average quantity, e.g. $u_{i}^{\prime}=\chi U_{i} ;\{\chi: \mid-1<\chi<1\}$ and $\rho^{\prime}=$ $\eta \bar{\rho} ;\{\eta: \mid-1<\eta<1\}$, where the scalar is a random variable. The relative range of the fluctuations will not be as large as those stated. Indeed, the supremum and infimum stated are extreme and the probability of these values being realized is low. However, given our limited knowledge of turbulent phenomena, one must consider the possibility of very large fluctuations and, therefore, the limits on the random variables were chosen as $(-1,1)$. For the sake of progress and to be consistent with the literature, we will discount the possibility of very large fluctuations and assume that the fluctuations are relatively small. One then finds that indeed the Favre fluctuations can be neglected relative to the Favre average, i.e.

$\overline{u_{l}^{\prime \prime}}=-\frac{\overline{\rho^{\prime} u_{l}^{\prime}}}{\bar{\rho}}=-\frac{\overline{\eta \rho \chi U_{l}}}{\bar{\rho}}=-\overline{\eta \chi} U_{i}<U_{i}$

Under the same argument, the Favre average velocity is expressed as 
$\tilde{u}_{i}=U_{i}+\frac{\overline{\rho^{\prime} u_{l}^{\prime}}}{\bar{\rho}}=U_{i}(1+\overline{\eta \chi}) \approx U_{i}$

Using these arguments, one can neglect the $\partial_{x_{\alpha}} \overline{u_{\beta}^{\prime \prime}}$ terms. Thus yielding the Favre averaged Navier-Stokes equations.

$$
\begin{aligned}
\partial_{t}\left(\bar{\rho} \tilde{u}_{i}\right)+\partial_{x_{j}} & \left(\bar{\rho} \tilde{u}_{j} \tilde{u}_{i}\right) \\
& =-\partial_{x_{j}} \bar{P} \delta_{i j}+\partial_{x_{j}}\left[\mu\left(\partial_{x_{j}} \tilde{u}_{i}+\partial_{x_{i}} \tilde{u}_{j}\right)\right]-\frac{2}{3} \partial_{x_{j}}\left[\mu\left(\partial_{x_{k}} \tilde{u}_{k}\right)\right] \\
& -\partial_{x_{j}}\left(\overline{\rho u_{\jmath}^{\prime \prime} u_{\imath}^{\prime \prime}}\right) .
\end{aligned}
$$

This is the final result. The last term on the right hand side of Equation 3.25 is the troublesome Reynolds stress tensor. Assuming that turbulent flows can indeed be represented by the NavierStokes equation set, it is this Reynolds stress tensor that is the root cause of the theoretical problem of turbulence. Notice that if the Reynolds stress tensor vanishes, the NS Equation set

for laminar flow, Equation 3.20a, is recovered. Further note that if the velocity fluctuations were uncorrelated then by Equation 3.6 and Equation 3.12 the Reynolds stress tensor would vanish.

In an effort to write the Reynolds stress in terms of the dynamical variable $\tilde{u}_{i}$, Boussinesq proposed a shear-stress strain relationship [29] which introduces the so-called eddy viscosity. Using the Favre averaged procedure, the Boussinesq hypothesis has the following form [25]

$-\overline{\rho u_{\jmath}^{\prime \prime} u_{\imath}^{\prime \prime}}=\rho v_{t}\left(\partial_{x_{j}} \tilde{u}_{i}+\partial_{x_{i}} \tilde{u}_{j}\right)-\frac{1}{3} \overline{\rho u_{k}^{\prime \prime} u_{k}^{\prime \prime}} \delta_{i j}$

Here $\rho v_{t}=\mu_{t}$ is the so-called eddy viscosity which has been the focus of many modeling efforts. The turbulence closure models used in this work are based on an attempt to model the eddy viscosity.

\subsubsection{The k- $\omega$ Closure Model}

The eddy viscosity is defined in the k- $\omega$ equation set as [25] [30] 
$\mu_{T}=\frac{\bar{\rho} k_{t u r b}}{\widetilde{\omega}}$.

Here $k_{t u r b}$ is the specific turbulent kinetic energy which is defined as

$k_{\text {turb }}:=\frac{1}{2} \frac{\overline{\rho u_{\imath}^{\prime \prime} u_{\jmath}^{\prime \prime}}}{\bar{\rho}} \delta_{i j}$,

and $\widetilde{\omega}$ is the conditional specific dissipation rate of the turbulent kinetic energy which will be defined shortly.

The specific turbulent kinetic energy is modeled by the transport equation [25] [30]

$$
\begin{aligned}
\partial_{t}\left(\bar{\rho} k_{t u r b}\right)+ & \partial_{x_{j}}\left(\bar{\rho} \tilde{u}_{j} k_{t u r b}\right) \\
& =\partial_{x_{j}} \tilde{u}_{i}\left[2 \mu_{T}\left(\partial_{x_{j}} \tilde{u}_{i}+\partial_{x_{i}} \tilde{u}_{j}-\frac{1}{3} \partial_{x_{m}} \tilde{u}_{m} \delta_{i j}\right)-\frac{2}{3} \bar{\rho} k_{t u r b} \delta_{i j}\right] \\
& -\beta^{*} \bar{\rho} k_{t u r b} \omega+\partial_{x_{j}}\left[\left(\mu+\sigma^{*} \frac{\bar{\rho} k_{t u r b}}{\omega}\right) \partial_{x_{j}} k_{t u r b}\right] .
\end{aligned}
$$

Here $\beta^{*}$ and $\sigma^{*}$ are closure coefficients with values of 0.09 and 3/5, respectively [28] [30]. The specific dissipation rate, $\omega$, is determined by the transport equation

$$
\begin{aligned}
\partial_{t} \omega+\partial_{x_{j}}\left(\bar{\rho} \tilde{u}_{j} \omega\right) & \\
= & \alpha \frac{\omega}{k_{t u r b}} \partial_{x_{j}} \tilde{u}_{i}\left[2 \mu_{T}\left(\partial_{x_{j}} \tilde{u}_{i}+\partial_{x_{i}} \tilde{u}_{j}-\frac{1}{3} \partial_{x_{m}} \tilde{u}_{m} \delta_{i j}\right)-\frac{2}{3} \bar{\rho} k_{t u r b} \delta_{i j}\right] \\
& -\beta \bar{\rho} \omega^{2}+\left(\sigma_{d} \frac{\bar{\rho}}{\omega} \partial_{x_{j}} k_{t u r b}\right) \partial_{x_{j}} \omega+\partial_{x_{j}}\left[\left(\mu+\sigma \frac{\bar{\rho} k_{t u r b}}{\omega}\right) \partial_{x_{j}} \omega\right] .
\end{aligned}
$$

Here $\alpha$ and $\sigma$ are closure constants with respective values of $13 / 25$ and $1 / 2$. The coefficient $\beta$ is defined by the following relationship [28] [30] 


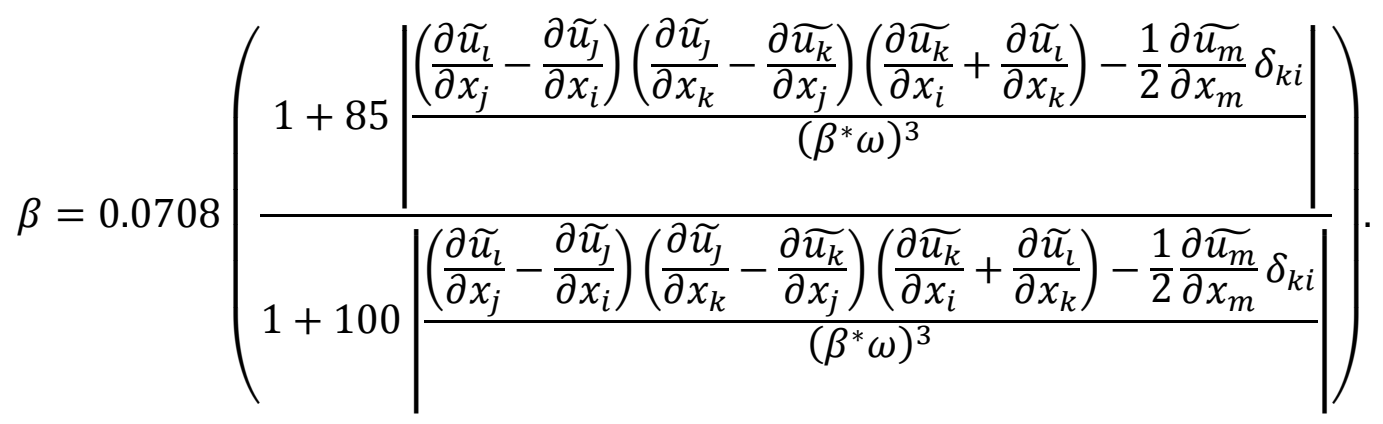

The coefficient $\sigma_{d}$ has the following characteristic [28]

$$
\sigma_{d}=\left\{\begin{array}{c}
0 \text { for } \frac{\partial k_{t u r b}}{\partial x_{j}} \frac{\partial \omega}{\partial x_{j}} \leq 0 \\
\frac{1}{8} \text { for } \frac{\partial k_{t u r b}}{\partial x_{j}} \frac{\partial \omega}{\partial x_{j}}>0
\end{array}\right.
$$

where

$$
\widetilde{\omega}=\max \left\{\omega, \quad \frac{7}{8} \sqrt{\frac{2\left(\frac{\partial \widetilde{u_{l}}}{\partial x_{j}}+\frac{\partial \widetilde{u_{j}}}{\partial x_{i}}-\frac{1}{3} \frac{\partial \widetilde{u_{m}}}{\partial x_{m}} \delta_{i j}\right)^{2}}{\beta^{*}}}\right\}
$$

This concludes the k- $\omega$ turbulence model.

\subsubsection{The k- $\varepsilon$ Closure Model}

The eddy viscosity for the k- $\varepsilon$ model is defined as [25] [28] [30]

$$
\mu_{T}=\bar{\rho} C_{\mu} \frac{k_{\text {turb }}^{2}}{\epsilon} .
$$


The empirically determined closure coefficient, $C_{\mu}$, is equal to 0.09 . The specific turbulent kinetic energy, $k_{t u r b}$, for the k- $\varepsilon$ is modeled by the transport equation [30]

$$
\begin{aligned}
\partial_{t}\left(\bar{\rho} k_{t u r b}\right)+ & \frac{\partial}{\partial x_{j}}\left(\bar{\rho} \tilde{u}_{j} k_{t u r b}\right) \\
& =\partial_{x_{j}} \tilde{u}_{i}\left[2 \mu_{T}\left(\partial_{x_{j}} \tilde{u}_{i}+\partial_{x_{i}} \tilde{u}_{j}-\frac{1}{3} \partial_{x_{m}} \tilde{u}_{m} \delta_{i j}\right)-\frac{2}{3} \bar{\rho} k_{t u r b} \delta_{i j}\right]-\bar{\rho} \epsilon \\
& +\partial_{x_{j}}\left[\left(\mu+\frac{\mu_{T}}{\sigma_{k}}\right) \partial_{x_{j}} k_{t u r b}\right] .
\end{aligned}
$$

The dissipation of turbulent kinetic energy, $\epsilon$, is determined by the transport equation [30]

$$
\begin{aligned}
\partial_{t}(\bar{\rho} \epsilon)+\partial_{x_{j}}\left(\bar{\rho} \tilde{u}_{\jmath} \epsilon\right) & \\
= & C_{\epsilon 1} \frac{\epsilon}{k_{t u r b}} \partial_{x_{j}} \tilde{u}_{i}\left[2 \mu_{T}\left(\partial_{x_{j}} \tilde{u}_{i}+\partial_{x_{i}} \tilde{u}_{j}-\frac{1}{3} \partial_{x_{m}} \tilde{u}_{m} \delta_{i j}\right)\right. \\
& \left.-\frac{2}{3} \bar{\rho} k_{t u r b} \delta_{i j}\right]-C_{\epsilon 2} \bar{\rho} \frac{\epsilon^{2}}{k_{t u r b}}+\partial_{x_{j}}\left[\left(\mu+\frac{\mu_{T}}{\sigma_{\epsilon}}\right) \partial_{x_{j}} \epsilon\right] .
\end{aligned}
$$

Here $\sigma_{k}$ is equal to $1.0, \sigma_{\epsilon}$ is equal to $1.3, C_{\epsilon 1}$ is equal to 1.44 , and $C_{\epsilon 2}$ is equal to 1.92 [28] [30]. These are empirically determined closure coefficients. This concludes the standard k- $\varepsilon$ model.

\subsubsection{The Low Reynolds Number k- $\varepsilon$ Model}

The eddy viscosity for this model has been significantly modified

$$
\mu_{T}=\bar{\rho} C_{\mu} \frac{k_{t u r b}^{2}}{\epsilon}\left\{1-\exp \left[-\frac{\epsilon^{\frac{1}{4}} l_{w}}{14}\left(\frac{\bar{\rho}}{\mu}\right)^{\frac{3}{4}}\right]\right\}^{2}\left[1-5\left(\frac{\mu \epsilon}{\bar{\rho} k_{\text {turb }}^{2}}\right)^{\frac{3}{4}} \exp \left(-\frac{\bar{\rho} k_{\text {turb }}^{2}}{200 \mu \epsilon}\right)^{2}\right]
$$


Here the closure coefficient, $C_{\mu}$, has not changed in value. Here $l_{w}$ is the distance to the closest wall [30]. The closure coefficients have different values which are $C_{\epsilon 1}=1.5, C_{\epsilon 2}=1.9$, and $\sigma_{\epsilon}=$ $\sigma_{k}=1.4$.

The transport equation for the specific turbulent kinetic energy is identical to the standard k- $\varepsilon$ model and, therefore, will not be repeated here. The transport equation for the dissipation of the specific turbulent kinetic energy, $\epsilon$, is modified with a damping coefficient, $f_{\epsilon}[30]$. This equation is given below.

$$
\begin{aligned}
\partial_{t}(\bar{\rho} \epsilon)+\partial_{x_{j}}\left(\bar{\rho} \tilde{u_{j}} \epsilon\right) & \\
= & C_{\epsilon 1} \frac{\epsilon}{k_{t u r b}} \partial_{x_{j}} \tilde{u}_{i}\left[2 \mu_{T}\left(\partial_{x_{j}} \tilde{u}_{i}+\partial_{x_{i}} \tilde{u}_{j}-\frac{1}{3} \partial_{x_{m}} \tilde{u}_{m} \delta_{i j}\right)\right. \\
& \left.-\frac{2}{3} \bar{\rho} k_{t u r b} \delta_{i j}\right]-f_{\epsilon} C_{\epsilon 2} \bar{\rho} \frac{\epsilon^{2}}{k_{t u r b}}+\partial_{x_{j}}\left[\left(\mu+\frac{\mu_{T}}{\sigma_{\epsilon}}\right) \partial_{x_{j}} \epsilon\right] .
\end{aligned}
$$

The damping coefficient is represented by the following relationship

$$
f_{\epsilon}=\left\{1-\exp \left[-\frac{\epsilon^{\frac{1}{4}} l_{w}}{3.1}\left(\frac{\bar{\rho}}{\mu}\right)^{\frac{3}{4}}\right]\right\}^{2}\left[1-0.3 \exp \left(-\frac{\bar{\rho} k_{\text {turb }}^{2}}{6.5 \mu \epsilon}\right)^{2}\right]
$$

This concludes the low Reynolds number turbulence model.

\subsubsection{The Spallart-Allmaras Model}

The Spallart-Allmaras model was considered, however, the author was unable to obtain a solution without imposing inconsistent stabilization, i.e. numerical viscosity. Since this can have a drastically adverse effect on the solution, this model was not considered further and will not be mentioned again. It will be shown that the low Reynolds number $\mathrm{k}-\varepsilon$ model is superior to the 
wall offset models in that it computes variables in the laminar sublayer of the turbulent boundary layer.

\subsubsection{Turbulent Model Closure Discussion}

The effects of using a wall-offset model incorrectly, i.e. not having the grid sufficiently far from the wall, are shown in Figure 3-1. Figure 3-1 is based on a test case which imposes a turbulent flow field over a flat plate. This discussion is necessary due to the default value for $\mathrm{y}^{+}$ within COMSOL being set to a minimum value of 11.06 . The variable $\mathrm{y}^{+}$is a measure of the distance from the wall where the logarithmic layer of the turbulent boundary layer begins. Experimentally, the valid range is $30<\mathrm{y}^{+}<300$ [31] [32]. Here the $\mathrm{k}-\varepsilon$ wall-offset model is compared with the LRN model for different minimum values of $\mathrm{y}^{+}$set for the first element away from the wall. The first element is located a distance of $1.75 \times 10^{-7} \mathrm{~m}$ from the wall. It should be noted that the k- $\omega$ model produces the same results as the k- $\varepsilon$ model. The LRN is used as the basis for this comparison due to the models ability to match the experimentally determined turbulent velocity boundary layer, i.e. to resolve thermal-fluid variable in the viscous sublayer of the turbulent boundary layer. Figure 3-1 shows that forcing a minimum value of $\mathrm{y}^{+}=30$ at a distance of $1.75 \times 10^{-7} \mathrm{~m}$ from the wall over estimates the fluid velocity by a factor of 52.8 ! Similarly, forcing a minimum value of $\mathrm{y}^{+}=1$ at the same distance over estimates the fluid velocity by a factor of 24.4. An over estimate of the fluid velocity produces an over estimate of the shear stress in the fluid at the wall and, more importantly for this case, an over estimate of the convection coefficient. An over estimate of the convection coefficient leads to artificially low surface temperatures. Given the importance associated with the surface temperature distribution for the HFIR, i.e. potential for boiling, it is necessary to predict this distribution as accurately as possible. Therefore, if one is to use the wall-offset models, it is imperative that the grid be appropriately spaced to avoid inaccurate results. 


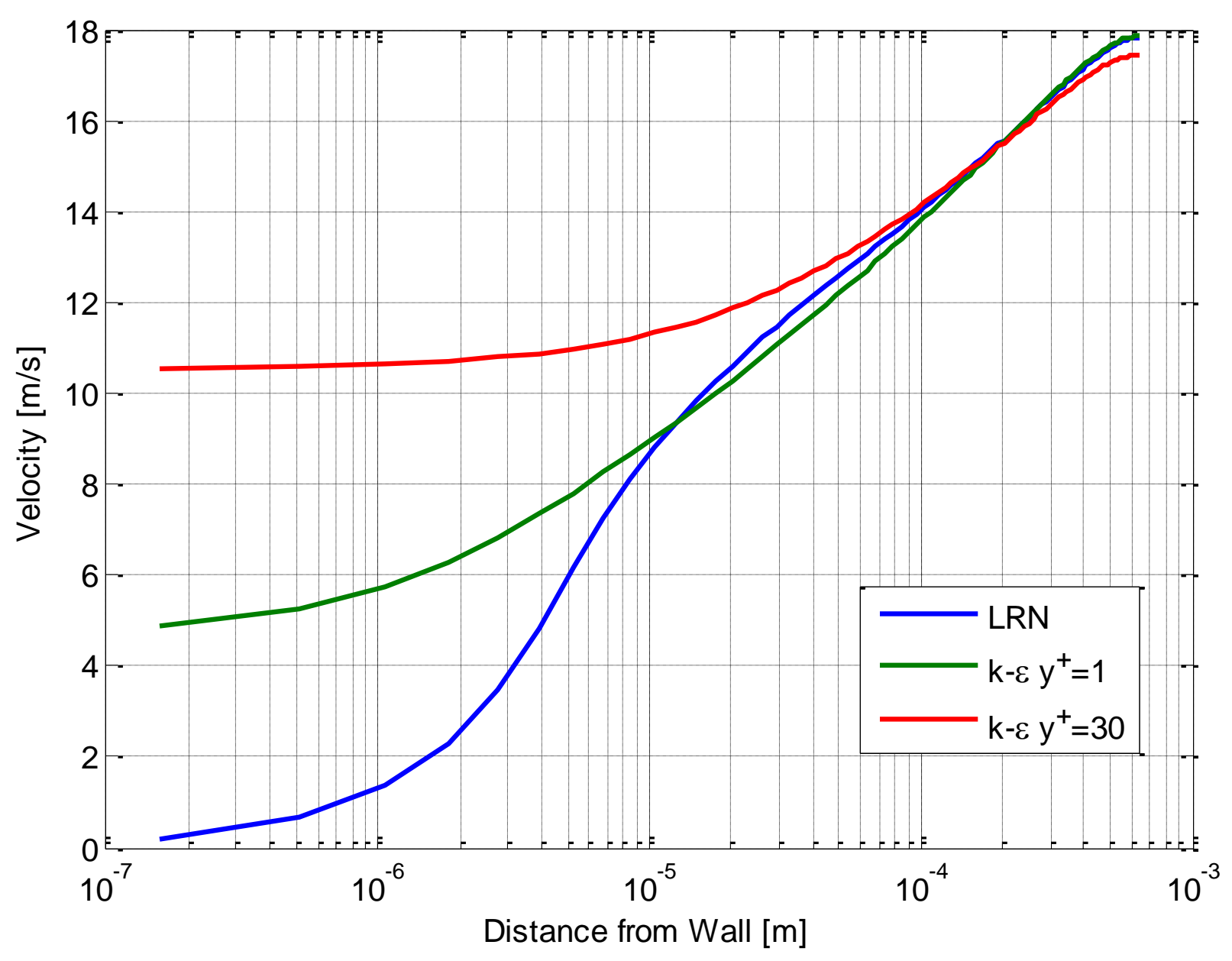

Figure 3-1. The Effect of Wall-Offset Models with Grid Elements Too Close to the Wall. The cut-line used for this plot was created at $\boldsymbol{y}=\mathbf{0 . 3 0 4 7} \mathrm{m}$, i.e. at the exit of the heated section. 


\subsection{Conservation of Energy}

The conservation of energy principle states that the time rate of change of the internal energy content per unit volume of material is equal to the net influx of energy through the surface bounding the volume plus the addition of heat, work done on the system by surface forces, and sources of thermal energy.

$$
\begin{aligned}
\partial_{t} \int\left[\rho\left(e+\frac{1}{2} \boldsymbol{u} \cdot \boldsymbol{u}+\Phi\right)\right] d V \\
\quad=\oiint\left[-\rho\left(e+\frac{1}{2} \boldsymbol{u} \cdot \boldsymbol{u}+\Phi\right) \boldsymbol{u}+\boldsymbol{u} \cdot \overline{\overline{\boldsymbol{\sigma}}}-\boldsymbol{q}^{\prime \prime}\right] \cdot \boldsymbol{n} d A+\int q^{\prime \prime \prime} d V
\end{aligned}
$$

Here $e$ is the specific internal energy of the material volume, $\frac{1}{2} \boldsymbol{u} \cdot \boldsymbol{u}$ is the specific kinetic energy of the material volume, $\Phi$ is the specific potential energy of the material volume, $\boldsymbol{q}^{\prime \prime}$ is the heat flux vector, and $q^{\prime \prime \prime}$ is the thermal energy generation rate per unit volume which can also be described as the power density. Again, transposing the temporal derivative operator and the volume integral, and using the divergence theorem, one arrives at the following equation.

$$
\int\left\{\partial_{t}\left[\rho\left(e+\frac{1}{2} \boldsymbol{u} \cdot \boldsymbol{u}+\Phi\right)\right]+\boldsymbol{\nabla} \cdot\left[\rho \boldsymbol{u}\left(e+\frac{1}{2} \boldsymbol{u} \cdot \boldsymbol{u}+\Phi\right)-\boldsymbol{u} \cdot \overline{\overline{\boldsymbol{\sigma}}}+\boldsymbol{q}^{\prime \prime}\right]-q^{\prime \prime \prime}\right\} d V=0
$$

Since this equation is based upon an arbitrary volume one can assume that the integrand vanishes thus revealing the differential form of the conservation of energy principle.

$\partial_{t}\left[\rho\left(e+\frac{1}{2} \boldsymbol{u} \cdot \boldsymbol{u}+\Phi\right)\right]+\nabla \cdot\left[\rho \boldsymbol{u}\left(e+\frac{1}{2} \boldsymbol{u} \cdot \boldsymbol{u}+\Phi\right)-\boldsymbol{u} \cdot \overline{\overline{\boldsymbol{\sigma}}}+\boldsymbol{q}^{\prime \prime}\right]-q^{\prime \prime \prime}=0$

Substituting Equation 3.19

$\overline{\overline{\boldsymbol{\sigma}}}=-P \overline{\overline{\boldsymbol{I}}}+\mu\left[\boldsymbol{\nabla} \boldsymbol{u}+(\boldsymbol{\nabla u})^{T}\right]-\frac{2}{3} \mu(\boldsymbol{\nabla} \cdot \boldsymbol{u}) \overline{\overline{\boldsymbol{I}}}$

into Equation 3.41 gives the following relationship 


$$
\begin{aligned}
\partial_{t}\left[\rho\left(e+\frac{1}{2} \boldsymbol{u} \cdot \boldsymbol{u}+\Phi\right)\right]+\boldsymbol{\nabla} \cdot\left[\rho \boldsymbol{u}\left(e+\frac{1}{2} \boldsymbol{u} \cdot \boldsymbol{u}+\Phi\right)\right] \\
=-\boldsymbol{\nabla} \cdot \boldsymbol{q}^{\prime \prime}-\boldsymbol{\nabla} \cdot[P \boldsymbol{u} \cdot \overline{\overline{\boldsymbol{I}}}]+\boldsymbol{\nabla} \cdot\left\{\mu \boldsymbol{u} \cdot\left[\boldsymbol{\nabla u}+(\boldsymbol{\nabla u})^{T}\right]\right\} \\
-\frac{2}{3} \boldsymbol{\nabla} \cdot[\mu(\boldsymbol{\nabla} \cdot \boldsymbol{u}) \boldsymbol{u} \cdot \overline{\overline{\boldsymbol{I}}}]+q^{\prime \prime \prime}
\end{aligned}
$$

Equation 3.43 is a general relationship for the conservation of energy principle. Slight modifications to this general relationship make the equation well suited for the conservation of energy of a flow field, namely, the introduction of the enthalpy relationship. Equation 3.43 also contains within it the elements for the conservation of energy for solid materials, i.e. materials with no flow characteristics over short observation times. The systems of interest are not extended in space to a degree where a significant change in potential energy would result. Therefore, in what follows the potential energy term, $\Phi$, will be neglected.

\subsubsection{Energy Conservation Principle Applied to the Flow Field}

It is convenient, in applications to flow field energy conservation, to introduce the enthalpy per unit mass [9] [33],

$H=e+\frac{P}{\rho}$

This quantity is usually designated by a lower case $\mathrm{h}$, however, in this work the lower case $\mathrm{h}$ is set aside for the convection coefficient. Now, solving for the specific internal energy in the specific enthalpy equation and substituting the result into the second term of Equation 3.43 gives

$$
\begin{aligned}
\partial_{t}\left[\rho\left(e+\frac{1}{2} \boldsymbol{u} \cdot \boldsymbol{u}\right)\right]+\boldsymbol{\nabla} \cdot\left[\rho \boldsymbol{u}\left(H+\frac{1}{2} \boldsymbol{u} \cdot \boldsymbol{u}\right)\right] \\
=\boldsymbol{\nabla} \cdot(\boldsymbol{u} P)-\boldsymbol{\nabla} \cdot \boldsymbol{q}^{\prime \prime}-\boldsymbol{\nabla} \cdot[P \boldsymbol{u} \cdot \overline{\overline{\boldsymbol{I}}}]+\boldsymbol{\nabla} \cdot\left\{\mu \boldsymbol{u} \cdot\left[\boldsymbol{\nabla u}+(\boldsymbol{\nabla u})^{T}\right]\right\} \\
-\frac{2}{3} \boldsymbol{\nabla} \cdot[\mu(\boldsymbol{\nabla} \cdot \boldsymbol{u}) \boldsymbol{u} \cdot \overline{\overline{\boldsymbol{I}}}]+q^{\prime \prime \prime} .
\end{aligned}
$$


The second and fourth terms on the right hand side cancel, i.e. $\boldsymbol{\nabla} \cdot[P \boldsymbol{u} \cdot \overline{\bar{I}}]=\boldsymbol{\nabla} \cdot(\boldsymbol{u} P)$. Therefore, the final relationship for the conservation of energy principle applied to the flow field is

$$
\begin{aligned}
\partial_{t}\left[\rho\left(e+\frac{1}{2} \boldsymbol{u} \cdot \boldsymbol{u}\right)\right]+\boldsymbol{\nabla} \cdot\left[\rho \boldsymbol{u}\left(H+\frac{1}{2} \boldsymbol{u} \cdot \boldsymbol{u}\right)\right] \\
=-\boldsymbol{\nabla} \cdot \boldsymbol{q}^{\prime \prime}+\boldsymbol{\nabla} \cdot\left\{\mu \boldsymbol{u} \cdot\left[\boldsymbol{\nabla u}+(\boldsymbol{\nabla u})^{T}\right]\right\}-\frac{2}{3} \boldsymbol{\nabla} \cdot[\mu(\boldsymbol{\nabla} \cdot \boldsymbol{u}) \boldsymbol{u} \cdot \overline{\overline{\boldsymbol{I}}}]+q^{\prime \prime \prime}
\end{aligned}
$$

From classical, i.e. equilibrium, thermodynamics the enthalpy is related to the temperature through the relationship,

$d H=C_{p} d T$

Here $C_{p}$ is the isobaric specific heat capacity. Integrating relationship 3.47 yields

$H-H_{0}=\int_{T_{0}}^{T} C_{p} d T$.

Here $H_{0}$ is the enthalpy at the reference temperature, $T_{0}$. In cases where the isobaric specific heat capacity is constant or varies negligibly from $T_{0}$ to $T$, the specific enthalpy can be simply written as

$H=C_{p} T$

since $H_{0}=C_{p} T_{0}$ in this case. Also if the isometric specific heat capacity is constant then the internal energy of the system can be written as $e=C_{v} T$, i.e. the product of the isometric specific heat capacity and the temperature. For flow systems with negligible variance in either specific heat capacity over the observed temperatures, the conservation of energy principle is written as

$$
\begin{aligned}
C_{v} \partial_{t}(\rho T)+\partial_{t} & \left(\rho \frac{1}{2} \boldsymbol{u} \cdot \boldsymbol{u}\right)+C_{p} \boldsymbol{\nabla} \cdot(\rho \boldsymbol{u} T)+\boldsymbol{\nabla} \cdot\left[\rho \boldsymbol{u}\left(\frac{1}{2} \boldsymbol{u} \cdot \boldsymbol{u}\right)\right] \\
& =\partial_{t} P-\boldsymbol{\nabla} \cdot \boldsymbol{q}^{\prime \prime}+\boldsymbol{\nabla} \cdot\left\{\mu \boldsymbol{u} \cdot\left[\boldsymbol{\nabla u}+(\boldsymbol{\nabla u})^{T}\right]\right\}-\frac{2}{3} \boldsymbol{\nabla} \cdot[\mu(\boldsymbol{\nabla} \cdot \boldsymbol{u}) \boldsymbol{u} \cdot \overline{\overline{\boldsymbol{I}}}]+q^{\prime \prime \prime}
\end{aligned}
$$


In flow systems where the variation of the isobaric specific heat capacity cannot be neglected, Equation 3.46 can be written in terms of the dynamic variable, $T$, by making the assumption that both the isobaric and isometric specific heat capacities can be written as a power series in the temperature variable where the coefficients are used to fit the curve with measured data, i.e.

$C_{p}(T)=\psi_{i} T^{i} \quad$ for $\{i: \mid i=0,1,2 \cdots\}$

$C_{v}(T)=\beta_{j} T^{j} \quad$ for $\{j: \mid j=0,1,2 \cdots\}$

and therefore, the specific enthalpy and the specific internal energy is written as

$H=\frac{\psi_{i}}{i+1}\left(T^{i+1}-T_{0}^{i+1}\right)+H_{0}$

$e=\frac{\beta_{j}}{j+1}\left(T^{j+1}-T_{0}^{j+1}\right)+e_{0}$

The indices used are to indicate that the isobaric and isometric specific heat capacities are in general different functions, therefore, different coefficients are used with different degrees of interpolation. If these relationships are substituted into Equation 3.46, then the conservation of energy principle is rendered nonlinear. This is a terrible prospect since the severity of the nonlinearity is directly related to the number of terms which are necessary to adequately interpolate the specific heat data.

One avenue of investigation pursued by the author for steady flows uses arguments similar to those found regarding the definition of the bulk fluid temperature and the way in which it is handled in the literature. The bulk fluid temperature is defined as

$\dot{m} C_{p} T_{b}=\int H d \dot{m}$

The relationship for the specific enthalpy is assumed to be that of Equation 3.49 [9], therefore, Equation 3.53 takes the form 
$\dot{m} C_{p} T_{b}=\int \rho u C_{p} T d A_{c}$

Where $A_{c}$ is the cross sectional area normal to the flow direction. Equation 3.54 is solved for $T_{b}$ with the result

$T_{b}=\frac{1}{\dot{m} C_{p}} \int \rho u C_{p} T d A_{c}$

In general, the velocity, $u$, and the fluid temperature, $T$, are distributed along the cross-section. Now the statement is made by Incropera [9] that, "if the density and specific heat are constant, then these quantities come outside the integral". This implies that if the specific heat capacity is not constant then it must remain under the integral operation. This statement is not consistent with classical thermodynamics since it has already been assumed, in progressing from Equation 3.53 to Equation 3.54 that the specific enthalpy is written as in Equation 3.49, i.e. $H=C_{p} T$. From the standpoint of classical thermodynamics, writing Equation 3.54 immediately implies that the specific heats cancel.

However, if we consider writing the specific enthalpy as $H=C_{p} T$, and allow for variation of the specific heat capacity, then we can consider temporal and spatial variations in the specific heat capacity within the energy conservation principle. Writing the specific enthalpy this way has been done in the literature [34] [35]. Now the conservation of energy principle is

$$
\begin{aligned}
\boldsymbol{\nabla} \cdot\left[\rho \boldsymbol{u} C_{p} T+\right. & \left.\rho \boldsymbol{u} \frac{1}{2}(\boldsymbol{u} \cdot \boldsymbol{u})\right] \\
& =-\boldsymbol{\nabla} \cdot \boldsymbol{q}^{\prime \prime}+\boldsymbol{\nabla} \cdot\left\{\mu \boldsymbol{u} \cdot\left[\boldsymbol{\nabla u}+(\boldsymbol{\nabla u})^{T}\right]\right\}-\frac{2}{3} \boldsymbol{\nabla} \cdot[\mu(\boldsymbol{\nabla} \cdot \boldsymbol{u}) \boldsymbol{u} \cdot \overline{\overline{\boldsymbol{I}}}]+q^{\prime \prime \prime}
\end{aligned}
$$

As stated earlier, the specific heat capacity is a function of the thermodynamic state which in turn is a spatio-temporal function, i.e. $C_{p}=C_{p}[T(\boldsymbol{r}, t), P(\boldsymbol{r}, t)]$. As a result, the derivatives of the specific heat capacity, that occur on the left hand side of Equation 3.56 are

$\boldsymbol{\nabla} C_{p}=\frac{\partial C_{p}}{\partial T} \nabla T+\frac{\partial C_{p}}{\partial P} \nabla P$ 
This too renders the conservation of energy principle, as shown in Equation 3.46, nonlinear, however, the nonlinearity is not as extreme as in the case associated with Equation 3.52. To show this we expand the terms on the left hand side of Equation 3.56.

$$
\begin{aligned}
\rho C_{p}[\boldsymbol{u} \cdot \boldsymbol{\nabla} T]+ & \rho T\left[\frac{\partial C_{p}}{\partial T}(\boldsymbol{u} \cdot \boldsymbol{\nabla} T)+\frac{\partial C_{p}}{\partial P}(\boldsymbol{u} \cdot \boldsymbol{\nabla} P)\right]+\boldsymbol{\nabla} \cdot\left[\rho \boldsymbol{u} \frac{1}{2}(\boldsymbol{u} \cdot \boldsymbol{u})\right] \\
& =-\boldsymbol{\nabla} \cdot \boldsymbol{q}^{\prime \prime}+\boldsymbol{\nabla} \cdot\left\{\mu \boldsymbol{u} \cdot\left[\boldsymbol{\nabla} \boldsymbol{u}+(\boldsymbol{\nabla u})^{T}\right]\right\}-\frac{2}{3} \boldsymbol{\nabla} \cdot[\mu(\boldsymbol{\nabla} \cdot \boldsymbol{u}) \boldsymbol{u} \cdot \overline{\overline{\boldsymbol{I}}}]+q^{\prime \prime \prime}
\end{aligned}
$$

The nonlinearity in Equation 3.57 is

$\rho T\left[\frac{\partial C_{p}}{\partial T}(\boldsymbol{u} \cdot \nabla T)\right]$

While this nonlinearity does add difficulty, it is not as difficult or uncertain as having a nonlinearity of $O\left(T^{i+2}\right)$. The results associated with the use of the turbulent version of Equation 3.57 will be discussed with regard to the Advanced Neutron Source Reactor Thermal Hydraulic Test Loop in that chapter.

The Favre averaging process will be applied to the energy principle for flow systems. Using the arguments and relationships found in both the conservation of mass and conservation of momentum developments, and incorporating the Favre averaged specific enthalpy and heat flux, i.e. $H=\widetilde{H}+H^{\prime \prime}$ and $q^{\prime \prime}=\widetilde{q^{\prime \prime}}+\left(q^{\prime \prime}\right)^{\prime \prime}$ where $\left(q^{\prime \prime}\right)^{\prime \prime}$ is the Favre heat flux fluctuation, the Favre averaged flow energy equation is written in tensor notation as

$$
\begin{aligned}
\partial_{t}\left(\bar{\rho} \tilde{e}+\frac{1}{2} \bar{\rho} \tilde{u}_{i} \tilde{u}_{i}+k_{\text {turb }}\right)+\partial_{x_{j}}\left[\bar{\rho} \tilde{u}_{j}\left(\widetilde{H}+\frac{1}{2} \tilde{u}_{i} \tilde{u}_{i}+\frac{k_{t u r b}}{\bar{\rho}}\right)\right] \\
=-\partial_{x_{j}} \widetilde{q_{j}^{\prime \prime}}+\partial_{x_{j}}\left[\mu \tilde{u}_{j}\left(\partial_{x_{j}} \tilde{u}_{i}+\partial_{x_{i}} \tilde{u}_{j}\right)\right]-\frac{2}{3} \partial_{x_{j}}\left[\mu \tilde{u}_{j}\left(\partial_{x_{k}} \tilde{u}_{k}\right) \delta_{i j}\right]+q^{\prime \prime \prime} .
\end{aligned}
$$

If the specific enthalpy can be written as it is in Equation 3.49, i.e. $H=C_{p} T$, then the Favre averaged heat flux can be written in terms of the Favre averaged specific enthalpy.

$$
\widetilde{q^{\prime \prime}}=-\frac{\mu}{P r} \partial_{x_{j}} \widetilde{H}
$$


Here $\operatorname{Pr}$ is the Prandtl number, i.e. $\operatorname{Pr}=\mu C_{p} /{ }_{k}$. This is the approach used when $C_{p} \neq C_{p}(T)$.

However, when $C_{p}$ is a function of temperature, the averaged heat flux will be written in terms of the temperature, i.e.

$\widetilde{q^{\prime \prime}}=-k_{f} \partial_{x_{j}} \widetilde{T}$

Equation 3.58 with the addition of either Equation 3.59 or Equation 3.60, depending on the case, represents the conservation of energy principle for the turbulent flow field. To be complete, the conservation of energy principle for the flow field with the Fourier law of heat conduction substituted in for the heat flux is

$$
\begin{aligned}
\partial_{t}\left(\bar{\rho} \tilde{e}+\frac{1}{2} \bar{\rho} \tilde{u}_{i} \tilde{u}_{i}+k_{t u r b}\right)+\partial_{x_{j}}\left[\bar{\rho} \tilde{u}_{j}\left(\widetilde{H}+\frac{1}{2} \tilde{u}_{i} \tilde{u}_{i}+\frac{k_{t u r b}}{\bar{\rho}}\right)\right] \\
=\partial_{x_{j}}\left(k_{f} \partial_{x_{j}} \tilde{T}\right)+\partial_{x_{j}}\left[\mu \tilde{u}_{j}\left(\partial_{x_{j}} \tilde{u}_{i}+\partial_{x_{i}} \tilde{u}_{j}\right)\right]-\frac{2}{3} \partial_{x_{j}}\left[\mu \tilde{u}_{j}\left(\partial_{x_{k}} \tilde{u}_{k}\right) \delta_{i j}\right] \\
+q^{\prime \prime \prime} .
\end{aligned}
$$

This is a general form of the equation where the specific internal energy and the specific enthalpy can be written in terms of the temperature, however, the form of these relationships depends on the number of necessary interpolation constants to fit the specific heat data.

\subsubsection{Energy Conservation Principle Applied to Solids}

Solid bodies, by definition, can withstand shear stresses up to the yield stress of the material. The material of the solid body in this work is Al6061 which has a yield stress of $276 \mathrm{MPa}$ [36]. The stresses generated by the turbulent flow field will not be this great, therefore, the cladding will be modeled as a solid. The conservation of energy principle for an arbitrary stationary solid body with negligible potential energy rise is

$\partial_{t}(\rho e)=-\nabla \cdot \boldsymbol{q}^{\prime \prime}+q^{\prime \prime \prime}$ 
Equation 3.62 is a pure transient conduction problem. The equation is linear as long as the material properties of the solid body are, or can be considered to be, constant. Again, using the Fourier law of heat conduction, the transient conduction problem has the form

$\partial_{t}(\rho e)=\partial_{x_{j}}\left(k_{f} \partial_{x_{j}} T\right)+q^{\prime \prime \prime}$.

As was stated earlier, the specific internal energy of the solid can be written as a function of the temperature, however, the form of the relationship is dependent on the number of coefficients necessary to interpolate the specific heat data. 


\section{Chapter 4 2-D Model Development: Turbulent Conjugate Heat Transfer (TCHT)}

The model to be discussed couples the 2-D non-isothermal turbulent flow field with the diffusion of thermal energy within a solid domain. 2-D modeling was performed to show, in a relatively simple way, the influence of variable fluid properties and the influence of distributed thermal energy generation rates on the heat transfer characteristics of the flow field. This portion of the work considers four different situations. First, a constant thermal generation rate is considered with one model employing constant fluid properties and another model using variable fluid properties. Secondly, a distributed thermal generation rate is considered again with two independent models employing constant fluid properties and variable fluid properties.

The plane of interest is that produced by the axial direction and the thickness of the union of one-half of a fuel plate and one-half of a coolant flow channel, refer to Figures 1-1 and 1-2, i.e. the lateral extent is neglected. The model considers the diffusion of thermal information in the axial direction, however lateral thermal diffusion is inherently prohibited by the dimensionality of the model geometry. Since only two dimensions are represented, the physical processes that would occur in the unrepresented dimension cannot be accounted for in the computational domain, e.g. thermal diffusion, transversal fluid flow, and vortex stretching in this instance. The model results were developed using COMSOL version 4.3a.

\subsection{2-D TCHT Model Geometry and Computational Grid Structure}

The simulation geometry for the 2-D TCHT model includes one-half of an inner element fuel plate and one-half of a coolant channel as shown in Figure 4-1. The fuel plate geometry includes both an active fuel domain and a non-active clad domain. The leading edge of the clad domain has a radius of curvature of $0.025 \mathrm{in} .\left(0.635 \times 10^{-4} \mathrm{~m}\right)$, which is consistent with the physical fuel plate of the HFIR. The fluid domain has been extended in both the upstream and downstream directions. The entrance extension in the upstream direction is $0.0267 \mathrm{~m}$ from the leading edge of 
the fuel plate. This extension is necessary to allow the flow to adjust to the obstruction presented by the fuel plate. The exit extension in the downstream direction is $0.2539 \mathrm{~m}$ from the trailing edge of the fuel plate. This extension is necessary to allow the flow to expand and relax the transverse pressure gradient downstream of the fuel plate. This allows one to impose a uniform pressure boundary condition at the global flow outlet of the model.

The inlet boundary condition is set to represent a uniform flow velocity of $7.925 \mathrm{~m} / \mathrm{s}$ and a uniform temperature of $327 \mathrm{~K}$. Since the model inlet has a larger cross sectional area, the inlet velocity was adjusted to ensure that the nominal flow rate through the core was preserved. The left and right global boundaries are symmetric with respect to flow and heat flux, i.e. the flow velocity vector and the heat flux vector normal to these boundaries are set equal to zero. As stated previously, the global exit boundary is set to a uniform pressure of $377.7 \mathrm{psi}(2.606 \mathrm{MPa})$ which is consistent with the exit pressure of the core system [3]. The global exit boundary is set to a convective outlet for the heat transfer condition. The boundary at the interface between the fuel meat and clad is set with a thermal continuity condition. The boundary at the clad-fluid interface is set with a thermal continuity condition and a no-slip velocity condition. The thermal continuity condition implies that the temperature and heat flux are at least piecewise continuous across the boundary.

The details of the computational grid are associated with the numerically labeled regions of Figure 4-2. The high aspect ratio of the computational domain prohibits direct visualization of the grid structure. As a result, the grid structure of the different regions indicated in Figure 4-2 will be discussed in detail so that this structure can be reproduced. It is important to note that there exists a buffer region between region 2 and region 3 that is not indicated in Figure 4-2. This buffer region was necessary to consistently connect the grid structure in region 2 with the grid structure in region 3. This issue will be discussed shortly. It should be mentioned that the grid structure described below is the product of a significant optimization effort which entailed much trial and error on the part of the author. 


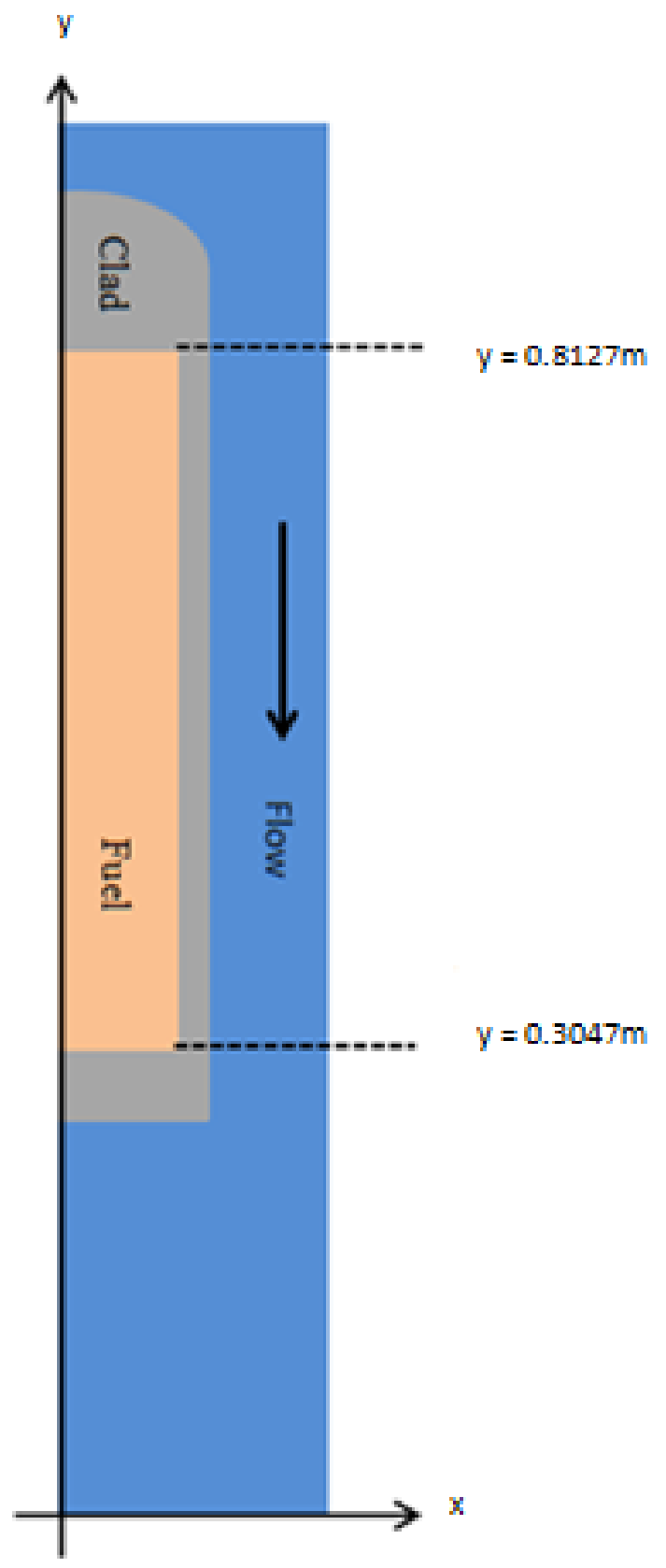

Figure 4-1. 2-D Turbulent Conjugate Heat Transfer Model Geometry. The terminal locations of the active portion of the fuel domain are shown for reference. The flow direction is indicated for reference as well. In order to simplify the analysis, $\boldsymbol{z}=\mathbf{0 . 8 1 2 7} \boldsymbol{m}-\boldsymbol{y}$ will be used as the flow direction variable. 


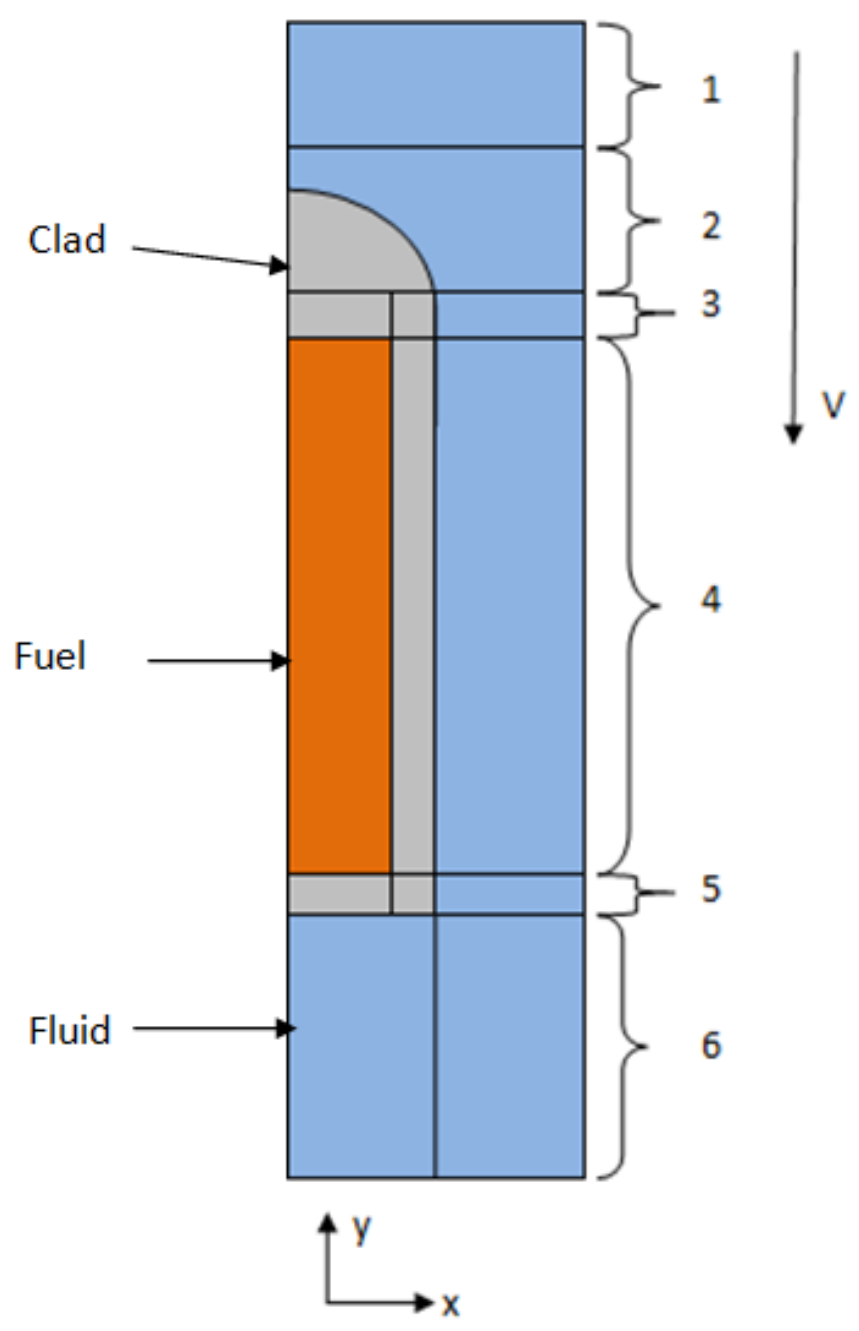

Figure 4-2. 2-D Turbulent Conjugate Heat Transfer Model Geometry. The blue domain is the fluid, the grey domain is the clad, and the orange domain is the fuel meat. The boundaries within each individual domain are artificial and used to develop the computational grid. This is not a scale representation of the computational domain. The dimensions have been greatly distorted for visualization purposes since the aspect ratio, axial length/thickness, $y / x$, is 480 . 
Region 1, of Figure 4-2, is discretized using a highly structured quadrilateral mesh, i.e. mapped mesh, with 10 evenly distributed elements in the x-direction and 8 elements in the $y$ direction distributed with an element ratio of 35 . The element ratio prescribes the ratio in size between the last element in a region and the first element in that same region [30]. Therefore, as the element ratio increases the element size decreases from one boundary to the other, e.g. in this instance the last element, i.e. the element closest to the plate within region 1 of Figure 4-2, will be $1 / 35$ the size of the first element at the global inlet. This feature is used in region 1 in anticipation of upstream deviations in the flow field due to the presence of the fuel plate.

The grid in region 2, of Figure 4-2, is a composite structure consisting of quadrilateral elements layered along the solid boundary in the fluid domain and triangular elements filling in the remainder of the region, i.e. both clad and fluid domains. The triangular grid structure was constructed first to establish the boundary discretization. Then the layered quadrilateral elements, i.e. boundary layer elements, were introduced onto this base structure to capture the development of the boundary layer along the fuel plate. The region 2 grid structure is shown in Figure 4-3.

The region1-region2 interface has 10 elements which is consistent with the region 1 distribution. The right fluid boundary of region 2 has 40 evenly distributed elements, and the left fluid boundary of region 2 has 20 evenly distributed elements. The higher element density on the left fluid boundary was constructed to provide better resolution for the upstream flow disturbances that would be present due to the fuel plate.

The curved leading edge of the fuel plate, i.e. solid-fluid interface, is discretized with 100 elements. Subsequently, 30 layered quadrilateral elements, i.e. boundary layer elements, are placed along the solid-fluid interface with a stretching factor of 1.1. The stretching factor determines the thickness of each quadrilateral element relative to the previous adjacent element, the succession being in a direction normal to the surface along which the elements are placed [30]. Therefore, a stretching factor of 1.1 adjusts the thickness of elements by $10 \%$ relative to the previous element in a direction normal to the solid boundary extending into the fluid domain. 


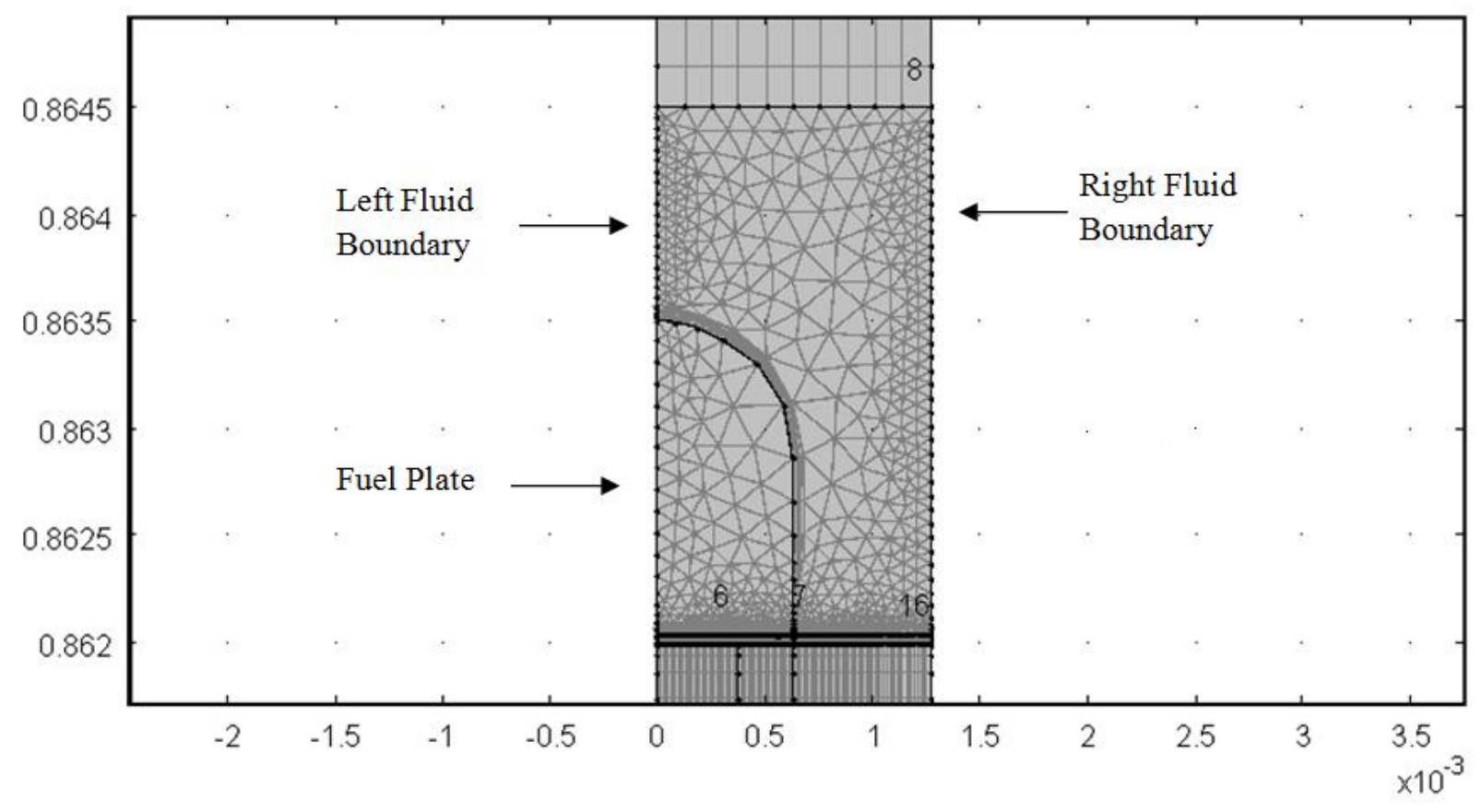

Figure 4-3. Grid Structure of Region 2. The scale represented here has not been modified. 
The thickness of the first layer was set to the default value, which is $1 / 20$ of the local domain element height as stated in the COMSOL documentation [30].

There are 80 triangular elements distributed with an element ratio of 15 along the fluid exit boundary of region 2, i.e. region 2-buffer region interface in the fluid domain, which decrease in size as one approaches the fuel plate. This characteristic of the region 2 grid structure was necessary to remain consistent with the larger mapped structures that exist downstream.

The triangular element structure on the interior of the plate's leading edge does not need to be as dense as the fluid grid structure due to the relatively simple physics to be modeled in that region, i.e. conduction heat transfer. Due to the shared leading edge boundary, the element structure along this boundary is the same as that mentioned previously. The symmetry boundary of the plate in region 2 has 20 elements. The interface region between region 2 and the buffer region, however, has a more specific structure due to the downstream mapped grid restrictions. This boundary has 100 evenly distributed elements.

The buffer region contains only triangular elements. The buffer region was constructed due to difficulties matching the different mesh structures in regions 2 and 3. The element distribution in the $\mathrm{x}$ direction of the buffer region is dictated by the region 2 and region 3 boundaries. The element distribution in the y direction is governed by the solid-fluid interfacial boundary in the buffer region. This boundary has 40 evenly distributed elements.

Region 3 uses a highly structured quadrilateral grid with high element density at the entrance of the region from the buffer region which decreases into a significantly lower element density at the exit passing into region 4 . Region 3 represents $97 \%$ or 1.94 inches $\left(4.93 \times 10^{-2} \mathrm{~m}\right)$ of the upstream 2 inch $\left(5.08 \times 10^{-2} \mathrm{~m}\right)$ unfueled clad section of the fuel plate and coolant channel.

The solid portion of region 3, i.e. the two leftmost artificial domains, has 50 evenly distributed elements in the $\mathrm{x}$ direction per domain. The y direction has 50 elements with an element ratio of 355 with element size decreasing in the upstream direction. The distribution in the y direction was chosen to create a smooth transition in the mesh structure from the high 
element density regions to the reduced element density region of the fueled portion of the fuel plate. Due to the necessity of matching edge discretizations, the fluid domain in this region shares the same distribution in the y direction.

Due to developing velocity boundary layer and thermal boundary layer in region 4 , the mesh structure in the fluid domain of region 3 is dense at the clad-coolant interface and becomes less dense at the right global boundary of the geometry. The boundary discretization in the $\mathrm{x}$ direction in the fluid domain has 80 elements with an element ratio of 15 with size decreasing toward the clad-coolant interface.

The length of the fueled section of the plate is $20 \mathrm{in} .\left(5.08 \times 10^{-1} \mathrm{~m}\right)$. The fueled section is represented by region 4 in Figure 4-2. While slight amounts of heat are deposited in the coolant upstream of this region, most of the heat transfer from the nuclear fuel to the coolant occurs in region 4.

Since the dominant portion of the flow field is in the axial direction, the element density of the flow domain in the axial direction could potentially be significantly reduced. However, there exists a lower limit on the element density which is dictated by the power density profile. The power density profile in the fuel is described, in the SSHTC, by 27 evenly distributed axial points. The minimum axial element density to obtain proper power density resolution is 1.35 elements per inch or 27 evenly distributed axial elements in region 4. Region 5 has a similar element density. In region 5, the element density is rounded up to the nearest integer density value.

The element densities in region 4 and 5 were iterated by integer multiples of this base density to achieve a grid independent solution. The details of this element density parametric sweep will be discussed at length later.

Region 6 again employs the highly structured mapped mesh. The element density at the trailing edge of the fuel plate is very high due to the need to resolve the strong vortex that resides there. This vortex contributes to the relatively high temperatures that are observed at the trailing edge of the fuel plate through the recirculation of the warm fluid. 
The distribution in the $\mathrm{x}$ direction is dictated by the shared boundary between region 5 and region 6 and thus has the same distribution as region 4 in that direction. The axial distribution is set to 270 elements with an element ratio of 2000. As the flow redistributes itself after expansion past the fuel plate, it settles and the effects of the plate on the momentum of the fluid are no longer observed. Thus the element density decreases downstream from the plate.

The same grid structure is used in all models, i.e. 563,730 elements. The iterative convergence is set to $1 \times 10^{-6}$.

\subsection{2-D Turbulent Conjugate Heat Transfer (TCHT) Analysis: Constant Heat} Flux case with Constant and Variable Fluid Properties.

The discussion regarding the constant heat flux model will use the same model geometry shown in Figure 4-1.

The 2-D TCHT analysis uses the Low Reynold's number k- $\varepsilon$ (LRN) turbulence model coupled with thermal diffusion in both the solid and fluid domain. As previously stated, the LRN model allows for the evaluation of thermo-fluid variables through the laminar sublayer of the turbulent boundary layer.

Both the constant fluid property (CFP) case and the NIST fluid property (NFP) case will be discussed. The CFP were evaluated at the average bulk fluid temperature, $\overline{T_{b}}=347.58 \mathrm{~K}$, i.e. the sum of the inlet and outlet bulk fluid temperature divided by 2, from the NIST Steam Tables [8] and are listed in Table 4-1. The thermal conductivity of the clad, $k_{c l a d}$, was set to a value of $167 \mathrm{~W} / \mathrm{m} / \mathrm{K}$. The thermal conductivity of the fuel, $k_{f u e l}$, was set to a value of $132.06 \mathrm{~W} / \mathrm{m} / \mathrm{K}$ as previously stated. 
Table 4-1. Constant Fluid Properties Evaluated at the Average Bulk Fluid Temperature, $\overline{\boldsymbol{T}_{\boldsymbol{b}}}=$ $347.58 \mathrm{~K}$

\begin{tabular}{|c|c|}
\hline Property & Value \\
\hline Thermal Conductivity, $k\left(\overline{T_{b}}\right)$ & $0.6678 \mathrm{~W} / \mathrm{m} / \mathrm{K}$ \\
\hline Isobaric Specific Heat, $C_{p}\left(\overline{T_{b}}\right)$ & $4186.6 \mathrm{~J} / \mathrm{kg} / \mathrm{K}$ \\
\hline Dynamic Viscosity, $\mu\left(\overline{T_{b}}\right)$ & $3.8133 \times 10^{-4} \mathrm{~Pa}^{*} \mathrm{~s}$ \\
\hline Mass Density, $\rho\left(\overline{T_{b}}\right)$ & $976.54 \mathrm{~kg} / \mathrm{m}^{3}$ \\
\hline
\end{tabular}

The NFP were determined from the NIST Steam Tables and established as look up tables in the COMSOL environment over the temperature range $320.00 \mathrm{~K}<\mathrm{T}<394.26 \mathrm{~K}$, and over the pressure range $2.5 \mathrm{MPa}<\mathrm{P}<3.5 \mathrm{MPa}$. The carpet plots of these variable properties can be found in Appendix A. These ranges encompass the standard operational range of the HFIR. It is important to note that the fluid properties, i.e. mass density, isobaric specific heat capacity, viscosity, and thermal conductivity, are relatively insensitive to pressure variation over the aforementioned range. For completeness, the variation of the mass density, isobaric specific heat capacity, thermal conductivity, and molecular viscosity with respect to the pressure was found to be of order $10^{-7}, 10^{-6}$, and $10^{-10}$, respectively. The property variation with respect to temperature, however, is much more pronounced. The variation of the mass density and the isobaric specific heat capacity with respect to the temperature is of order 1 . The variation in the thermal conductivity and the molecular viscosity with respect to temperature is of order $10^{-4}$ and $10^{-3}$, respectively. Therefore, the fluid properties are assumed to be a function of the fluid temperature only since a negligible effect is expected from fluid pressure variance.

Theoretical developments show that as the flow becomes thermally fully developed [9], i.e. 


$$
\frac{d}{d z}\left[\frac{T_{s}(z)-T_{f}(x, z)}{T_{s}(z)-T_{b}(z)}\right] \rightarrow 0,
$$

the convection coefficient becomes constant for the constant heat flux constant fluid property (CFP) case using the bulk fluid temperature as the reference temperature in Newton's law of cooling. Here $T_{f}(x, z)$ is the fluid temperature, $T_{s}(z)$ is the clad surface temperature, and $T_{b}(z)$ is the bulk fluid temperature. The flow direction variable, $z$, is defined as

$z \equiv y_{E A R}-y$

here $y_{E A R}$ is the location of the entrance to the active region, i.e. $y_{E A R}=0.8127 \mathrm{~m}$, as shown in Figure 4-1. The introduction of this flow direction variable makes it easier on the reader to understand the subsequent figures.

It is instructive to discuss the calculation of the bulk fluid temperature, $T_{b}$, at this point as it plays a fundamental role in what follows. The bulk fluid temperature is a marginal distribution in the mathematical sense, i.e.

$T_{b}(z) \equiv \frac{1}{\dot{m} \overline{C_{p}}} \int_{x_{s}}^{x_{m p}} \rho\left(T_{f}(x, z)\right) C_{p}\left(T_{f}(x, z)\right) u(x, z) T_{f}(x, z) d x$

Here the limits of the integration procedure are from the heated surface, $x_{s}$, to the flow channel midplane, $x_{m p}$. To use this definition of the bulk fluid temperature to determine its distribution in the flow direction requires one to perform the integration at different locations in the flow direction. In the COMSOL model, this integration was performed at 51 uniformly spaced locations along the flow direction, i.e.

$z_{n+1}=y_{e a r}-(0.508 / 50) n ; n=0 \cdots 50$

Another way to represent the bulk fluid temperature follows from the relationship for the total power supplied to the fluid up to position $\mathrm{z}$. 


$$
\int_{0}^{A_{H T}} q^{\prime \prime}(x, z) d A_{H T}=\dot{m} \overline{C_{p}} \int_{T_{b}(0)}^{T_{b}(z)} d T_{b}
$$

Here $A_{H T}$ is the heat transfer area. Since only a single surface is heating the fluid in the model, the heat transfer area is $\mathrm{L}^{*} \mathrm{z}$, however, the 2-D model assumes a lateral length of unity. Therefore, Equation 4.5 may be written as

$$
\int_{0}^{z} q^{\prime \prime} d z=\dot{m} \overline{C_{p}} \int_{T_{b}(0)}^{T_{b}(z)} d T_{b}
$$

Carrying out the integration in Equation 4.6, for the constant heat flux case, and rearranging the symbols yields the following relationship for the bulk fluid temperature distribution in the flow direction

$T_{b}(z)=\left(\frac{q^{\prime \prime}}{\dot{m} C_{p}}\right) z+T_{b}(0)$.

This relationship is significantly easier to evaluate than that shown in Equation 4.3. For instance, consider the constant heat flux with NIST fluid properties (NFP) case; all quantities in the integrand of Equation 4.3 are variable and therefore must be evaluated at each location, however, in Equation 4.7, only the specific heat capacity of the fluid needs to be evaluated. It will be shown that the two forms of the bulk fluid temperature yield identical results as one would expect. It is important to note that if the heat flux is distributed along the flow direction then one must either be able to represent the heat flux distribution using common mathematical functions or employ a quadrature rule to evaluate the heat flux integral in Equation 4.6. This case of a distributed heat flux will be discussed in section 4.3.

The constant convection coefficient is due to the parallel nature of the affine functions associated with the solid surface temperature and the bulk fluid temperature in the thermally 
fully developed region for the constant heat flux case, i.e. the temperature difference between the clad and the bulk fluid is constant. Using Newton's law of cooling, the definition of the convection coefficient is

$$
h(z):=\frac{q_{s}^{\prime \prime}}{T_{s}(z)-T_{b}(z)}
$$

Here $q_{s}^{\prime \prime}$ is the constant clad surface heat flux and $h(z)$ is the convection coefficient as a function of the flow direction. The derivative of the convection coefficient in the flow direction is calculated to be

$$
\frac{d h}{d z}=\frac{1}{\left[T_{s}(z)-T_{b}(z)\right]} \frac{d q_{s}^{\prime \prime}}{d z}-\frac{q_{s}^{\prime \prime}}{\left[T_{s}(z)-T_{b}(z)\right]^{2}} \frac{d}{d z}\left[T_{s}(z)-T_{b}(z)\right]
$$

However, since a constant heat flux is being considered here, the first term evaluates to zero which leaves

$$
\frac{d h}{d z}=-\frac{q_{s}^{\prime \prime}}{\left[T_{s}(z)-T_{b}(z)\right]^{2}} \frac{d}{d z}\left[T_{s}(z)-T_{b}(z)\right]
$$

Therefore, it is determined that the change in the convection coefficient in the flow direction is inversely proportional to the square of the temperature difference between the clad surface and the bulk fluid, and directly proportional to the change of this temperature difference in the flow direction. Further, the relationship in Equation 4.10 indicates that the convection coefficient is a decreasing function of the flow direction, given that the surface temperature is larger than the bulk fluid temperature, i.e. the slope of the derivative of the convection coefficient is negative. Equation 4.10 further implies that if the temperature difference between the clad surface and bulk fluid is constant then the convection coefficient does not change in the flow direction and as a result remains constant. The temperature difference between the clad-surface and the bulk- 
fluid for both the CFP and NFP case is shown in Figure 4-4 as a function of the hydraulic diameter normalized flow direction. From Figure 4-4, one notices that the flow for the CFP case, i.e. $\left(\mathrm{T}_{\mathrm{s}}-\mathrm{T}_{\mathrm{b}}\right)$-CFP, becomes fully thermally developed at approximately $20 \mathrm{D}_{\mathrm{h}}$. Also from Figure 4-4, one notices that the temperature difference between the clad surface and the bulk fluid for the CFP case is indeed constant over most of the heated region with the exception of the entrance to the heated region where the flow is thermally developing. Therefore, one should expect the convection coefficient based on the bulk fluid temperature to be constant over most of the heated region with the exception of the thermal entry length where the convection coefficient should decrease to its constant value. These characteristics of the convection coefficient for the CFP case, $h:\left(T_{s}-T_{b}\right)-C F P$, are shown in Figure 4-5. The increase in the convection coefficient for the CFP case that occurs at $z / D_{h}=200$ is due to the difference in temperature, i.e. $T_{s}-T_{b u l k}$, decreasing and the fact that the heat flux has not significantly decreased at this location.

For the NIST fluid property (NFP) constant clad surface heat flux case, Figure 4-4 shows that the temperature difference between the clad surface and the bulk fluid is a decreasing function of the flow direction over most of the heated section. This is due to the increase in the thermal conductivity of the fluid with increasing temperature. As the thermal conductivity increases, more thermal energy is passed from the clad surface to the fluid. This has two effects: one, it increases the fluid temperature which further increases the fluid thermal conductivity, and two, the clad surface temperature increase is not as pronounced as it is in the CFP case. As a result, the clad surface temperature increases at a slower rate than the bulk fluid temperature and the representative affine functions are no longer parallel, thus resulting in a decreasing temperature difference between the clad surface and the bulk fluid, as observed in Figure 4-5. Equation 4.10 demonstrates that the convection coefficient should increase due to its dependence on the inverse of the square of the temperature difference between the clad surface and the bulk fluid. This characteristic of the convection coefficient based on NFP is shown in Figure 4-5 as the $h:\left(T_{s}-T_{b}\right)-N F P$ data. 


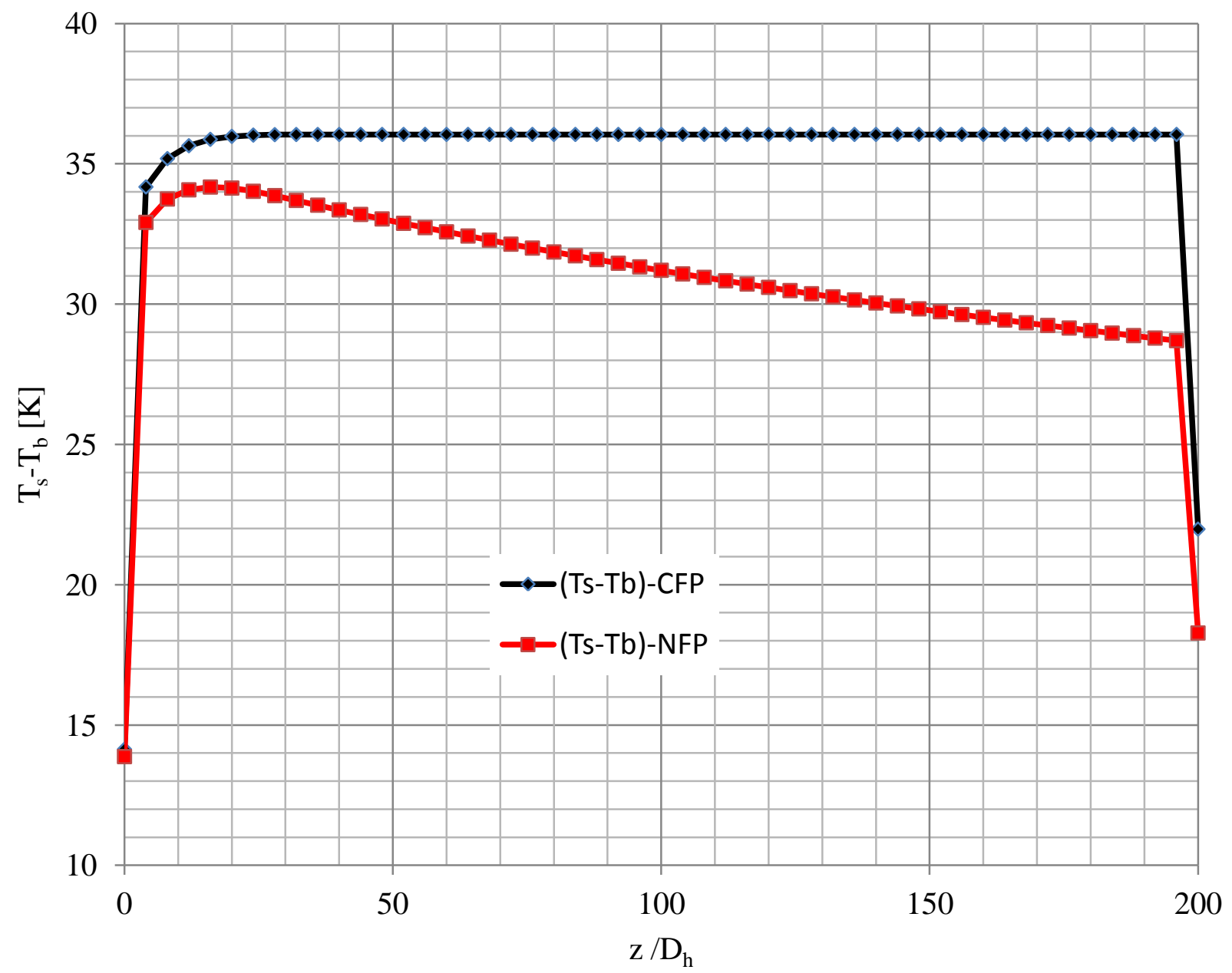

Figure 4-4. Temperature Difference Between the Clad Surface and the Bulk Fluid for Constant Fluid Properties (CFP) and NIST Fluid Properties (NFP). One notices that the system becomes fully thermally developed at approximately $20 \mathrm{D}_{\mathrm{h}}$ for the CFP case. 


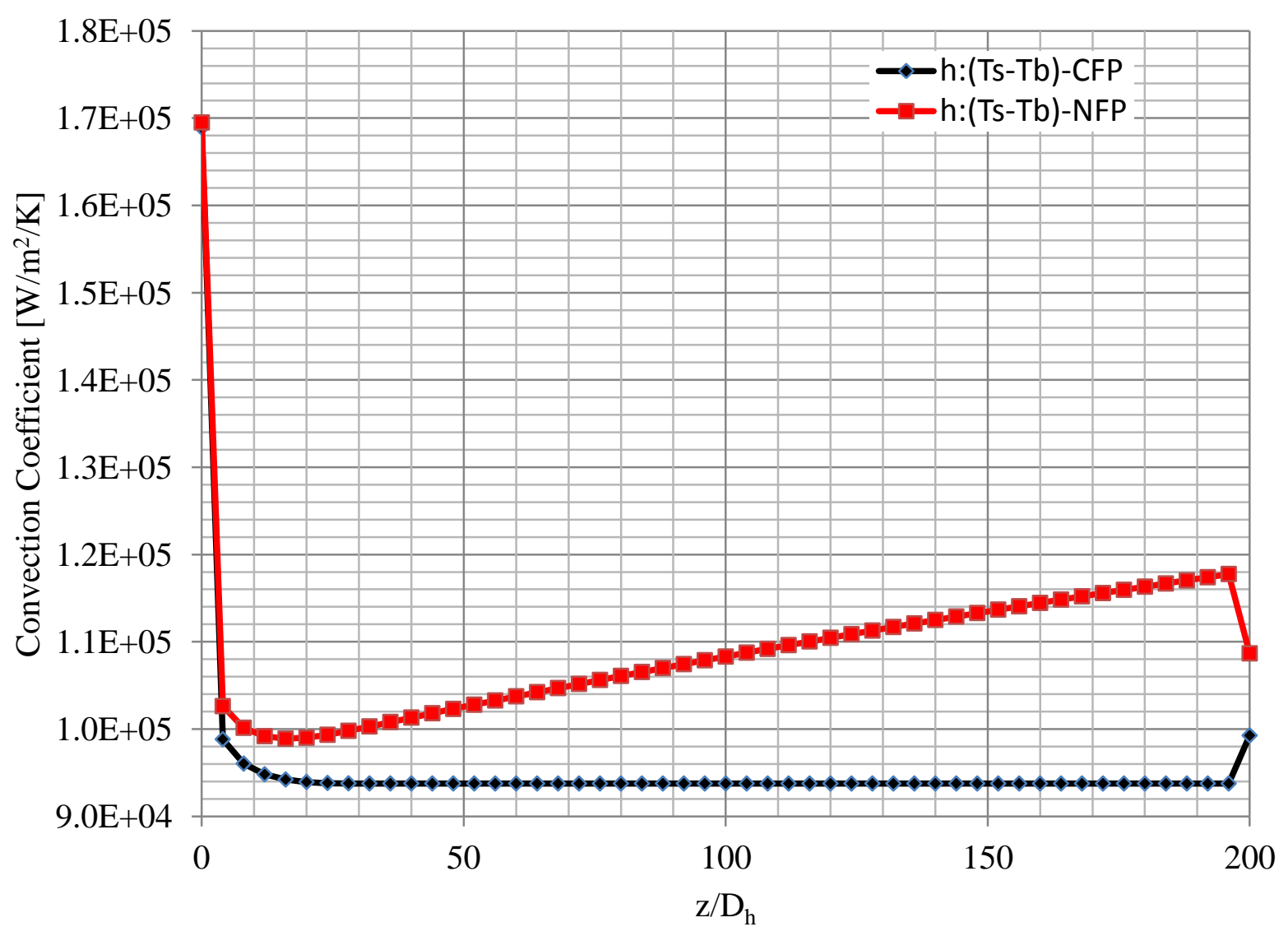

Figure 4-5. Convection Coefficient for Constant Fluid Properties (CFP) and NIST Fluid Properties (NFP). The two outliers occurring at $\mathrm{z}=200 \mathrm{D}_{\mathrm{h}}$ is due to end effects where the end of the active region resides. 
The distribution difference between the cross-sectional fluid temperature and the inlet bulk fluid temperature for the CFP case, $\Theta_{\mathrm{CFP}}=T_{f_{C F P}}(x, z)-T_{i n}$, is shown in Figure 4-6 at several axial locations in the active section of the model. This plot shows the penetration of the thermal information from the heated surface into the flow field. From Figure 4-6, one notices that thermal information from the clad surface does not reach the channel mid-line before $\mathrm{z}=$ $5 D_{\mathrm{h}}$. A similar plot is shown in Figure 4-7 for the NFP case, $\Theta_{\mathrm{NFP}}=T_{f_{N F P}}(x, z)-T_{i n}$. Again, the thermal information from the clad surface reaches the channel mid-line at $\mathrm{z}=5 \mathrm{D}_{\mathrm{h}}$.

The difference between $\Theta_{\mathrm{CFP}}$ and $\Theta_{\mathrm{NFP}}$ is shown in Figure 4-8. This plot translates into a difference between the fluid temperature for the CFP case and the fluid temperature for the NFP case, i.e. $\Theta_{C F P}-\Theta_{N F P}=T_{f_{C F P}}(x, z)-T_{f_{N F P}}(x, z)$. Figure 4-8 provides visual evidence of the differences between Figures 4-6 and 4-7. Figure 4-8 shows that at the wall, i.e. $\mathrm{x}=6.35 \times 10^{-4} \mathrm{~m}$, the CFP fluid temperature is higher than the NFP fluid temperature for all values of $z$ represented. One notices from Figure 4-8 that as one proceeds in the axial direction the value of $\Theta_{C F P}-\Theta_{N F P}$ increases. This is due to the lower thermal conductivity of the fluid in the CFP case relative to the NFP case. Since the fluid thermal conductivity is lower in the CFP case the surface temperature is higher which implies that the fluid adjacent to the wall will also have a higher temperature than the NFP case. However, a short distance away from the wall in the normal direction, the NFP fluid temperature becomes higher and thermal energy penetrates further into the flow field as indicated by the negative values of $\Theta_{C F P}-\Theta_{N F P}$ in conjunction with the extent of this negative region in the flow direction. This is a direct consequence of the increase in the fluid thermal conductivity with increasing temperature, see appendix A, i.e. more thermal energy is allowed to pass from the heated surface to the fluid thus increasing the fluid temperature relative to the $\mathrm{CFP}$ case and as a result increase the thermal conductivity. The curves then again pass to positive values of $\Theta_{C F P}-\Theta_{N F P}$ which indicate that CFP fluid temperature is again higher in magnitude than the NFP fluid temperature. 


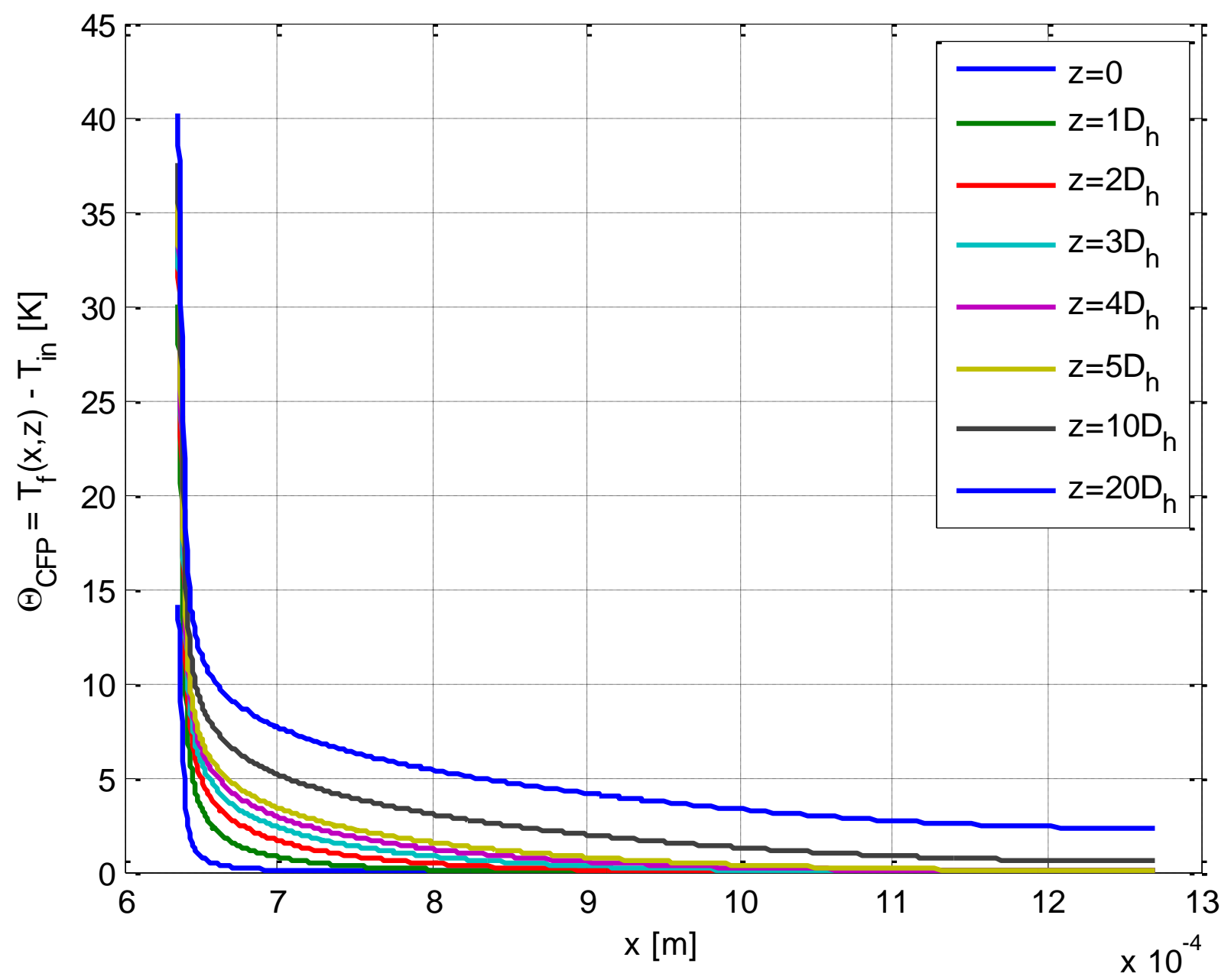

Figure 4-6. Difference between the Fluid Temperature and the Inlet Bulk Fluid Temperature for the Constant Fluid Property Case at Various Locations Along the Heated Section of the Simulation Geometry. The $x$-coordinate begins on the clad surface, i.e. $x=6.35 \times 10^{-4} \mathrm{~m}$, and extends to the fluid mid-channel, i.e. $\mathrm{x}=12.7 \times 10^{-4} \mathrm{~m}$. 


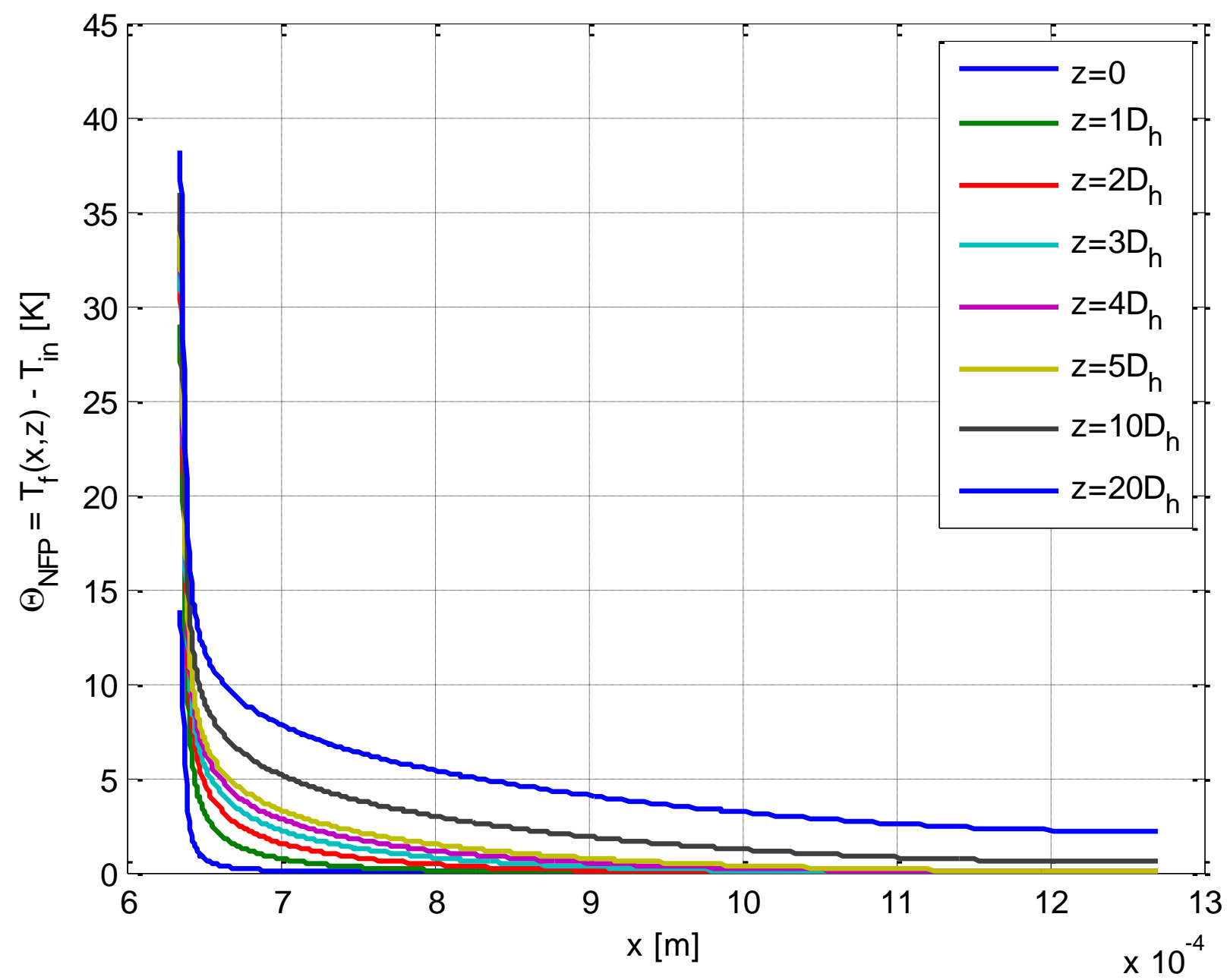

Figure 4-7. Difference between the Fluid Temperature and the Inlet Bulk Fluid Temperature for the Variable Fluid Property Case at Various Locations Along the Heated Section of the Simulation Geometry. The $x$-coordinate begins on the clad surface, i.e. $x=6.35 \times 10^{-4} \mathrm{~m}$, and extends to the fluid mid-channel, i.e. $\mathrm{x}=12.7 \times 10^{-4} \mathrm{~m}$. 


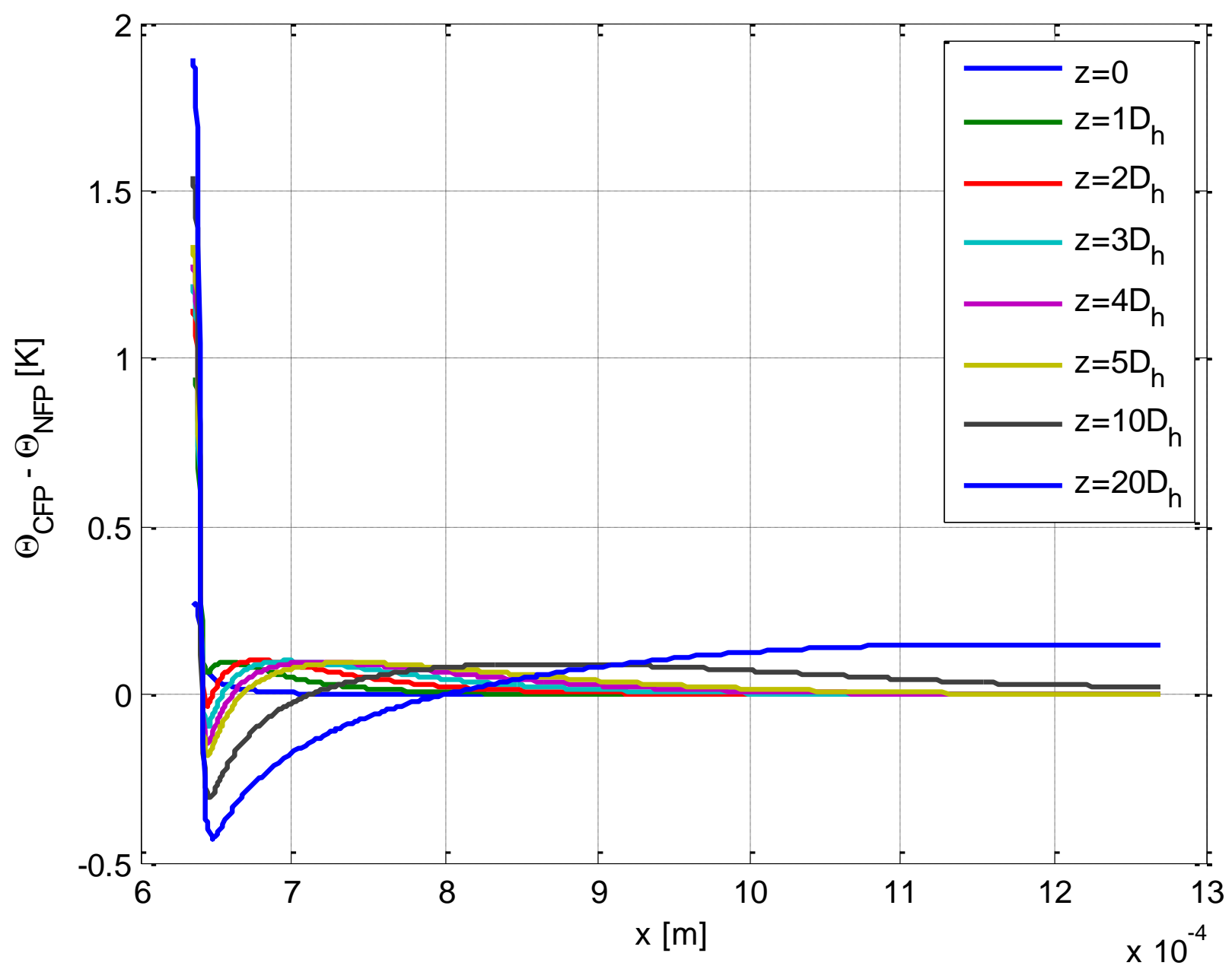

Figure 4-8. Difference between the CFP Fluid Temperature Distribution and the NFP Fluid Temperature Distribution at Different Axial Locations. 
For completeness the clad surface temperatures and the bulk fluid temperatures for both the CFP case and the NFP case are shown in Figure 4-9. This figure shows that it is indeed the surface temperature that is most affected by the use of variable fluid properties, i.e. a clad surface temperature difference of $8 \mathrm{~K}$ is observed at the exit of the active region between the two cases. Further, one observes that the rate of increase in the bulk fluid temperature for the NFP case is negligibly smaller than the CFP case. Therefore, the affine temperature functions, i.e. clad surface temperature and bulk fluid temperature, for the NFP case are indeed not parallel with the difference in the two temperatures decreasing in the flow direction. Further, the average bulk fluid temperature, i.e. $\bar{T}_{b}=347.58 \mathrm{~K}$, is shown to be lower in magnitude than the surface temperature with the exception of the axial location $\mathrm{z}=0$ which was used in the explanation of Figure 4-8. The temperature characteristics shown in Figure 4-9 imply that the use of constant fluid properties for systems with constant heat flux and moderate bulk fluid temperature rises, i.e. $\Delta T_{b} \leq 40 \mathrm{~K}$, produce conservative estimates of the clad surface temperature. Another advantage of the use of constant properties relative to the use of variable properties is the reduction in computational expense. Variable properties are introduced into the computational environment either as look up tables or as analytical functions. During the computational process, the look up tables are interpolated between thermodynamic states or the analytical functions are evaluated, whatever the case may be, for each computational node at each iteration of the computation. A typical grid structure is, at least, of the order of $10^{5}$ nodes with iterations of the order $10^{2}$ for convection problems, therefore the evaluation of variable material properties is highly expensive in the computational sense.

From the convection coefficient, one can form the Nusselt modulus by using the well known relationship

$N u_{D}=\frac{h D_{h}}{k_{f}}$.

For the CFP case, as shown in Figure 4-5, the convection coefficient is constant with a numerical value of $93,748.65 \mathrm{~W} / \mathrm{m}^{2} / \mathrm{K}$. This value of the convection coefficient composed with the thermal conductivity evaluated at the average bulk fluid temperature, i.e. $\mathrm{k}=0.6678 \mathrm{~W} / \mathrm{m} / \mathrm{K}$, and the 


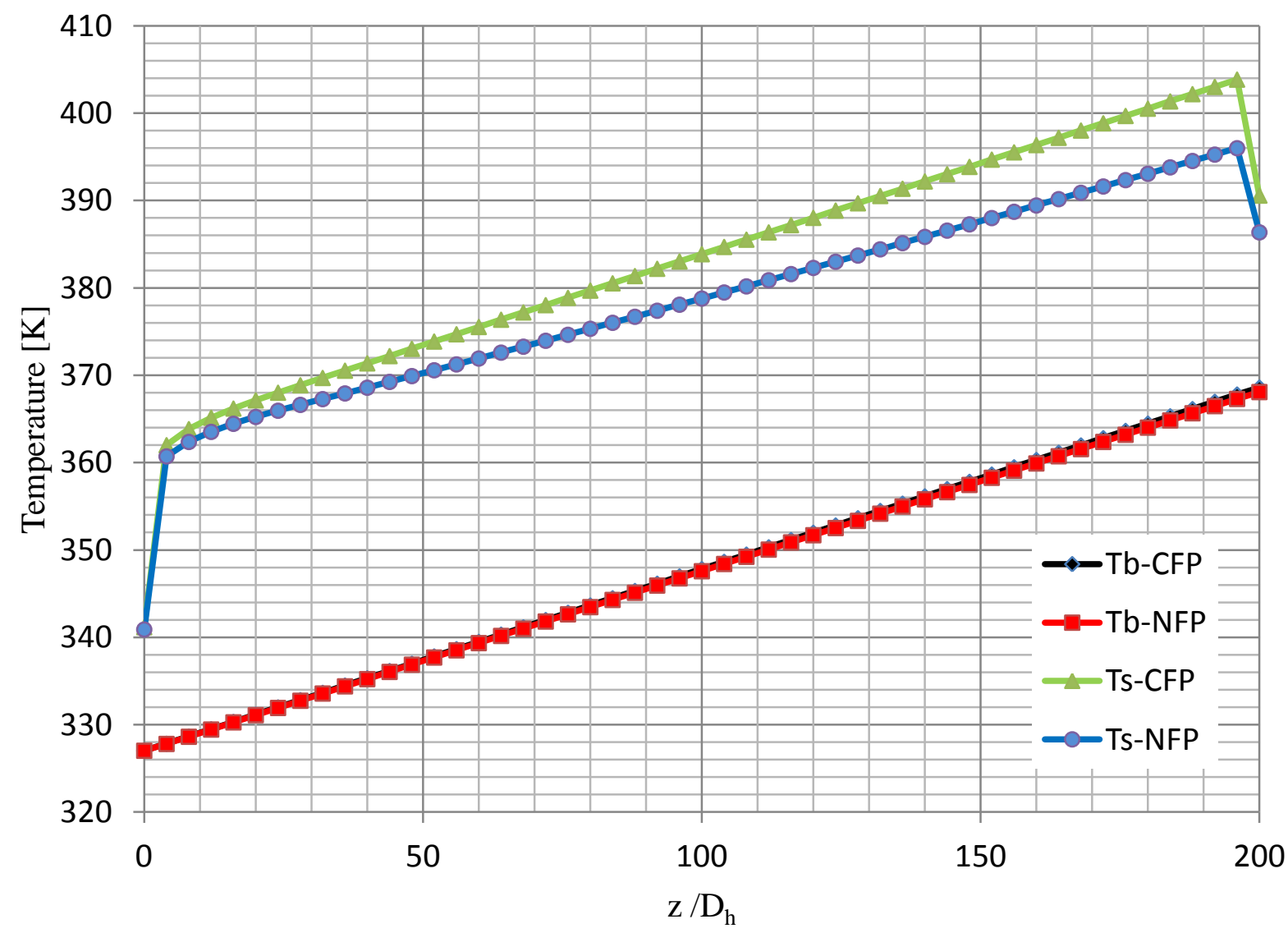

Figure 4-9. Clad Surface Temperatures and Bulk Fluid Temperatures for both the CFP case and NFP case. 
hydraulic diameter of the flow channel, i.e. $2.54 \times 10^{-3} \mathrm{~m}$, in a manner consistent with Equation 4.11 yields a value of 356.58 for the model Nusselt modulus. This value will now be compared with other well known Nusselt correlations for which the system characteristics are consistent.

The Sieder-Tate correlation for the Nusselt modulus has the following form for use under the indicated conditions [9],

$N u_{D}=0.027 \operatorname{Re}_{D}^{4 / 5} \operatorname{Pr}^{1 / 3}\left(\frac{\mu}{\mu_{w}}\right)^{0.14} \quad\left\{\begin{array}{c}0.7 \leq \operatorname{Pr} \leq 16,700 \\ \operatorname{Re} e_{D} \geq 10,000 \\ \frac{z}{D_{h}} \geq 10\end{array}\right.$

Here $R e$ is the Reynolds number and $\operatorname{Pr}$ is the Prandtl number. The Prandtl number is calculated using the following relationship

$\operatorname{Pr}=\frac{\mu C_{p}}{k_{f}}$

This composite structure yields a value of 2.391 for the Prandtl number. The Reynolds number can be calculated using the mass flow rate.

$R e=\frac{\dot{m} D_{h}}{\mu A_{c}}$

Since the model in question uses CFP, the ratio of viscosities in Equation 3.56 is unity.

Substituting relationships 4.13 and 4.14 into 4.12, the Nusselt modulus has the value 369.95.

The Sieder-Tate relationship, Equation 4.12, yields a relative difference of $3.75 \%$ with the Nusselt modulus calculated from the model results. This relative difference is calculated using the following structure

$R D=\left(\frac{N u_{D_{C O M S O L}}-N u_{D_{S T}}}{N u_{D_{C O M S O L}}}\right) \times 100$

Another Nusselt modulus correlation that is compatible with the given model was provided by Gnielinski [9]. The correlation has the following form 
$N u_{D}=\frac{(f / 8)\left(R e_{D}-1000\right) \operatorname{Pr}}{1+12.7(f / 8)^{1 / 2}\left(\operatorname{Pr}^{2 / 3}-1\right)}\left\{\begin{array}{c}0.5 \leq \operatorname{Pr} \leq 2000 \\ 3000 \leq \operatorname{Re} e_{D} \leq 5 \times 10^{6} \\ \frac{Z}{D_{h}} \geq 10\end{array}\right.$

Here $f$ is the Darcy friction factor given by [9]

$f=0.184 R e_{D}^{-1 / 5}\left\{R e_{D} \geq 2 \times 10^{4}\right.$.

The Gnielinski correlation for the Nusselt modulus yields a value of 377.62, which results in a relative difference of $5.90 \%$ with the model Nusselt modulus. The Hausen equation, i.e. Equation 2.3, yields a value of the Nusselt modulus of 372.62. This yields a relative difference of $4.50 \%$ with the model Nusselt modulus.

While the Seider-Tate, the Gnielinski, and the Hausen correlations agree well with the model Nusslet modulus, the information suggests that the Seider-Tate correlation is more representative of the HFIR system. This conclusion will also be drawn for the NFP case.

The NFP case is less straight forward than the CFP case. Given the variable nature of the fluid properties, the Reynold's number and the Prandtl number become distributed in the flow direction. Therefore, a local Nusselt modulus, in terms of the aforementioned correlations, will be distributed in terms of the previously mentioned dimensionless structures. The model Nusselt modulus will be distributed due to the distribution of the local convection coefficient and the thermal conductivity of the fluid.

A choice has to be made, regarding how the fluid properties are to be evaluated for use in calculations, i.e. should the fluid property be evaluated at the local bulk fluid temperature or the local wall temperature? More specifically, what effect does this choice have on the local Nusselt modulus regarding the temperature dependent thermal conductivities? Using Equation 4.11, one can establish a ratio test to gain some qualitative idea of this effect on the local Nusselt modulus. 
$\frac{N u_{D}\left(T_{s}\right)}{N u_{D}\left(T_{b}\right)}=\frac{h_{T b} D_{h}}{k_{f}\left(T_{s}\right)} \frac{k_{f}\left(T_{b}\right)}{h_{T s} D_{h}}$

Using Equation 4.8, i.e. the definition of the convection coefficient, one finds, as expected, that $h_{T b}=h_{T s}$, therefore, Equation 4.18 results in

$\frac{N u_{D}\left(T_{s}\right)}{N u_{D}\left(T_{b}\right)}=\frac{k_{f}\left(T_{b}\right)}{k_{f}\left(T_{s}\right)}$

Using Appendix A with the knowledge that $T_{s}>T_{b}$, one observes that the ratio of thermal conductivities is less than unity, for the heated wall case, and thus $N u_{D}\left(T_{s}\right)<N u_{D}\left(T_{b}\right)$. Physically, since the convection mechanism is driven by thermal phenomena at the heated surface, it stands to reason that the thermal conductivity of the fluid should be evaluated at the heated surface temperature, i.e. $k_{f}=k_{f}\left(T_{s}\right)$.

The Nusselt modulus - Prandtl number (NP) ratio is plotted as a function of the Reynold's number in Figure 4-10. The designation after the colon, in the legend of Figure 4-10, represents the source of the Nusselt modulus, i.e. C COMSOL, G Gnielinski, ST SiederTate, $\mathrm{H} \sim$ Hausen. The model Nusselt modulus for the NFP case is larger in magnitude than the other correlations. This implies that the aforementioned Nusselt moduli provide larger surface temperatures, with the Gnielinski correlation providing the largest surface temperatures of the group. One notices, that again, the Sieder-Tate correlation provides the most similar results to the (COMSOL) model Nusselt number. Figure 4-11 shows the relative difference, i.e. Equation 4.15, between the aforementioned Nusselt correlations and the model Nusselt number.

Figure 4-11 shows that for increasing Reynolds numbers the relative difference between the Sieder-Tate correlation and the model value decreases, again showing that the Sieder-Tate correlation is more representative of the model in question. The lower bound of the relative difference for the Sieder-Tate correlation is $1.2 \%$ for a Reynolds number of $1.32 \times 10^{5}$ and an upper bound of $4.3 \%$ for a Reynolds number of $8.27 \times 10^{4}$. The relative difference between the Hausen correlation and the model Nusselt modulus is shown to increase with increasing 


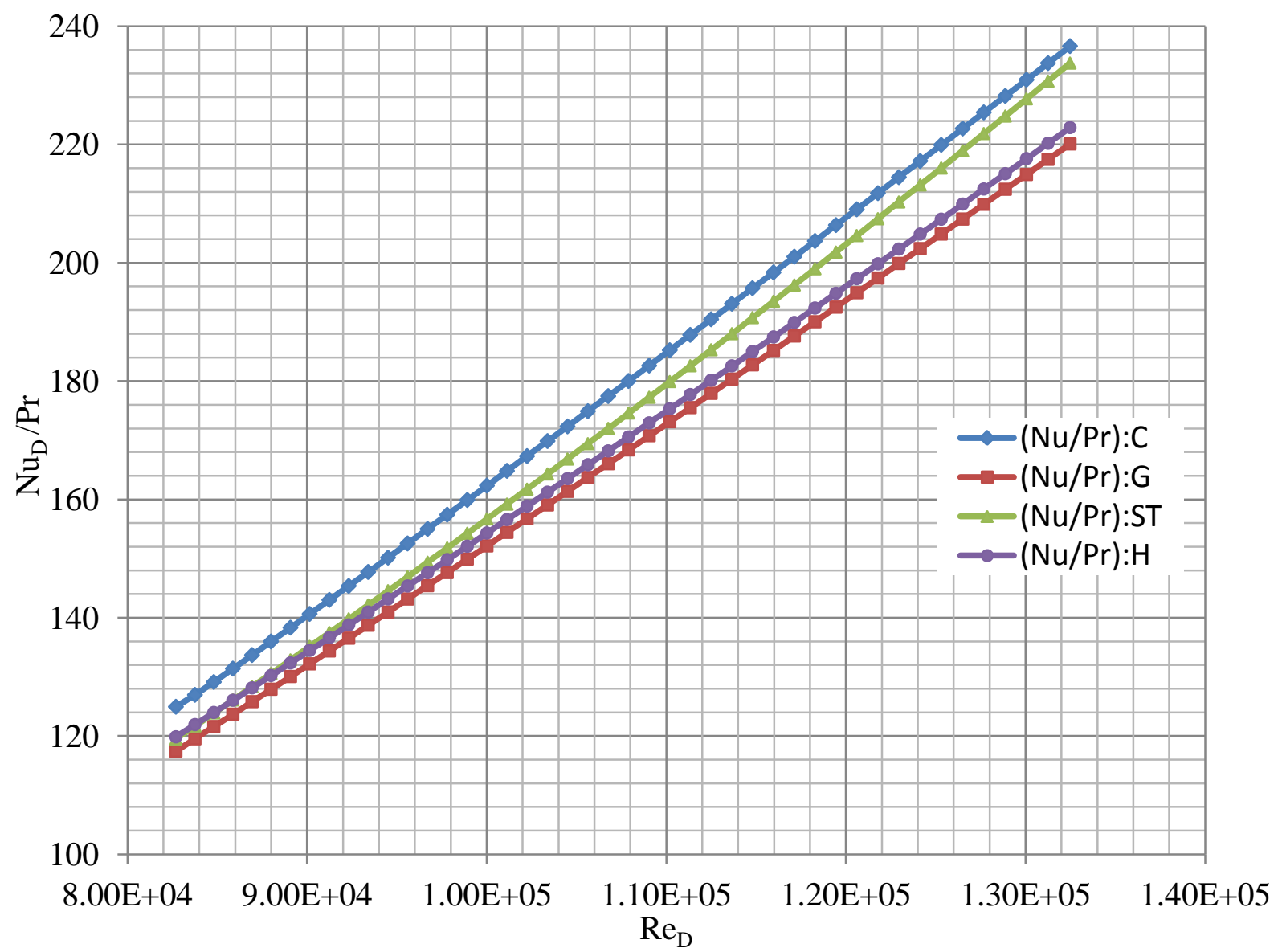

Figure 4-10. Comparison of the Nusselt Modulus - Prandtl Number Ratio as a Function of the Reynold's Number. To clarify the legend: C $\sim$ COMSOL, G $\sim$ Gnielinski, ST $\sim$ Sieder-Tate, H Hausen. 


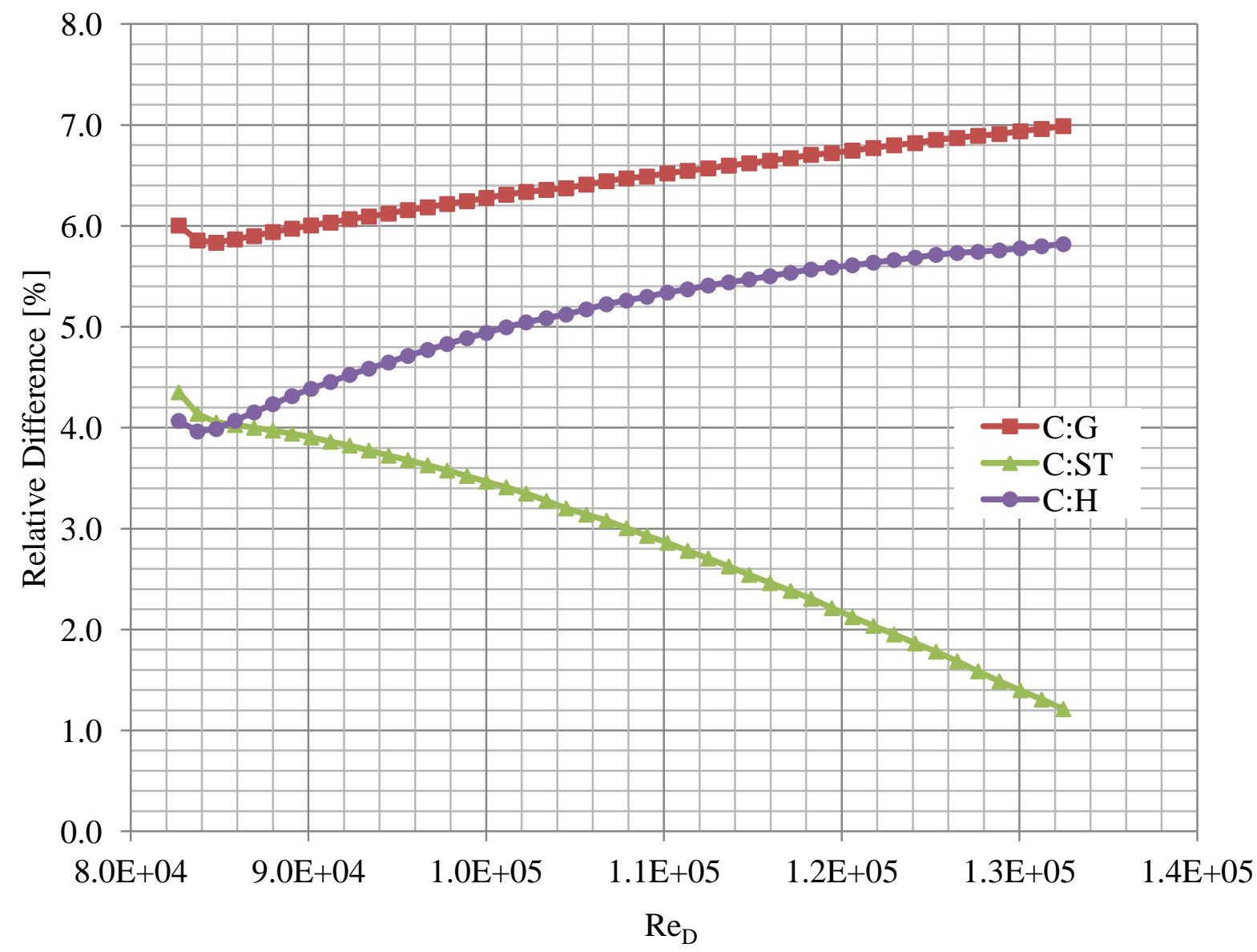

Figure 4-11. Relative Differences of the Gnielinski, Sieder-Tate, and Hausen Correlations compared with the (COMSOL) Model Nusselt Modulus. 
Reynolds number. The relative difference for the Hausen correlation has a lower bound of $3.8 \%$ for a Reynolds number of $8.27 \times 10^{4}$ and an upper bound of $5.9 \%$ for a Reynolds number of $1.32 \times 10^{5}$.

To complete the constant heat flux section, the surface temperature distributions derived from the Gnielinski, Sieder-Tate, and the Hausen correlations compared with the COMSOL determined surface temperature are shown in Figure 4-12 and Figure 4-13 for the CFP case and the NFP case, respectively. For the CFP case, it is observed that the COMSOL determined surface temperature is higher and thus more conservative than those surface temperatures derived from the correlations. This is to be expected since the model Nusselt modulus for the CFP case was lower in magnitude than the correlation values. On the other hand, for the NFP case, the COMSOL determined surface temperature distribution is lower in magnitude than the distributions derived from the correlations. This too was expected since it was shown that the correlations produce Nusselt moduli of lower magnitude than that derived from the model.

\subsection{2-D Turbulent Conjugate Heat Transfer: HFIR Distributed Power Density Case with Constant and Variable Fluid Properties}

The model geometry and boundary conditions for the HFIR distributed power density (DPD) is the same as that used for the constant heat flux case discussed in the previous section, refer to Figure 4-1. The grid structure is identical to that used in the previous section. The DPD imported into the model as an input parameter in the fuel domain is that shown in Figure 4-14. It is important to mention here that the abrupt increase in the DPD shown in Figure 4-14 at $z / D_{h}=100$ is not a physical phenomenon that occurs in the High Flux Isotope Reactor. Instead it is part of a strategy used by the developers of the Steady State Heat Transfer Code to model the thermal consequences of fuel defects. More about this topic will be discussed in Chapter 6 . 


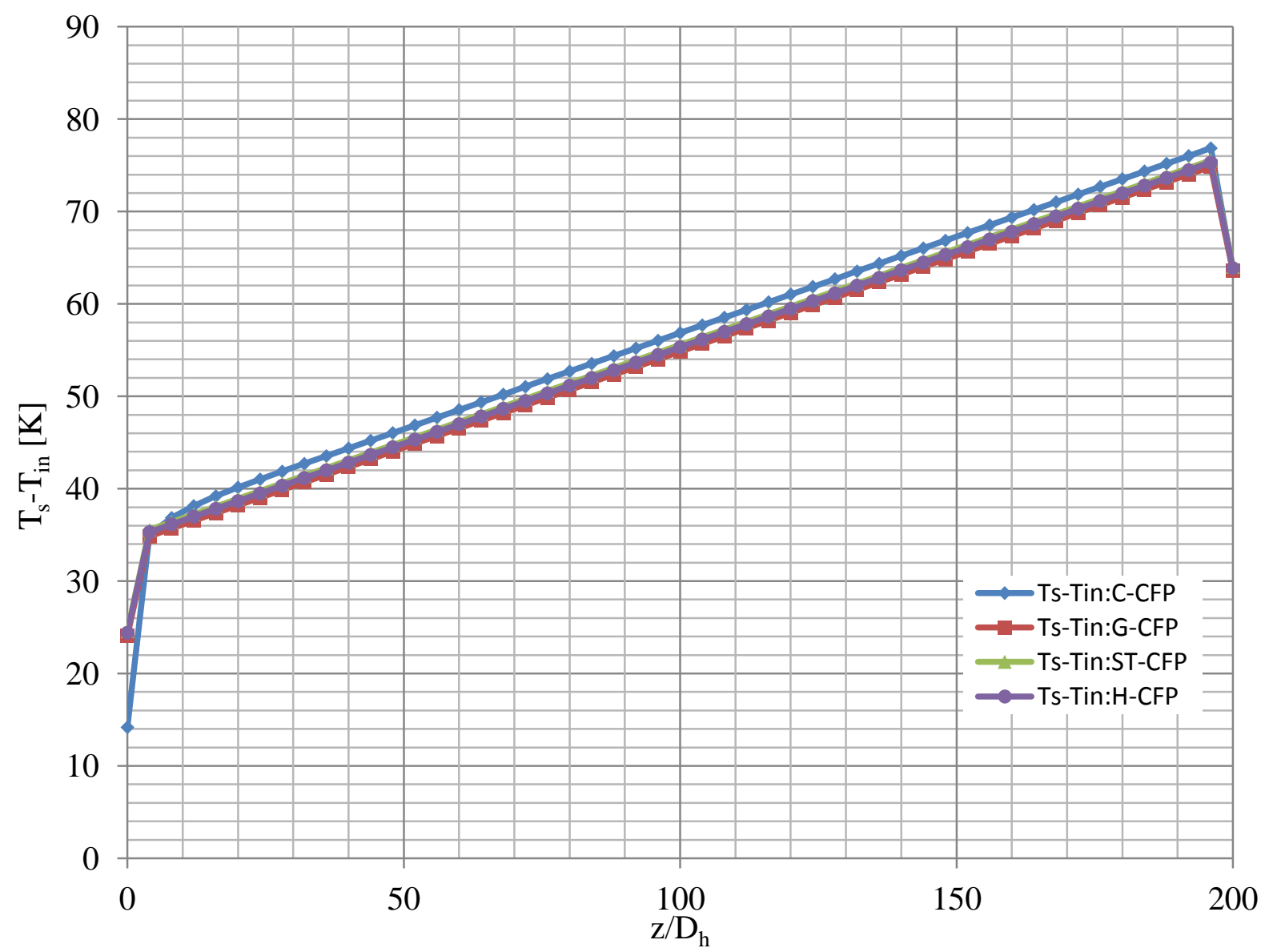

Figure 4-12. Difference Between the Surface Temperature and the Inlet Bulk Fluid Temperature as Derived from the Correlations used for the CFP case. 


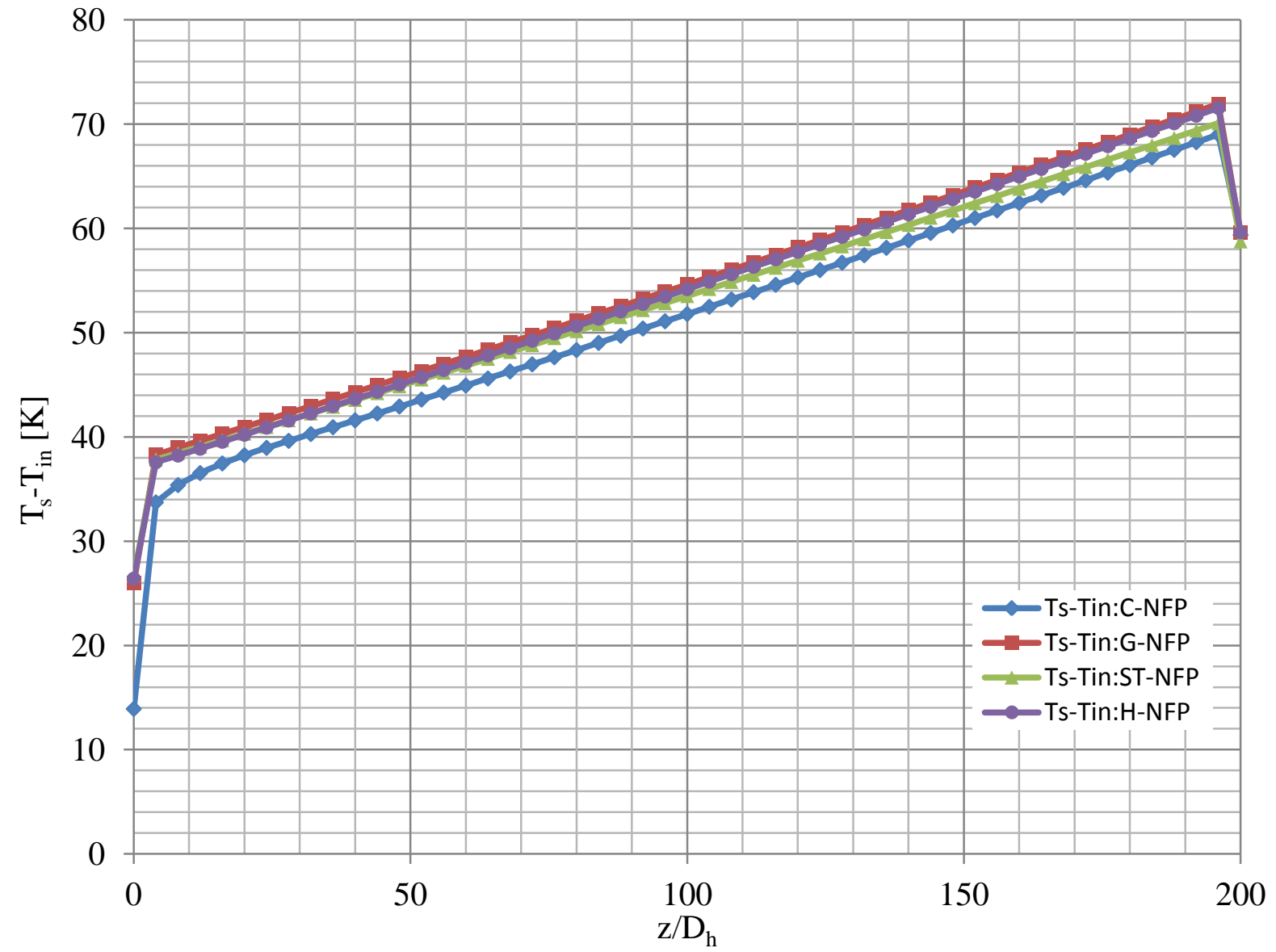

Figure 4-13. Difference Between the Surface Temperature and the Bulk Fluid Inlet Temperature as Derived from the Nusselt Correlations used for the NFP case. 


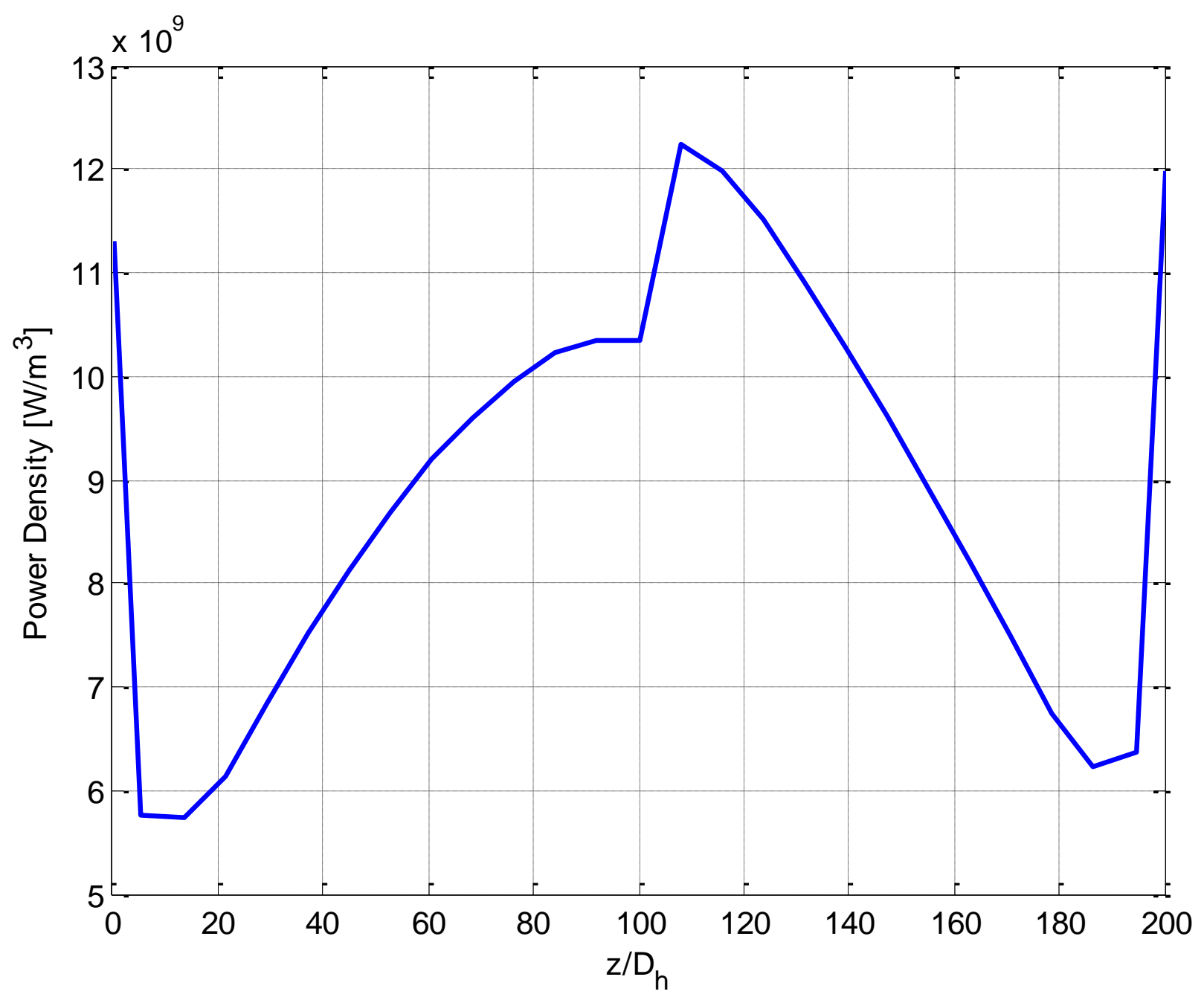

Figure 4-14. Distributed Power Density (DPD) Input for Model. 
The surface heat flux resulting from the use of the DPD is shown in Figure 4-15. Again, the LRN turbulence model is employed due to its ability to resolve thermo-fluid variables within the laminar sublayer of the turbulent boundary layer. The constant fluid properties used for this model are the same as those used in the constant heat flux case, i.e. those shown in Table 4-1. However, the NIST fluid properties will not be the same as those in the constant heat flux case especially those evaluated at the temperature of the heated surface due to its non-linear distribution.

The difference between the clad surface temperatures and the bulk fluid temperatures, or for brevity, the temperature difference, for both the CFP case and the NFP case are shown in Figure 4-16 as a function of the dimensionless flow direction. Again, the temperature difference of the constant fluid property (CFP) case produces a larger magnitude distribution than the temperature difference of the NIST fluid property (NFP) case. This was explained in the previous section, however, the discussion will be recapitulated here. The thermal conductivity of the fluid increases as the fluid temperature increases, refer to Appendix A, therefore, with a higher thermal conductivity more thermal energy is passed to the fluid thus decreasing the surface temperature.

The convection coefficient for both the CFP and the NFP cases are shown in Figure 4-17. Interestingly, the convection coefficient profiles are not too dissimilar from those convection coefficient distributions of the constant heat flux case, compare Figure 4-17 with Figure 4-5. To discuss the shape of the convection coefficient profiles, consider again Equation 4.9.

$\frac{d h}{d z}=\frac{1}{\left[T_{s}(z)-T_{b}(z)\right]} \frac{d q_{s}^{\prime \prime}}{d z}-\frac{q_{s}^{\prime \prime}}{\left[T_{s}(z)-T_{b}(z)\right]^{2}} \frac{d}{d z}\left[T_{s}(z)-T_{b}(z)\right]$.

Since the heat flux is distributed in this model, the first term cannot be neglected. This expression can be written in a more concise manner

$$
\frac{d h}{d z}=\frac{1}{\left(T_{s}-T_{b}\right)^{2}}\left[\left(T_{s}-T_{b}\right) \frac{d q^{\prime \prime}}{d z}-q^{\prime \prime} \frac{d}{d z}\left(T_{s}-T_{b}\right)\right]
$$




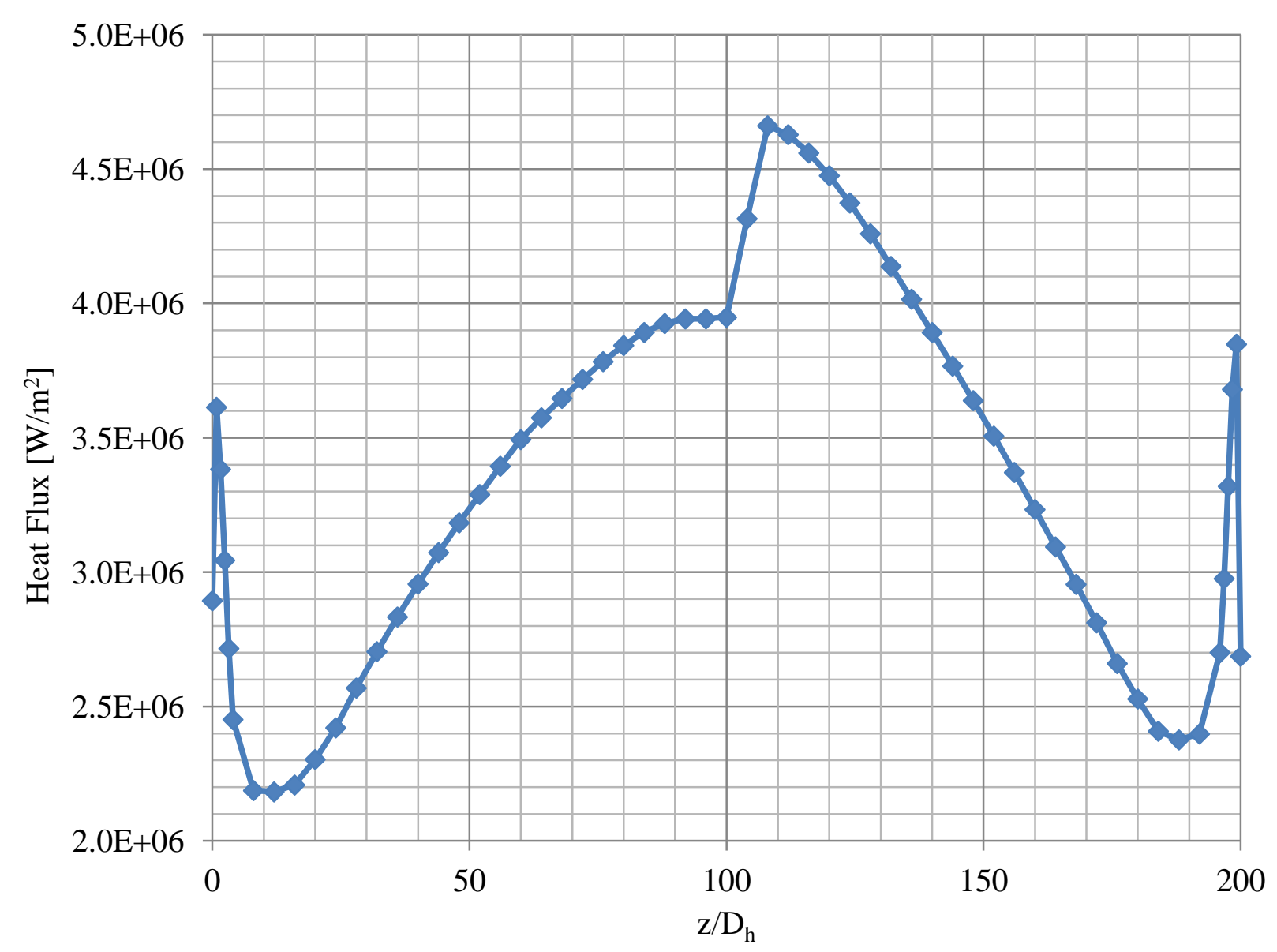

Figure 4-15. HFIR DPD Resultant Surface Heat Flux Distribution. 


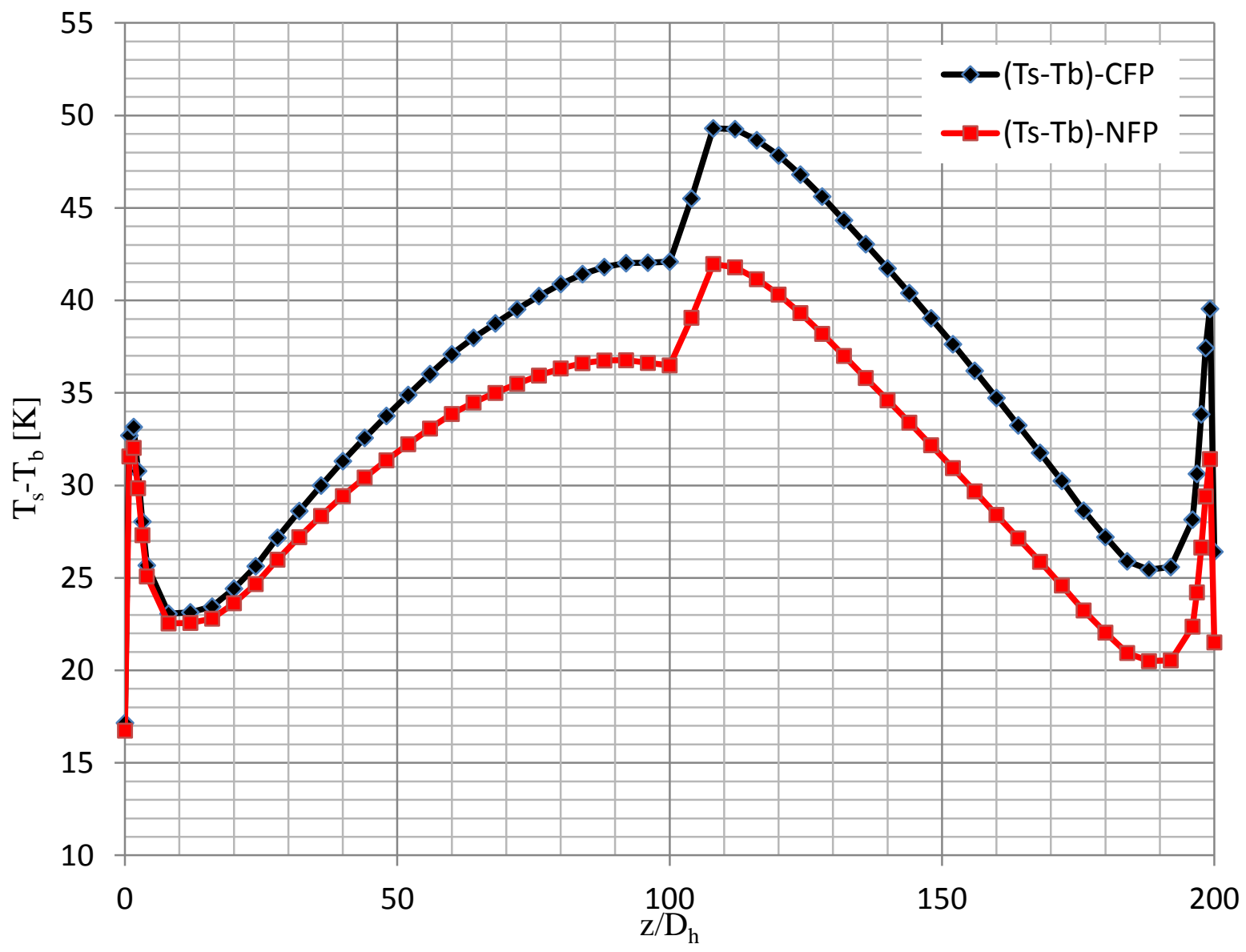

Figure 4-16. Resultant Profiles of the Difference between the Surface Temperature and the Bulk Fluid Temperature. 


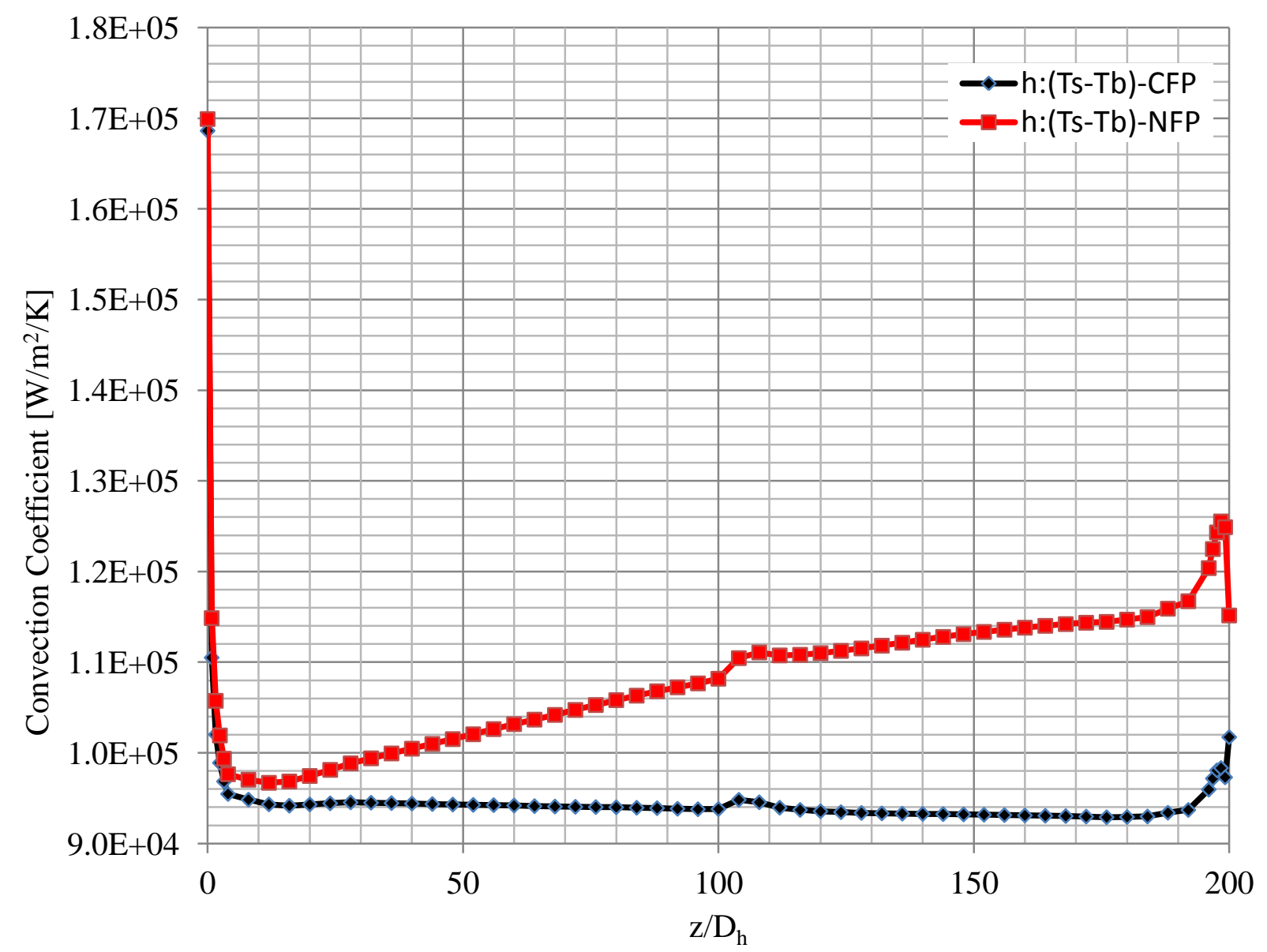

Figure 4-17. Resultant Convection Coefficient for the CFP case and the NFP case. 
Equation 4.20 is slightly easier to read than Equation 4.9. The evaluation of the spatial variation of the convection coefficient shows that deviations in the heat flux profile will have a direct effect on the results. There is a very subtle interplay between the heat flux and the temperature difference established in Equation 4.20. The heat flux has a very similar profile to the temperature difference, i.e. the profiles in question increase and decrease over the same intervals with the exception of the NFP case in the range $90<\mathrm{z} / \mathrm{D}_{\mathrm{h}}<100$ where the temperature difference suffers a slight decrease in magnitude. The magnitudes of the changes, however, are significantly different. By inspection, the maximum changes occur in the entrance and exit regions. Calculation reveals that the maximum change occurs at the entrance

$$
\frac{d q^{\prime \prime}}{d z} \sim O\left(10^{5}\right)\left[\frac{d}{d z}\left(T_{s}-T_{b}\right)\right] ; \quad\left\{\frac{z}{D_{h}} \mid 1.6 \leq \frac{z}{D_{h}} \leq 3.2\right\}
$$

The dominant term in Equation 4.20 is not intuitively obvious, and in fact the dominance switches between terms. This oscillatory dominance will be explained qualitatively. The argument can be made that over the range $10<\mathrm{z} / \mathrm{D}_{\mathrm{h}}<100$, the second term is dominant for the CFP case since all quantities in Equation 4.20 are positive and the convection coefficient decreases in the flow direction as shown in Figure 4-17. For the range $108<\mathrm{z} / \mathrm{D}_{\mathrm{h}}<190$, both derivatives are negative while all other quantities are positive, therefore, the first term is dominant since the convection coefficient continues to decrease over this range. The bump in the convection coefficient, i.e. $100<\mathrm{z} / \mathrm{D}_{\mathrm{h}}<108$, is caused by the first term being dominant with the heat flux derivative being the major contributor. The relative magnitudes of the derivatives in this range are the same as those shown in Equation 4.21.

Figure 4-17 shows that the convection coefficient for the NFP case is an increasing function of the flow direction. In the range $10<\mathrm{z} / \mathrm{D}_{\mathrm{h}}<90$, both derivatives are positive as well as all of the other quantities, therefore, the first term of Equation 4.20 is dominant over this range. For the range $90<\mathrm{z} / \mathrm{D}_{\mathrm{h}}<100$, the heat flux derivative approaches zero while the derivative of the temperature difference decreases slightly which implies that the second term in Equation 4.20 is dominant over this range. The bump occurring in the range $100<\mathrm{z} / \mathrm{D}_{\mathrm{h}}<108$ is 
indicative of a first term dominance due to the large acute increase in both derivatives. Finally, in the range $108<\mathrm{z} / \mathrm{D}_{\mathrm{h}}<190$, both derivatives are negative which implies that the second term in Equation 4.20 dominates the derivative of the convection coefficient.

The value of the model average Nusselt number for the HFIR DPD CFP model is calculated to be 358.05 using Equation 4.11. The model Nusselt number is different than that recorded in the constant heat flux CFP case due to the decreasing nature of the convection coefficient shown in Figure 4-17. Since the property values are identical to those used in the constant heat flux CFP case, the values of the different correlations, i.e. Seider-Tate, Gnielinski, and Hausen, are also identical to those calculated from those results. To refresh one's memory the values follow: $N u_{D}=369.95$ (Sieder-Tate: Equation 4.12), $N u_{D}=377.62$ (Gnielinski: Equation 4.16), and $N u_{D}=372.62$ (Hausen: Equation 2.3). Since the CFP case results in single value Nusselt numbers from the above mentioned correlations, the average Nusselt number for the model will be compared instead of the instantaneous value. The Seider-Tate correlation results in a difference of $3.32 \%$ with the model value. This difference is calculated using Equation 4.15. The Gnielinski correlation results in a difference of $5.46 \%$, and the Hausen correlation results in a difference of $4.07 \%$.

The values of the Nusselt number found from the above correlations divided by the Prandtl number are compared with the model Nusselt number-Prandtl number ratio for the NFP case in Figure 4-18. First, one notices the bump in the model curve, i.e. (Nu/Pr):C, that occurs over the range $1.02 \times 10^{5} \leq z / D_{h} \leq 1.08 \times 10^{5}$. This is a relic of the way in which this information was constructed. It was explained earlier, with the aid of Equation 4.20, that the convection coefficient suffers an acute increase in magnitude, over this same range, due to the relative magnitudes of the derivatives in the flow direction of both the heat flux and the temperature difference. It was shown that the derivative of the heat flux over this range was five orders of magnitude larger than the derivative of the temperature difference. Therefore, one expects to see this effect reflected in the convection coefficient and, by extension, in the Nusselt number since neither the hydraulic diameter nor the thermal conductivity have the capacity to mitigate this effect. Further, one notices that the Hausen correlation is more representative of the 


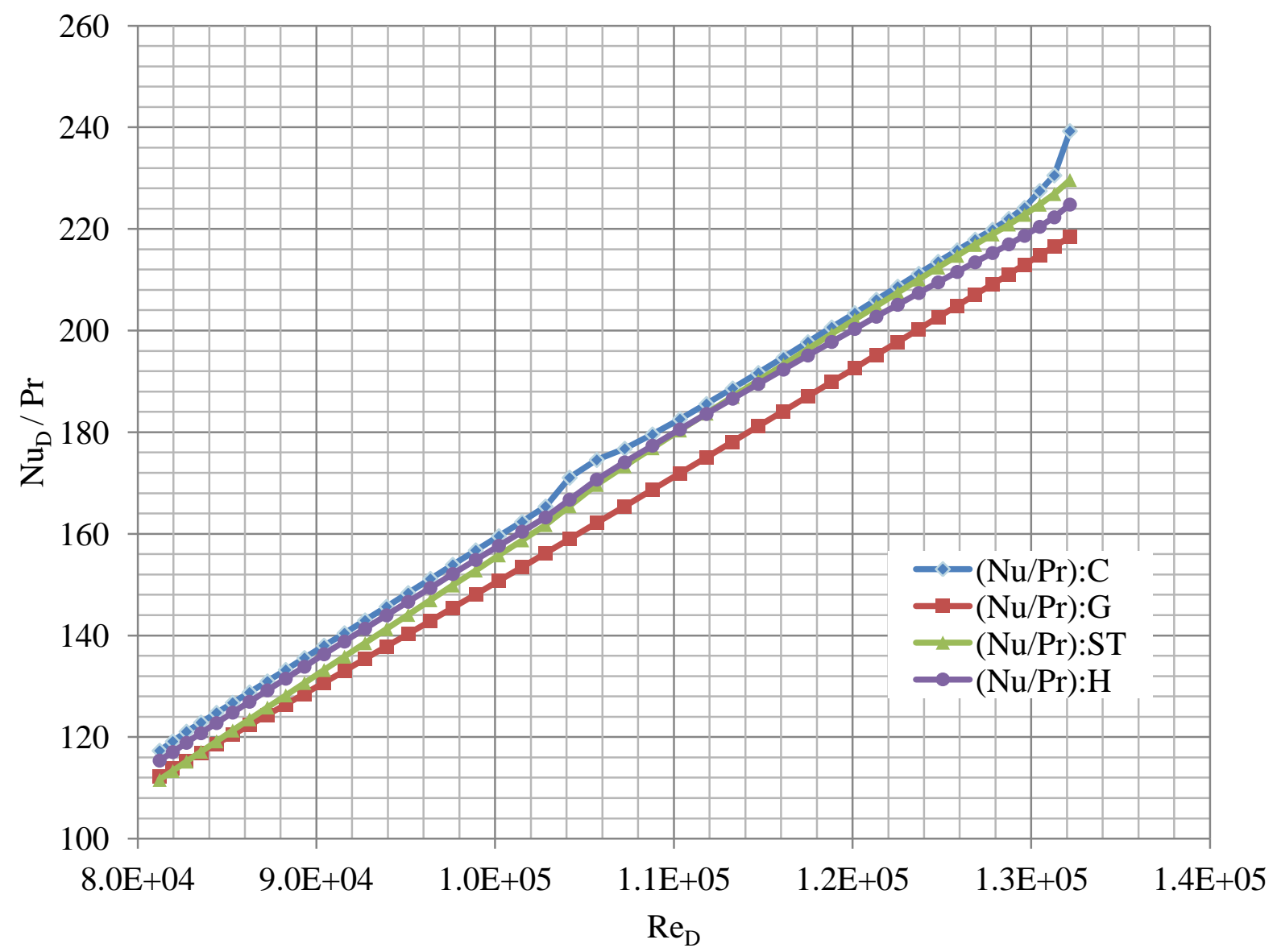

Figure 4-18. Nusselt Number Correlation Results Compared with the Model Nusselt Number for the NFP. 
model Nusselt number in the range $82,000 \leq R e \leq 113,000$. It is interesting to note that of all the cases considered to this point the, Seider-Tate correlation was the better fit. However, the most physically representative model, i.e. the HFIR DPD NFP case, yields a conclusion similar to that of Gambil and Bundy [10], at least within the above mentioned range of Reynolds numbers, that the Hausen correlation provides the most accurate representation of the Nusselt number. Toward the upper end of the Reynolds number range, i.e.113,000 $\leq R e \leq 130,000$, again, it is the Seider-Tate correlation that matches best.

The relative differences between the model Nusselt number distribution and the Nusselt number correlations are shown in Figure 4-19. Again, the relative difference was constructed using Equation 4.15. The large upturns that occur in each curve, i.e. $R e \geq 130,000$, are due to the increase of the Nusselt-Prandtl ratio found in Figure 4-18. This effect is based on the same argument as that presented for the acute increase in the convection coefficient, i.e. the magnitude of the derivative in the heat flux is much larger than the magnitude of the derivative in the temperature difference. This effect permeates all subsequent calculations.

From Figure 4-19, the Gnielinski correlation produces the largest consistent difference over the range of Reynolds numbers presented. Therefore, the Gnielinski correlation will produce the larger surface temperatures than the other correlations. It is recommended here that, due to its production of higher surface temperatures in these model configurations, the Gnielinski correlation be used for less detailed models, i.e. models where the influence of the flow field is not directly modeled, which employ variable fluid properties with the LEU fuel as the thermal source. The Gnielinski correlation has been consistent in producing lower values of the Nusselt number for the NFP cases considered in this document.

Figure 4-19 shows that the Hausen correlation produces values within $2 \%$ of the model NFP case over most of the range of Reynolds number considered with the exception of the acute increase in the convection coefficient which, in Figure 4-18, occurs in the range 103,000 $\leq$ $R e_{D} \leq 108,500$. Further, the Hausen correlation is more consistent, i.e. maintaining its value, with the model Nusselt-Prandtl ratio than the Sieder-Tate correlation. The Seider-Tate 


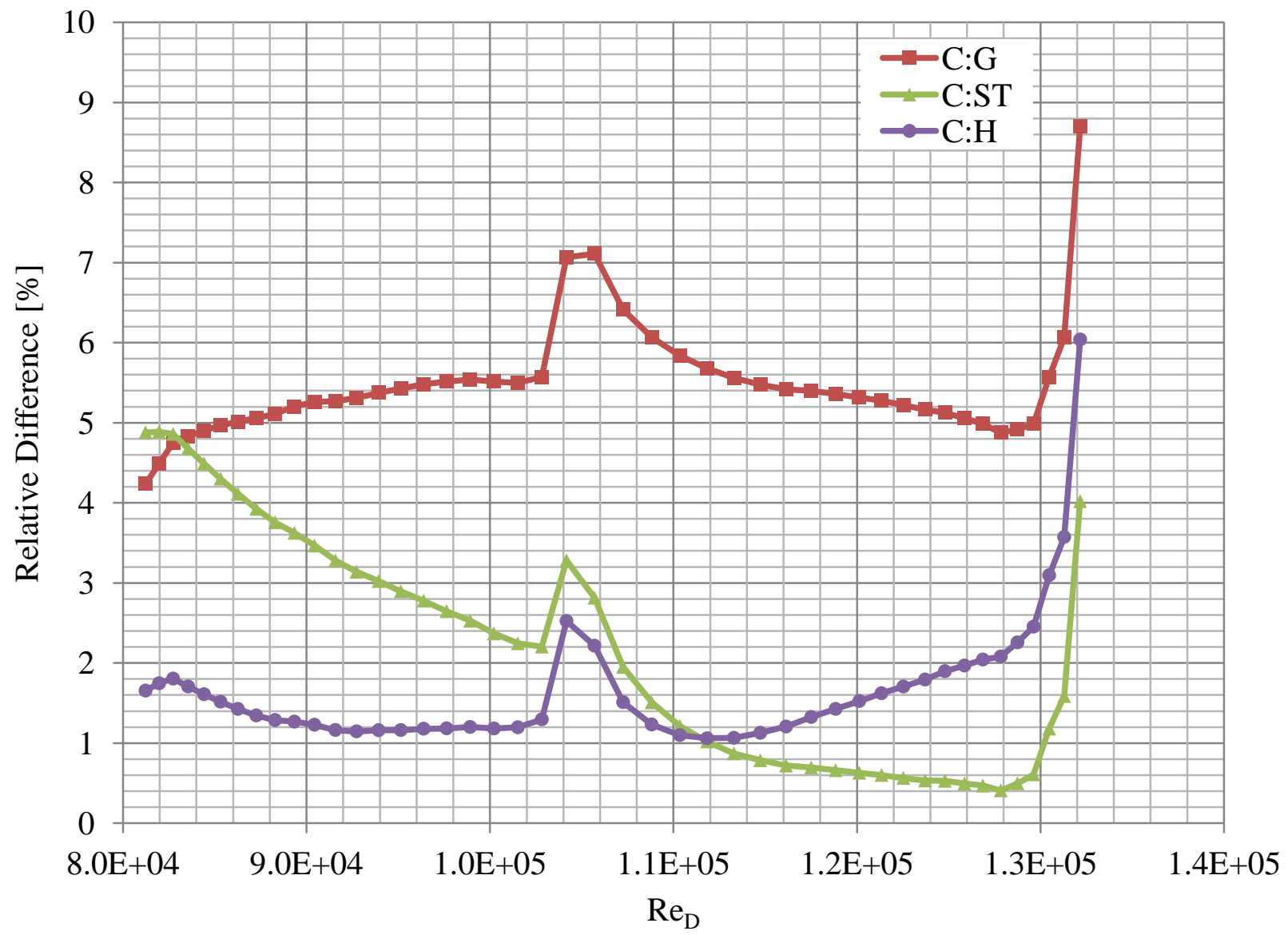

Figure 4-19. Relative Differences of the Gnielinski, Sieder-Tate, and Hausen Correlations compared with the Model Nusselt Modulus. 
correlation begins, i.e. on the low end of the Reynolds number scale, at approximately $4.8 \%$ compared with $2.6 \%$ for the Hausen correlation. The relative difference of the Sieder-Tate correlation with the model decreases with the Reynolds number overall. At a value of $R e \cong$ 112,000 the relative differences of the Sieder-Tate correlation and the Hausen correlation are equal, and subsequent values of the Seider-Tate correlation produce values more representative of the model.

The temperature profiles for the CFP case generated from the Seider-Tate, Gnielinski, and Hausen correlations are shown in Figure 4-20 compared with the model CFP temperature distribution. As with the constant heat flux CFP case, the model temperature distribution is larger in magnitude than the distributions derived from the correlations. The Sieder-Tate and Hausen correlation generated temperature distributions are almost coincident and lie above the Gnielinski correlation generated distribution.

The temperature distributions for the NFP case generated from the same Nusselt number correlations are shown in Figure 4-21 compared with the model NFP temperature distribution. As in the constant heat flux NFP case the model temperature distribution is lower in magnitude than the correlation generated temperature distributions. As stated earlier, the Gnielinski correlation generated temperature distribution is the most conservative of the correlations considered in this document. The Hausen correlation generated temperature distribution matches the model distribution very well over the entire range. It is observed that the Sieder-Tate correlation generated temperature distribution also matches the model distribution, however, there exists more of a deviation in the range $0 \leq z / D_{h} \leq 100$ than that produced by the Hausen distribution.

It has been shown that the model temperature distribution for the HFIR distributed power density with NIST fluid properties is closely matched by the Hausen distribution, i.e. the Hausen generated temperature distribution. One should recall that the Hausen correlation was chosen based on experimental data regarding turbulent flow in thin channels performed by Gambil and Bundy [10]. Here it is shown that the Hausen correlation is very well matched with the 


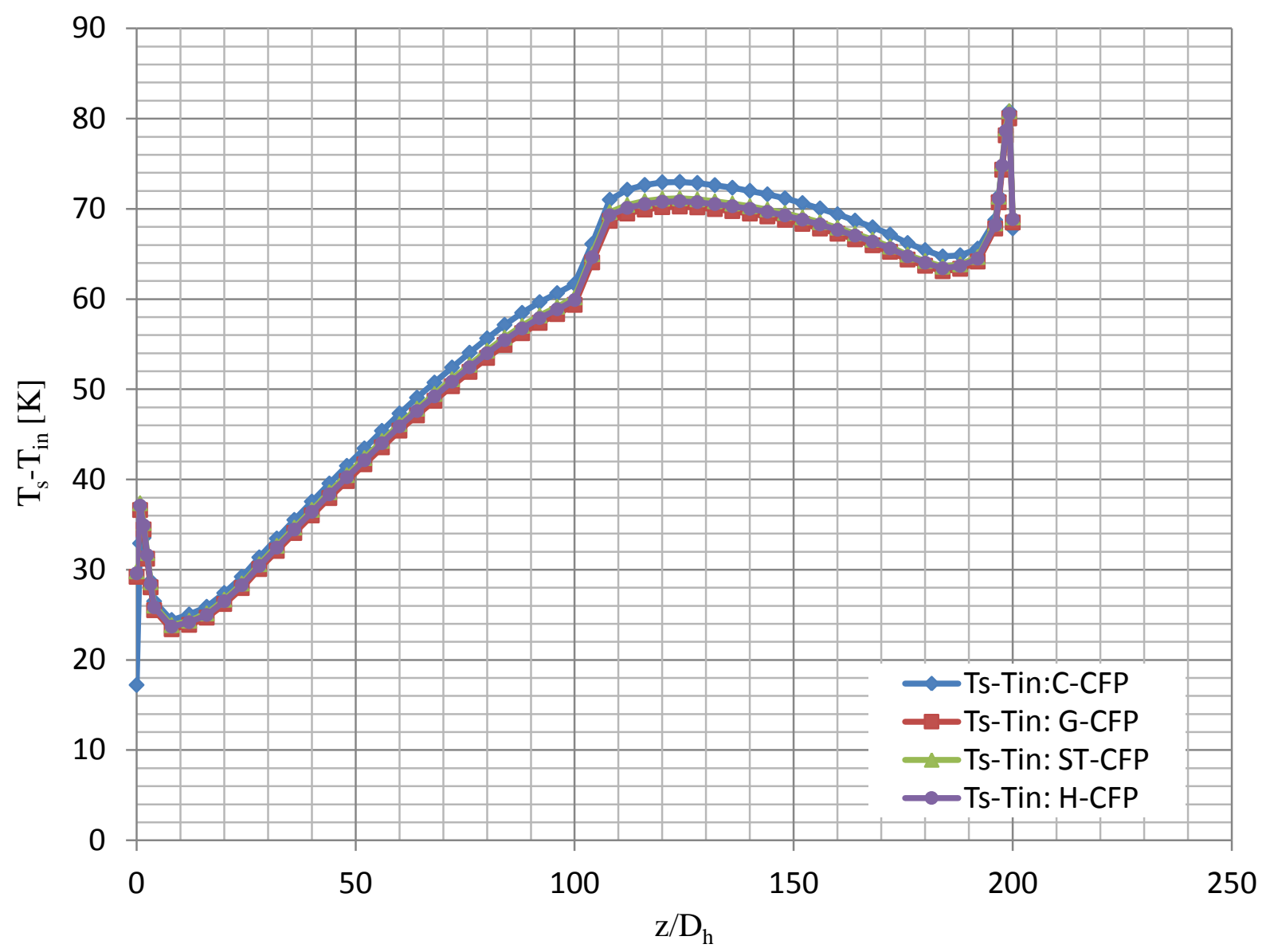

Figure 4-20. Temperature Distribution for the CFP case Generated by the Gnielinski (G-CFP), Sieder-Tate (ST-CFP), and the Hausen (H-CFP) Correlations Compared with the Model Temperature Distribution (C-CFP). 


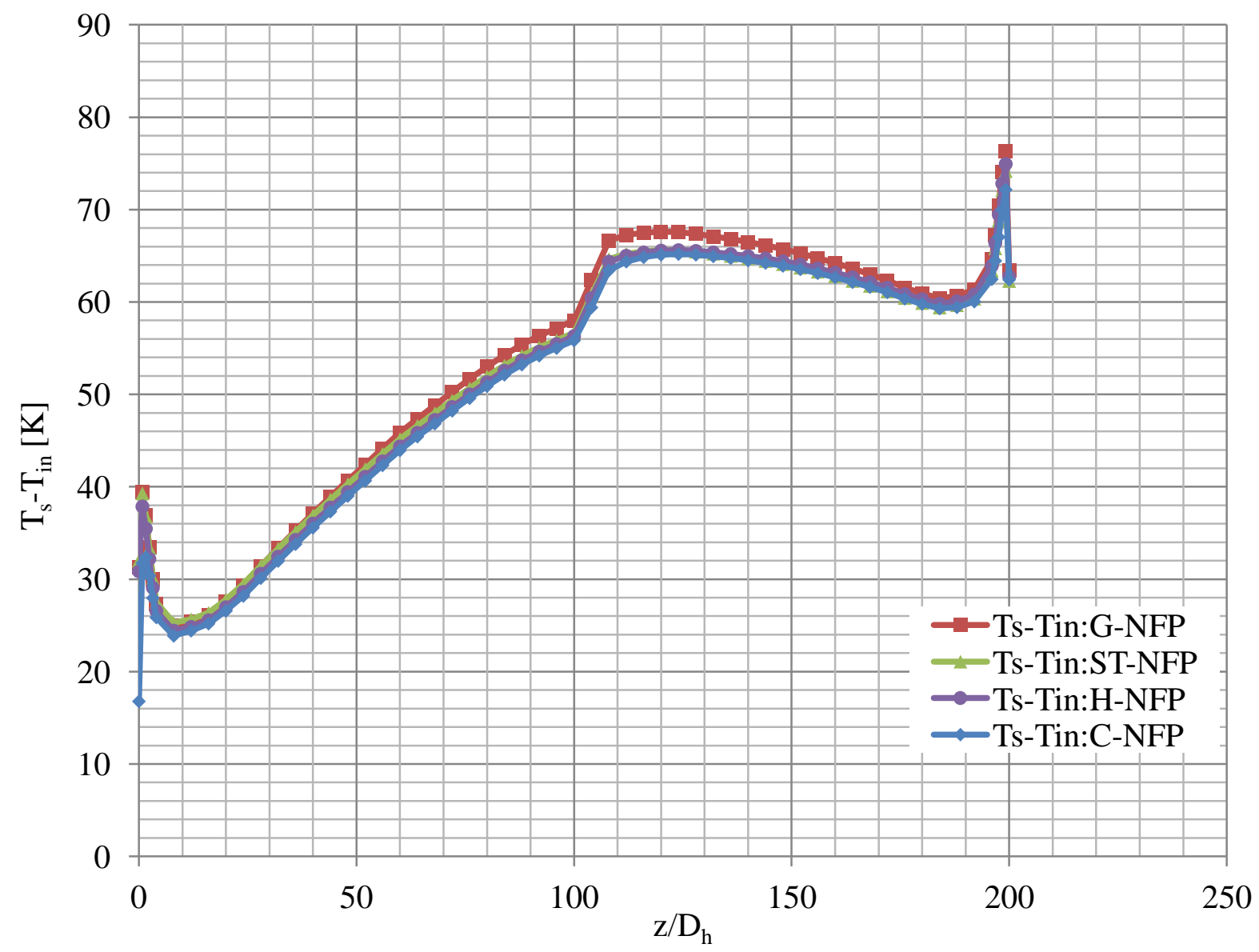

Figure 4-21. Correlation Generated Temperature Distributions Compared with the Model Distribution for the NFP Case. 
computational model. Therefore, the model produces surface temperature distributions in accordance with the experimentally determined correlation.

\subsection{Conclusions for the 2-D Modeling Case}

The 2-D modeling case provides a useful test bed within which to investigate the heat transfer characteristics associated with different scenarios, e.g. distributed heat flux with constant fluid properties compared with variable fluid properties. This 2-D investigation suggests that the constant fluid property (CFP) case provides higher clad surface temperatures thus decreasing the thermal margin, i.e. $T_{\text {sat }}-T_{s}$, defined here as the difference between the saturation temperature of the fluid and the surface temperature of the clad. It was shown that for the CFP case, for both constant and distributed heat flux profiles, that the convection coefficient remained relatively constant. This information provides confidence in the results of a thermal diffusion code which employs constant convective boundary conditions provided that the appropriate magnitude of the convection coefficient is used. The convection coefficient can be appropriately determined from the Sieder-Tate relationship shown in Equation 4.12 given that the thermal-fluid conditions are consistent with the use of this relationship. 


\section{Chapter 5 3-D Model Development: Advanced Neutron Source Reactor Thermal Hydraulic Test Loop Model}

In this chapter the development of the 3-D Advanced Neutron Source Reactor Thermal Hydraulic Test Loop (ANSR THTL) model, used to aid in the validation of COMSOL with experimental data, is discussed. The test conditions from the ANSR THTL have been used as inputs into the 3-D model. The results from the COMSOL model, developed in version 4.3a, were post processed and compared with the experimental results.

"The Thermal Hydraulic Test Loop (THTL) was a facility for experiments constructed to support the development of the Advanced Neutron Source Reactor (ANSR)" [23] [24]. "The facility was built to simulate a full length coolant subchannel of the ANSR core, allowing experimental determination of thermal limits under expected ANSR thermal hydraulic conditions" [23] [24].

Experimental data from the ANSR thermal-hydraulic test loop (THTL) will be used in the code validation effort. The ANSR THTL presents a unique experimental data set that has similar characteristics to that of the flow channels in the HFIR core, i.e. high surface heat flux and a long narrow coolant channel. The aspect ratio of the THTL coolant channel, length to thickness, is 399 , which is only $16.9 \%$ less than that of the HFIR. Flow velocities through the THTL range from $62.34 \mathrm{fps}(19.00 \mathrm{~m} / \mathrm{s})$ to $88.58 \mathrm{fps}(27.00 \mathrm{~m} / \mathrm{s})$ which are also comparable to, albeit larger than, the flow velocity characteristic of the HFIR which, as previously stated, is approximately $52.00 \mathrm{fps}(15.85 \mathrm{~m} / \mathrm{s})$ at steady state operation. The average and peak heat fluxes produced in the THTL are stated to be $6 \mathrm{MW} / \mathrm{m}^{2}$ and $12 \mathrm{MW} / \mathrm{m}^{2}$, respectively [23]. The average heat flux through the HFIR core is calculated to be $2.13 \mathrm{MW} / \mathrm{m}^{2}$. Therefore, with higher flow velocities and higher average heat fluxes, the THTL provides extreme cases, relative to HFIR conditions, with which to validate the code. 


\subsection{ANSR THTL Model Geometry and Boundary Conditions}

The cross section of the geometry of the THTL is shown in Figure 5-1. The flow channel gap has the same thickness as the HFIR flow channel gap, i.e. $1.27 \mathrm{~mm}$. Also, the solid material creating the heat transfer surface and thermal energy generating medium is made of the same material as the HFIR fuel plate cladding, i.e. Al 6061. The reduced wall thickness at the curved ends of the flow channel was designed to reduce heat flux peaking on the ends of the flow channel [23]. This cross section is extended through $507 \mathrm{~mm}$ in the flow direction. To expedite the computation, the model geometry was reduced to one-quarter of the entire geometry. The quarter section used is indicated by the red dashed line in Figure 5-1. The red dashed lines represent heat transfer and flow symmetry planes. The boundary conditions established at these symmetry planes are thermal and flow symmetry conditions.

The full model of the ANSR THTL is shown in Figure 5-2, i.e. the extruded cross section. It is important to bear in mind that only a quarter of this model was used in the COMSOL environment. The entrance extension is $30.7 \mathrm{~mm}$ long in the flow direction. The first $18.0 \mathrm{~mm}$ was not part of the THTL, but is an artificial extension constructed to ensure that the flow entering the active region of the model is hydrodynamically fully developed. The remaining $12.7 \mathrm{~mm}$ outside of the active region provides a location for upstream pressure measurement consistent with the experimental setup [23] [24]. The exit extension is $12.7 \mathrm{~mm}$ long and was constructed to provide a location for downstream pressure measurement consistent with the experimental setup. Therefore, the global exit of the model represents the location of the downstream pressure tap in the experimental loop. The experimental data used for validation is labeled FE306B03 in the set supplied from ORNL.

Joule heating was used in the experimental setup by supplying a potential difference across the test section in the axial direction. The power input was recorded and the amount of heat loss through the exterior lateral surfaces was measured. The difference between these quantities is the amount of heat passing into the test section and was given the label TS Power. The computational model uses the value of the TS Power as input for the volumetric heat generation rate within the $\mathrm{Al} 6061$ domain. This power density is uniform throughout the solid domain. As a result, the heat flux too will be uniform in the computational domain. Therefore, 


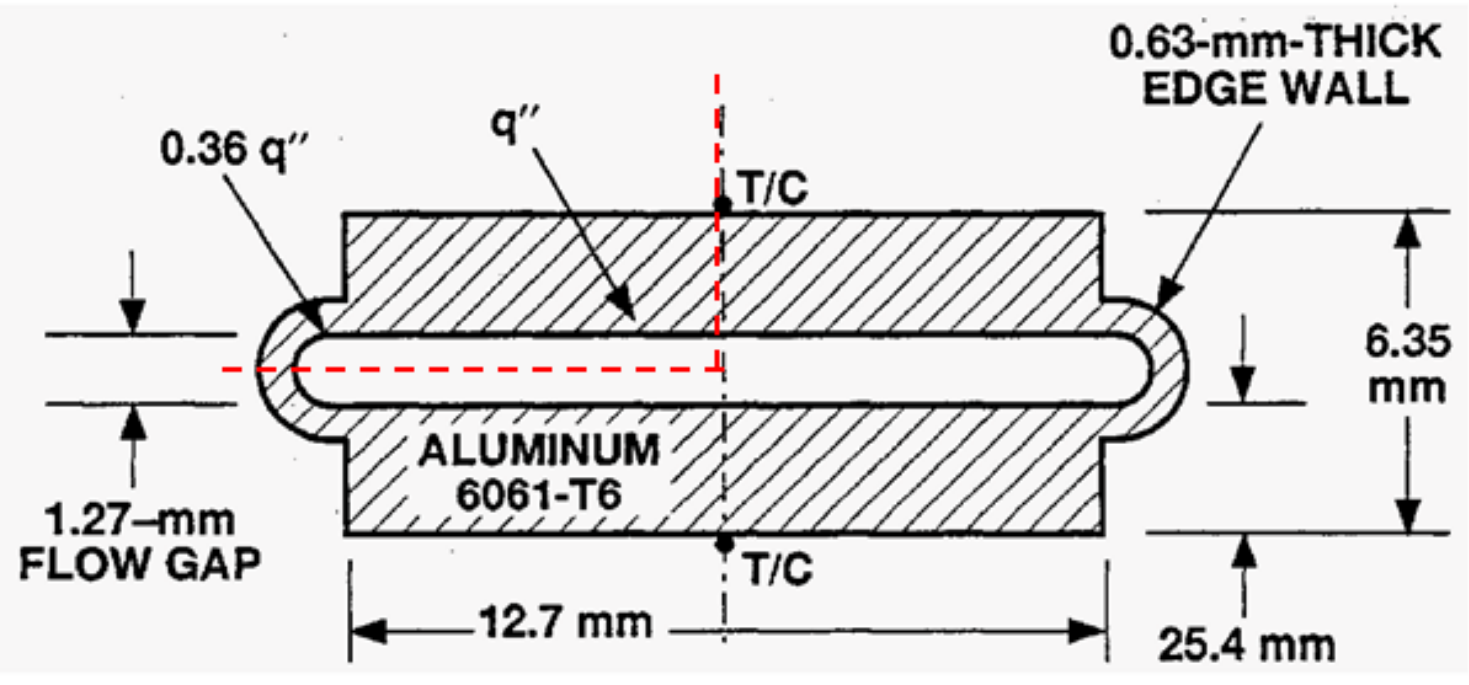

Figure 5-1. ANSR THTL Test Loop Cross Section. The label T/C represents thermocouple locations along the test section. The red dashed lines represent the symmetry boundaries used in the creation of a quarter geometry to simplify the computational effort. 


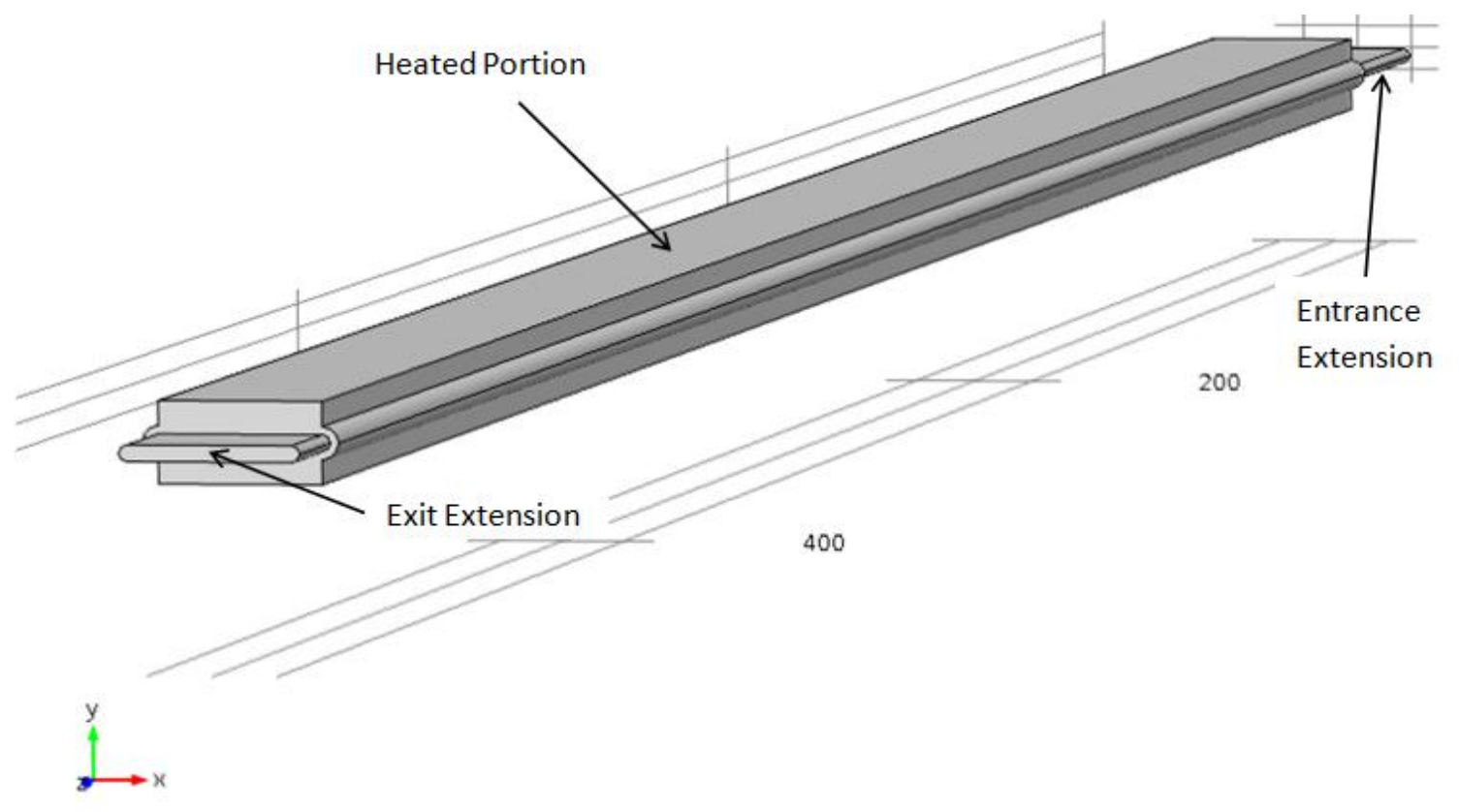

Figure 5-2. Isometric View of the ANSR THTL Model. 
one expects the results of the THTL model to be similar to the constant heat flux NFP case discussed in the last chapter.

The inlet to the computational domain is isothermal with a temperature of $45.45^{\circ} \mathrm{C}$ which is consistent with the experimental setup. Since no thermal sources exist between the global entrance and the active section of the model, one expects that the flow will remain isothermal in this region of the model. The inlet velocity is set to a uniform profile with a magnitude of 21 $\mathrm{m} / \mathrm{s}$. The global outlet pressure was set with a uniform magnitude of $1.698 \mathrm{MPa}$ which is consistent with the experimental data and a convective flow condition for thermal variables. The lateral boundaries of the model are adiabatic since the power supplied to the test section is already adjusted to account for lateral losses. The iterative convergence criterion was set to $1 \times 10^{-6}$ and all grid structures to be discussed have converged with respect to this criterion.

\subsection{Computational Grid Structure Development}

Three different grid structures were created for the simulation. The coarsest grid structure has a total of 82,200 elements. The cross sectional grid structure, i.e. normal to the axial direction, for both the flow channel and solid domain, has an element density of

$\rho_{\text {ele }}=\frac{2}{6.35 \times 10^{-4}}$

Each boundary which makes up the cross section in the model geometry has the number of elements prescribed by the following relationship

$n_{c s}=s * \rho_{\text {ele }} * B L$.

Here $n_{c s}$ is the number of elements in the cross section, $B L$ is the individual cross sectional boundary length and $s$ is a constant adjustment factor used to construct finer grid structures in as much a consistent way as possible. The axial grid structure is created by sweeping the cross sectional grid structure in the axial direction. This portion of the grid structure is constructed using the relationship 
$n_{\text {axial }}=s * 200$

The boundary layer grid structure is constructed by specifying the number of layers as

$n_{\text {Layer }}=s * 25$

The total number of elements, $n$, is given by

$n=\left(n_{c s}+n_{\text {Layer }}\right) n_{\text {axial }}$

The stretching factor used in the boundary layer grid construction was set to 1.25 , which as stated in Chapter 3, increases the adjacent quadrilateral element size by $25 \%$ in the direction normal to the boundary. The thickness adjustment factor was different for the finest grid structure, being set to 2.5 instead of 0.05 , due to errors occurring during grid generation.

The adjustment constant, $s$, was given values of $0.75,1.1$, and 1.77 to produce a total number of elements, $n=82,200 ; n=222,222$; and $n=496,154$, respectively. These particular grid structures were chosen to comply with the Grid Convergence Index calculation to be performed on the model results. This will be discussed in detail shortly. It is important to mention that the grid structure described above is the result of many months of trial and error. Many different grid structures were conceived and analyzed, however, energy balance issues for the NFP cases prohibited their use, i.e. between $13 \%$ and $15 \%$ error. The structures described above represent an error in the balance of energy between $6.83 \%$ and $9.96 \%$.

\subsection{Comparison of Model Results with Experimental Data}

The FE306B03 data and the model results are shown in Table 5-1. Table 5-1 shows the quantity being measured in the left hand column. The results for each grid structure are given below its column heading and the relative error associated with these results are presented in the adjacent column. The relative error is calculated by using the relationship

Error $=\left|\frac{\psi_{\text {comp }}-\psi_{\text {exp }}}{\psi_{\text {exp }}}\right| \cdot 100$ 
Table 5-1. FE306B03 Experimental Data Compared with the Model Results.

\begin{tabular}{|l|r|r|r|r|r|r|r|}
\hline Quantity & Experiment & $\underline{\mathbf{n}=\mathbf{8 2 , 2 0 0}}$ & $\underline{\text { Error[\%] }}$ & $\underline{\mathbf{n}=\mathbf{2 2 2}, \mathbf{2 2 2}}$ & Error[\%] & $\underline{\mathbf{n}=\mathbf{4 9 6 , 1 5 4}}$ & Error[\%] \\
\hline Tout $\left({ }^{\circ} \mathrm{C}\right)$ & 163.09 & 165.413 & 1.424 & 164.642 & 0.952 & 165.021 & 1.184 \\
\hline Pin $(\mathrm{MPa})$ & 2.693 & 2.508 & 6.870 & 2.492 & 7.464 & 2.701 & 0.297 \\
\hline $\begin{array}{l}\text { TS Power } \\
(\mathrm{W})\end{array}$ & 173459 & 173835 & 0.217 & 173762 & 0.175 & 173717 & 0.149 \\
\hline $\begin{array}{l}\text { Max q" } \\
\left(\mathrm{MW} / \mathrm{m}^{2}\right)\end{array}$ & 13.514 & 13.01 & 3.729 & 13.061 & 3.352 & 13.506 & 0.059 \\
\hline $\begin{array}{l}\text { Ave q" } \\
\left(\mathrm{MW} / \mathrm{m}^{2}\right)\end{array}$ & 11.875 & 11.553 & 2.712 & 11.748 & 1.069 & 11.688 & 1.575 \\
\hline Ave v (m/s) & 21 & 20.604 & 1.885 & 20.704 & 1.409 & 20.678 & 1.535 \\
\hline$\Delta \mathrm{p}(\mathrm{Mpa})$ & 0.995 & 0.81 & 18.593 & 0.794 & 20.201 & 1.003 & 0.804 \\
\hline
\end{tabular}

The model outlet bulk temperature was evaluated using Equation 3.55. The outlet bulk temperature was higher than the experimentally measured value for all grid structures. The outlet bulk temperature for the 222,222 element grid produced the smallest error with a magnitude of $0.95 \%$ as shown in Table 5-1. It is assumed that the reason for the intermediate grid structure producing the most accurate result for the outlet bulk fluid temperature is due to the boundary layer grid spacing being further from the wall in the 496,154 element grid than in the 222,222 element grid. The literature associated with the THTL states that a single thermocouple in the flow field was used to measure this quantity. The uncertainty associated with thermocouple readings, in general, can be as high as $\pm 3^{\circ} \mathrm{C}$. The uncertainty in the measurement, however, was not recorded in the data set.

The accuracy of the inlet pressure improved as the grid structure became finer. The relative error associated with the 496,154 element structure was calculated to be $0.297 \%$. This is a marked improvement over the coarser structures where the errors are $6.87 \%$ and $7.46 \%$ for the 82,200 element structure and the 222,222 element structure, respectively. It should also be pointed out that the finer structure produces an inlet pressure magnitude higher than the measured value whereas the coarser structures produce inlet pressure magnitudes less than the measured value.

The test section power, i.e. TS Power, exhibits a very low error for all three mesh cases, the error decreasing as the grid structure becomes finer. The final value of the relative error of 
the TS Power is calculated to be $0.149 \%$. The magnitude of the test section power was evaluated by integrating the power density over four times the model volume, i.e. total geometric volume.

TS Power $r_{\text {model }}=4 * \int q^{\prime \prime \prime} d v$

The volume is an extensive property. Therefore, to obtain a total value of the power generated in the system, one must integrate over the entire material volume.

The maximum heat flux magnitude, measured at the interface between the solid and the fluid, also approached the measured value as the grid structure was refined. The relative error associated with the finest grid structure was $0.059 \%$. Interestingly, the relative error in the average heat flux magnitude at the interface between the fluid and the solid is minimized, with respect to the other grid structures, using the intermediate grid structure. The minimum error calculated is $1.069 \%$. The relative error associated with the fine grid structure, i.e. 496,154 elements, is $1.575 \%$.

The average velocity at a cross section was calculated by evaluating the mass flow rate through the system then dividing out the mass density and the cross sectional area, i.e.

$\bar{V}=\frac{\dot{m}}{\rho A_{c}}$

The average velocity at the entrance of the computational domain was calculated using Equation 5.8 with the mass density evaluated at the entrance temperature. The average velocity at the exit was calculated using Equation 5.8 with the mass density evaluated at the exit temperature. These values were added then divided by 2 to give the average velocity through the system. The average velocity through the system follows the same pattern where the intermediate grid structure provides the minimum relative error. The minimum relative error for the average velocity is $1.409 \%$.

\subsection{Grid Convergence Index}

The Grid Convergence Method (GCM) provides an accepted and recommended method for the calculation of discretization error associated with numerical computation [37]. The representative grid size is calculated by 
$G S=\left(\frac{V_{\text {grid }}}{n}\right)^{1 / 3}$

Here $G S$ is the grid size, $V_{\text {grid }}$ is the volume occupied by the grid structure, and $n$ is the total number of elements within $V_{\text {grid }}$. Using Equation 5.9 the representative grid sizes of the three grids under consideration for the THTL are $1.28 \times 10^{-10}, 4.739 \times 10^{-11}, 2.122 \times 10^{-11}$ from coarsest to finest. The grid refinement factor is

$r=\frac{G S_{\text {coarse }}}{G S_{\text {fine }}}$

It is recommended that $r>1.3$. The values of $r$ for the grid structures considered here are 2.7009 and 2.2327 for the ratios of coarse grid to intermediate grid and intermediate grid to fine grid, respectively. In what follows, $\psi$, will represent any one of the experimentally determined quantities shown in Table 5-1. The apparent order of the method, $M$, is evaluated using

$M=\frac{1}{r_{i f}}|\ln | \frac{\psi_{c}-\psi_{i}}{\psi_{i}-\psi_{f}}\left|+\ln \left[\frac{r_{i f}^{M}-1 \cdot \operatorname{sgn}\left(\frac{\psi_{c}-\psi_{i}}{\psi_{i}-\psi_{f}}\right)}{r_{c i}^{M}-1 \cdot \operatorname{sgn}\left(\frac{\psi_{c}-\psi_{i}}{\psi_{i}-\psi_{f}}\right)}\right]\right| ; \quad \operatorname{sgn}(x)=\left\{\begin{array}{c}-1 \text { for } x<0 \\ 0 \text { for } x=0 \\ 1 \text { for } x>0\end{array}\right.$

Here the subscript $c$ stands for coarse grid, the subscript $i$ stands for intermediate grid, and $f$ stands for fine grid, therefore, $r_{i f}=\frac{G S_{\text {intermediate }}}{G S_{\text {fine }}}$. One notices that equation 5.11 is a transcendental equation for $M$, this calculation is done in an iterative process. A good initial guess with which to begin the iteration process is

$M_{0}=\frac{1}{r_{i f}}|\ln | \frac{\psi_{c}-\psi_{i}}{\psi_{i}-\psi_{f}}||$ 
The Grid Convergence Index, GCI, is then determined by

$G C I=\frac{1.25 e_{r e l}}{r_{i f}^{M}-1}$

Here $e_{r e l}$ is the relative error in quantity of interest, i.e.

$e_{r e l}=\left|\frac{\psi_{i}-\psi_{f}}{\psi_{f}}\right|$

The GCI provides a quantitative measure of the uncertainty associated with discretization error. The results of the fine grid values of the quantities presented in Table 5-1 with the discretization error is presented in Table 5-2.

Table 5-2. Fine Grid Structure with Discretization Error Compared with Experimental Data

\begin{tabular}{|l|r|r|}
\hline Quantity & Experiment & $\underline{\mathbf{n}=\mathbf{4 9 6 , 1 5 4}}$ \\
\hline Tout $\left({ }^{\circ} \mathrm{C}\right)$ & 163.09 & $165.021 \pm 0.34 \%$ \\
\hline Pin $(\mathrm{MPa})$ & 2.693 & $2.701 \pm 1.34 \%$ \\
\hline TS Power $(\mathrm{W})$ & 173459 & $173717 \pm 0.12 \%$ \\
\hline Max q" $\left(\mathrm{MW} / \mathrm{m}^{2}\right)$ & 13.514 & $13.506 \pm 0.93 \%$ \\
\hline Ave q" $\left(\mathrm{MW} / \mathrm{m}^{2}\right)$ & 11.875 & $11.688 \pm 0.37 \%$ \\
\hline Ave v $(\mathrm{m} / \mathrm{s})$ & 21 & $20.678 \pm 0.08 \%$ \\
\hline
\end{tabular}

The data for the inlet pressure are within the uncertainty associated with the domain discretization. The remainder of the quantities lie outside the discretization error. It is unfortunate that the experimental error associated with the physical measurements were not reported to better determine the fit of the computational results. However, the relative errors for the fine grid structure reported in Table 5-1 indicate that the model reproduces the experimentally measured results fairly well. 


\section{Chapter 6 3D Model Development: Thermal Hydraulic Characteristics of Fuel Defects Produced During the Manufacturing Process}

Defects in nuclear fuel occur both during device operation as a result of irradiation damage and during the manufacturing process. This work does not consider the transient development of the defects due to irradiation during device operation, but instead considers the global steady state response of the system to the existence of defects which occurred during fuel manufacturing.

As discussed in Chapter 2, previous investigations were based on 2D analysis and numerical simulations. This previous work only concentrated on the local response of the system to a local defect [13] [17]. A constant convection coefficient boundary condition in conjunction with a variable bulk fluid temperature was used in lieu of the turbulent flow field. This limits the system response in that the ratio of surface heat flux in a neighborhood of the defect location to the temperature difference in the same neighborhood is always constant.

The defects to be analyzed are a lack of metallurgical bond between the fuel and the clad, i.e. a blister defect, i.e. regions in the fuel where the local loading of ${ }^{235} \mathrm{U}$ is large relative to nominal values. The upper limit of the fuel segregation for pre-irradiated fuel is $30 \%$ in an area of diameter 5/64 inch [14] , anything larger will be identified by the inspection process which will result in the rejection of the plate. The upper limit for the blister defect is a diameter of 1/16 inch in the pre-irradiated fuel, any larger blister defects result in the rejection of the plate [14].

\subsection{Plate Geometry and Grid Structure}

The HEU model geometry is that of a flat plate with an internal structure composed of the contoured fuel meat, i.e. $\mathrm{U}_{3} \mathrm{O}_{8}$ - $\mathrm{Al} 1100$ cermet. The flat fuel plate resides between two one-half flow channels, i.e. the fluid domain completely surrounds the fuel plate. Figure 6-1 shows a cross-sectional view of the model geometry looking in the flow direction. The fuel plate is 24 inches $(0.6096 \mathrm{~m})$ long. The fluid domain extends 39.37 inches $(1 \mathrm{~m})$ in the axial direction. The fluid 


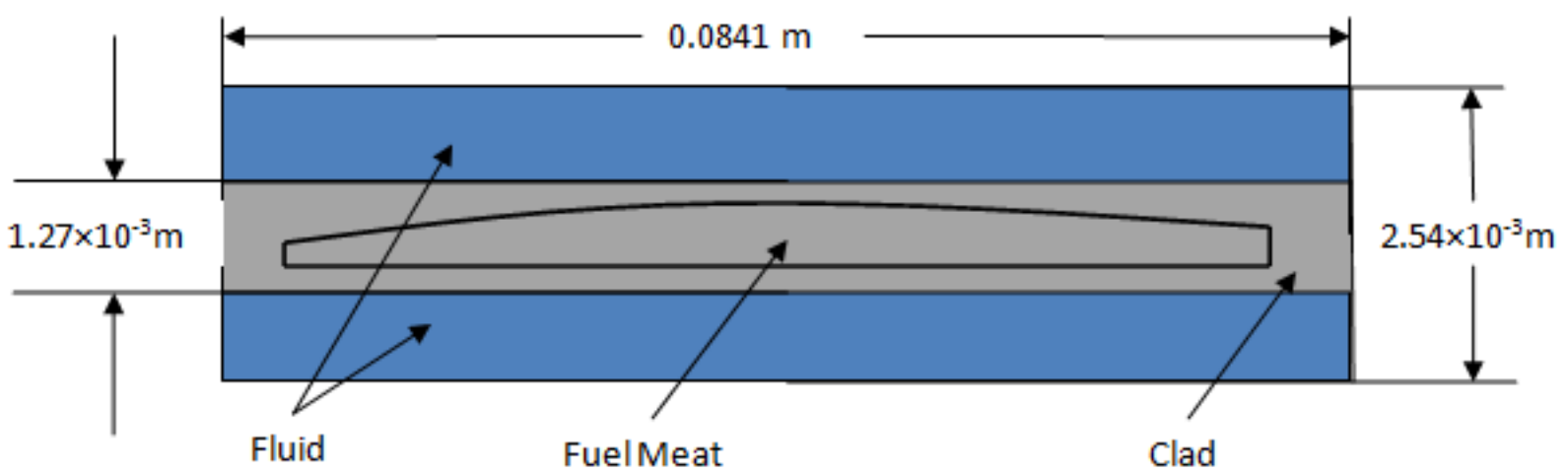

Figure 6-1. Cross-sectional view of the fuel defect model geometry. The vantage is in the flow direction. The dimensions are given for reference. The model geometry is $1 \mathrm{~m}$ long in the flow direction. The contoured side of the fuel meat domain is obvious and will be referenced throughout the chapter. 
domain extends 3.58 inches $(0.091 \mathrm{~m})$ upstream of the fuel plate and 11.81 inches $(0.3 \mathrm{~m})$ downstream of the fuel plate. Again, these extended regions upstream and downstream of the fuel plate are to allow the simulated flow to adjust to the fuel plate obstruction. The contour of the fuel meat was constructed from the detailed drawing of the HEU inner fuel plate [38].

The global boundary condition of the upstream fluid domain is set with a uniform inlet velocity of $7.925 \mathrm{~m} / \mathrm{s}$ and an isothermal value of $327 \mathrm{~K}$. The global boundary condition on the downstream fluid domain is set to a uniform pressure of $2.606 \mathrm{MPa}$. The fluid boundaries on the lateral measure, i.e. $0.0841 \mathrm{~m}$ length in Figure 6-1, are set to thermal and fluid dynamic symmetry conditions. The boundaries on the thickness measure, i.e. $2.54 \times 10^{-3} \mathrm{~m}$ length in Figure 6-1, are adiabatic walls.

The power density used in fuel meat domain is shown in Figure 6-2. Figure 6-2 is a graphical representation of the power density distribution array shown numerically in Figure 2-2. The magnitude of this power density distribution array was adjusted to be consistent with the HFIR power density. The adjustment calculation is based on the total power generated in the HFIR divided by the total volume of fuel meat in the core, i.e.

$q^{\prime \prime \prime}=\frac{Q}{V_{\text {total }}}=\frac{Q}{V_{\text {inner }}+V_{\text {outer }}}$.

Here, as before, $Q$ is the total core power, i.e. $85 \mathrm{MW}$. The total volume of the fuel meat in the core, $V_{\text {total }}$, is the sum of the inner core fuel loading and the outer core fuel loading. These values are $V_{\text {inner }}=3.64 \times 10^{-3} \mathrm{~m}^{3}$ for the inner core and $V_{\text {outer }}=6.39 \times 10^{-3} \mathrm{~m}^{3}$ for the outer core. These fuel volumes were obtained using cubic spline interpolation of the HFIR plate fuel meat contour points [38] in conjunction with Simpson's quadrature rule as outlined in Appendix B. Equation 6.1 may now be evaluated and it is found that the total power density of the HFIR core is $8.47 \times 10^{9} \mathrm{~W} / \mathrm{m}^{3}$. This value is multiplied by the power density distribution function which then adjusts the magnitude of the array. However, the resultant power generated by the plate is inconsistent with the amount of energy generated by a single inner fuel plate and in fact is $15.3 \%$ too large. The power generated by a single inner fuel plate is 


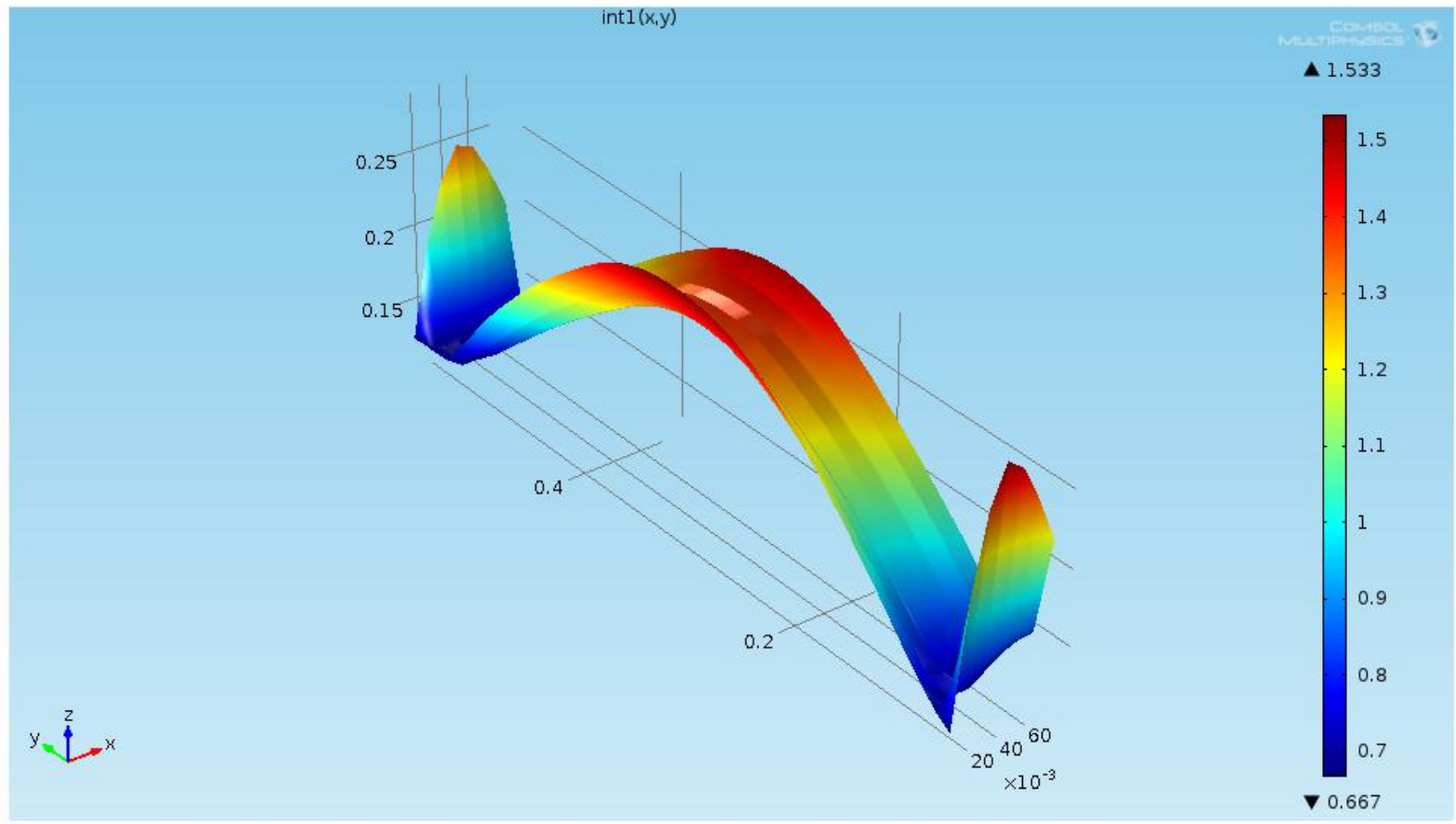

Figure 6-2. The 3-D Power Density Profile used in the Fuel Meat Domain of the Simulation Geometry. 
$q_{\text {inner }}=\frac{q^{\prime \prime \prime} V_{\text {inner }}}{171}$

Equation 6.2 yields a value of $q_{\text {inner }}=1.80 \times 10^{5} \mathrm{~W}$. This $15.3 \%$ discrepancy is due to the power density distribution array. The power density distribution array is not a unit normal distribution. Therefore, the sum of all the values in the array does not equal unity, but instead results in a value larger than unity. As a result, only $84.6 \%$ of the power density magnitude, i.e. $7.17 \times 10^{9} \mathrm{~W} / \mathrm{m}^{3}$, is distributed by the array and produces energy consistent with the $1.80 \times 10^{5} \mathrm{~W}$ generated per inner fuel plate. This value was calculated by executing the simulation with the generation rate $8.47 \times 10^{9} \mathrm{~W} / \mathrm{m}^{3}$, then evaluating the volume integral of the generation rate in the fuel meat from the results.

The turbulence equation set used for the fuel defect analysis was Wilcox's k- $\omega$ closure model as described in Section 3.2.1. The complexity of the geometry made a grid structure suitable for the Low Reynolds Number k- $\varepsilon$ model prohibitively large regarding memory requirements.

The grid structure is established using a mixture of tetrahedral elements and boundary layer elements, i.e. rectangular prisms. The tetrahedral grid structure in the fluid domain has a maximum element size of $4.13 \times 10^{-3} \mathrm{~m}$, a minimum element size of $2.51 \times 10^{-4} \mathrm{~m}$, and a maximum growth rate of 1.25. The tetrahedral grid structure in the clad and fuel domains have a maximum element size of $0.19 \mathrm{~m}$, a minimum element size of $2.51 \times 10^{-4} \mathrm{~m}$, and a maximum growth rate of 1.7. The boundary layer grid structure, i.e. those elements closest to the solid domain extending into the fluid, has a maximum element size of $2.13 \times 10^{-3} \mathrm{~m}$, a minimum element size of $6.35 \times 10^{-4} \mathrm{~m}$, and a maximum element growth rate of 1.05 . The boundary layer grid structure has 10 elements with a boundary layer stretching factor of 1.1. Given the dimensionality and the large aspect ratio of the model geometry, the author has concluded that images of the grid structure would serve no purpose other than to confuse the reader. However, the details listed above are adequate for recreation of the grid structure within the COMSOL environment.

The solution method uses a segregated solver with a maximum iteration number of 400 . The tolerance factor was set to unity with a relative tolerance of $1 \times 10^{-6}$. 
The first segregation encompasses the velocity, the pressure, and the temperature. The Newton iterations use a constant damping factor of 0.5 and the Jacobian is updated on every iteration. The Generalized Minimum RESidual (GMRES) method is used to solve the system of equations. Preconditioning of the system matrix is carried out using the Successive Over Relaxation (SOR) iterative method with the velocity as the line variable. The maximum line length is set to 5 with a relaxation factor of 0.5 .

The second segregation encompasses the turbulence variables, i.e. the specific turbulent kinetic energy and the dissipation rate of the turbulent kinetic energy. The Newton iterations, for the second segregation, use a constant damping factor of 0.3 and the Jacobian is updated on every iteration. Again the GMRES algorithm is used to solve the system of equations with the SOR algorithm preconditioning the system matrix. The turbulent kinetic energy is used as the line variable for this segregation with a maximum line length set to 5 with a relaxation factor of 0.5 .

Three different models will be discussed in this section. The nominal fuel plate case will be used to establish a base line with which to compare the blister defect model and the fuel segregation model.

\subsection{Nominal Plate Case}

The nominal plate case is an idealization where the fuel is free of any defects. To expand on this definition of idealism, the fuel, the clad, and the fluid are in perfect thermal contact and the fuel meat is homogenous throughout the fuel meat domain.

The temperature distribution on the surface of the clad material for the nominal plate case is shown in Figure 6-3 with the maximum and minimum surface temperature locations superposed on the Figure. The maximum temperature is found to be $211.72^{\circ} \mathrm{F}(372.99 \mathrm{~K})$ which occurs on the uncontoured, i.e. side of the fuel plate where the fuel meat is not contoured, side of the fuel plate at a location just slightly upstream of the fuel meat-clad interface, i.e. $\mathrm{z}=0.0508$ $\mathrm{m}$, on the lower end of the fuel plate, i.e. 0.1004 in $\left(2.55 \times 10^{3} \mathrm{~m}\right)$ in the positive $\mathrm{z}$ direction above 


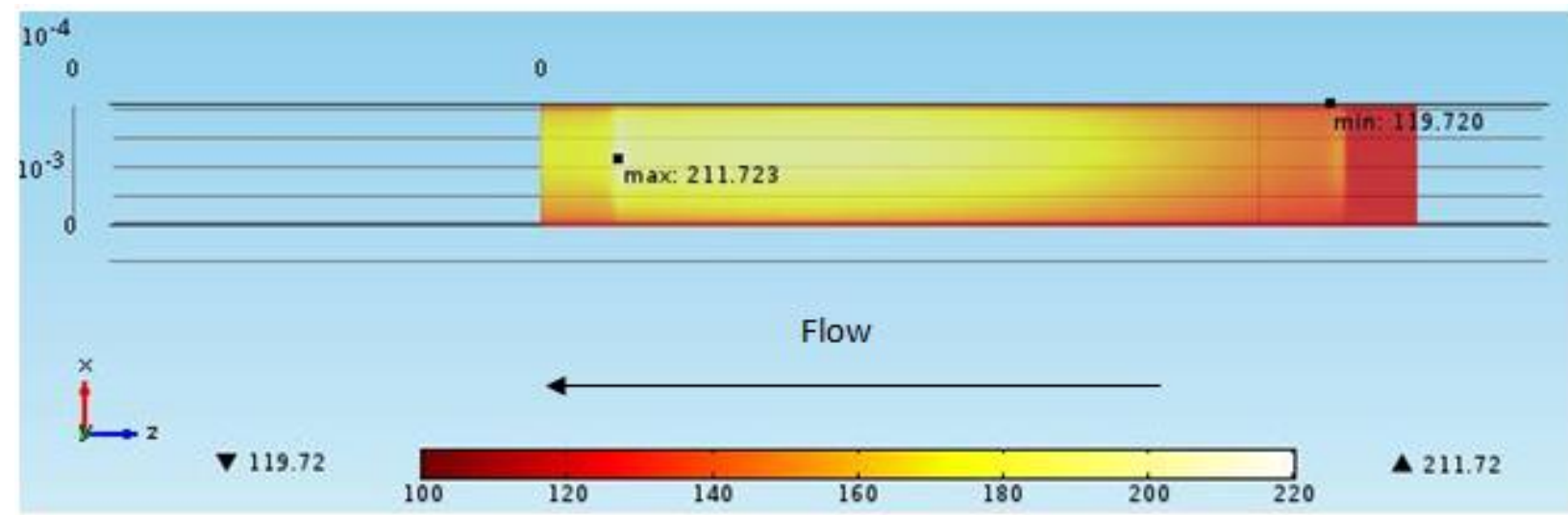

Figure 6-3. Nominal Case Clad Surface Temperature Along the Uncontoured Side of the Fuel Plate. The maximum and minimum surface temperatures are superposed on the image at the location of their occurrence. 
the interface. The spatial location of the maximum surface temperature is $\mathrm{x}=0.04593 \mathrm{~m}, \mathrm{y}=0$ $\mathrm{m}$, and $\mathrm{z}=0.05335 \mathrm{~m}$. As stated earlier in Chapter 2, the saturation temperature range is from $463.33^{\circ} \mathrm{F}$ to $438.88^{\circ} \mathrm{F}$, i.e. from the leading edge of the fuel plate to the trailing edge, respectively. The saturation temperature and pressure at the location of the maximum temperature on the uncontoured side of the fuel plate is calculated to be $439.29^{\circ} \mathrm{F}(499.42 \mathrm{~K})$ and 379.3 psig $\left(2.615 \times 10^{6} \mathrm{~Pa}\right)$, respectively. Therefore, induced two-phase flow is not a concern for the nominal plate case on the uncontoured side. Since the maximum temperature for the nominal case occurred on the uncontoured side, it stands to reason that the contoured side of the fuel plate does not pose a potential two-phase flow threat. Indeed, the maximum temperature occurring on the contoured side of the nominal fuel plate was calculated to be $209.89^{\circ} \mathrm{F}(371.98 \mathrm{~K})$ at an equivalent location for $\mathrm{x}$ and $\mathrm{z}$, but at $\mathrm{y}=0.00127 \mathrm{~m}$.

The absolute maximum heat flux from the plate to the fluid occurs on the uncontoured side of the fuel plate with a magnitude of $3.331 \times 10^{6} \mathrm{~W} / \mathrm{m}^{2}$ at the lateral and axial center, i.e. $\mathrm{x}=$ $0.04483 \mathrm{~m}, \mathrm{y}=0 \mathrm{~m}$, and $\mathrm{z}=0.31057 \mathrm{~m}$. The surface heat flux is shown in Figure 6-4. The heat flux occurring at the maximum temperature location was calculated to be $9.586 \times 10^{5} \mathrm{BTU} / \mathrm{hr} / \mathrm{ft}^{2}$ $\left(3.024 \times 10^{6} \mathrm{~W} / \mathrm{m}^{2}\right)$, which represents a $9.3 \%$ decrease in heat flux from the maximum. It will be shown that the blister defect has a similar characteristic, i.e. that the maximum heat flux does not occur at the defect, however, the segregation defect does indeed shift the location of the maximum heat flux to the defect location. The minimum heat flux shown in Figure 6-4 has a negative magnitude, due to the reversal of the heat flux in the unfueled portion of the fuel plate. This reversal of heat flux is due to the bulk fluid temperature being larger in magnitude than the clad surface temperature in the unfueled region of the fuel plate, i.e. heat is passed from the fluid back into the fuel plate in this region. It is important to note that the incipient boiling heat flux calculated by the Bergles and Rohsenow correlation will result in a negative value due to the wall temperature, $T_{w}$, being lower in magnitude than the saturation temperature, $T_{\text {sat }}$. The Bergles and Rohsenow correlation [39] follows:

$q_{i b}^{\prime \prime}=15.6 P^{1.156}\left(T_{w}-T_{s a t}\right)^{\frac{2.3}{P^{0.0234}}}$

Here $q_{i b}^{\prime \prime}$ is the incipient boiling heat flux and $P$ is the pressure. 


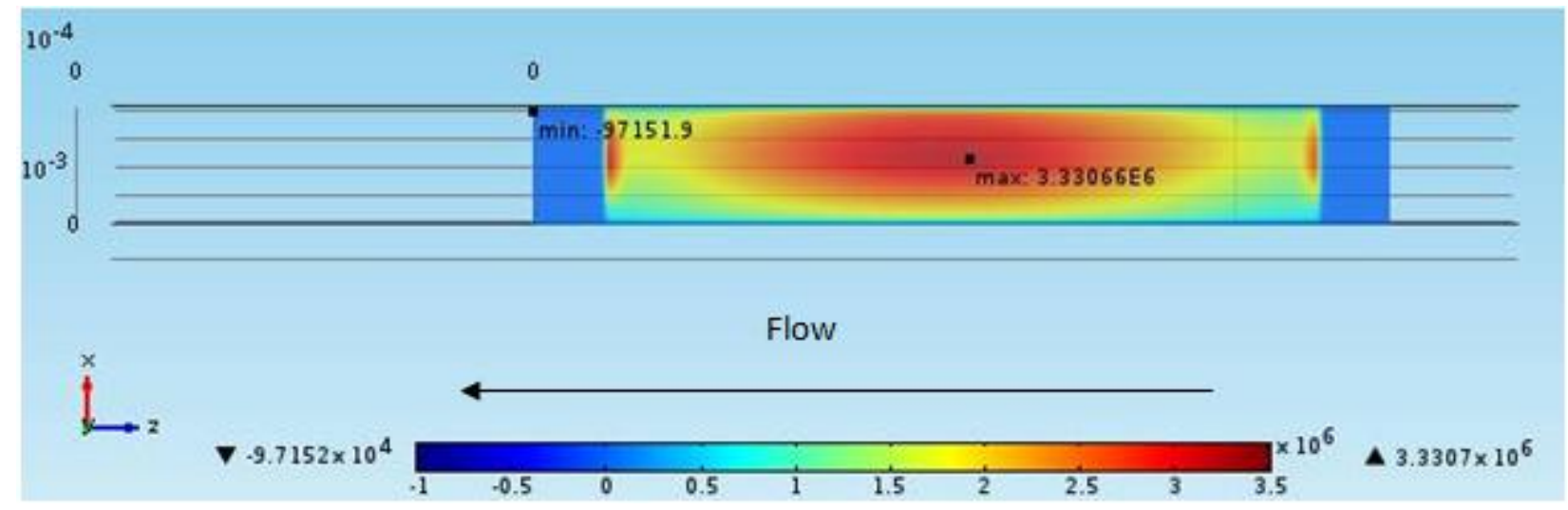

Figure 6-4. Nominal Case Clad Surface Heat Flux Along the Uncontoured Side of the Fuel Plate. The maximum and minimum heat flux values are superposed on the image at the location of their occurrence. 
The convection coefficient will be defined using the inlet bulk fluid temperature, $T_{b_{i n}}$, as the reference temperature, which is established as a global boundary condition for the model and has a magnitude of $120.00^{\circ} \mathrm{F}(322.04 \mathrm{~K})$, i.e.

$h:=\frac{q^{\prime \prime}}{\left(T_{s}-T_{b_{i n}}\right)}$.

The choice to use the inlet bulk fluid temperature as the reference temperature is one of convenience. This choice removes the cumbersome evaluation of the bulk fluid temperature at various locations along the fuel plate. In many ways, this is a more practical measure of the convection coefficient as the inlet bulk temperature is easier to determine experimentally than the downstream bulk fluid temperature at various locations where thermal energy is being exchanged. Using definition 6.4, the magnitude of the convection coefficient at the location of the maximum clad surface temperature is found to be $1.044 \times 10^{4} \mathrm{BTU} / \mathrm{hr} / \mathrm{ft}^{2} /{ }^{\circ} \mathrm{F}\left(5.927 \times 10^{4}\right.$ $\mathrm{W} / \mathrm{m}^{2} / \mathrm{K}$ ). This result provides a more conservative estimate of the convection coefficient than that used in previous analyses, i.e. $1.5 \times 10^{4} \mathrm{BTU} / \mathrm{hr} / \mathrm{ft}^{2} /{ }^{\circ} \mathrm{F}\left(8.5 \times 10^{4} \mathrm{~W} / \mathrm{m}^{2} / \mathrm{K}\right)$. The convection coefficient evaluated using Equation 6.4 produces a value lower in magnitude than the value used in previous analyses, therefore, allowing less thermal energy to pass from the plate to the fluid thus resulting in higher plate surface temperatures.

The pressure drop through the nominal case system is calculated to be $86.7 \mathrm{psig}$ $\left(5.98 \times 10^{5} \mathrm{~Pa}\right)$. This deficiency in pressure drop, i.e. from the measured $103 \mathrm{psi}$ drop through the core, can be partially attributed to the idealized surface condition associated with the computational model, i.e. the clad surface is mathematically smooth. Another cause of the pressure drop deficiency could be due to grid resolution. The error in the conservation of energy for the computational model of the nominal plate is calculated to be only $3.83 \%$, i.e. the net efflux of thermal energy minus the thermal energy passed from the fuel plate to the fluid normalized by the integrated plate-to-fluid heat flux.

$E_{\text {energy }}=\left\{\frac{\left[\left(\int \rho C_{p} U T d A_{\text {outlet }}\right)-\left(\int \rho C_{p} U T d A_{\text {inlet }}\right)\right]-\oint q^{\prime \prime} d A_{\text {clad }}}{\oint q^{\prime \prime} d A_{\text {clad }}}\right\} 100$ 
The error in the conservation of mass was calculated to be $4.77 \times 10^{-3} \%$. This quantity was calculated by the ratio of the net areal integrated mass flux into the system normalized by the inlet areal integrated mass flux.

Knowledge of the position at which the maximum clad surface temperature occurs allows one to determine the most unfavorable location for a defect, i.e. unfavorable in the sense of increasing surface temperatures further. Since the defect acts either as a region of lack of thermal contact, i.e. blister defect, or as a region of excessive local thermal energy generation, i.e. segregation defect, one can summarize the effect as a region which causes a local increase in the clad surface temperature. With this in mind, it stands to reason that the placement of a fuel defect at the location where the clad surface temperature has its maximum value in the nominal case will have the effect of further increasing the clad surface temperature at that location. As a result, the defects to be investigated in this work will be created at the axial location of the maximum clad surface temperature found for the nominal case.

\subsection{Blister Defect Case}

As previously stated, a blister is a region where the metallurgical bond between the clad material and the fuel meat has deteriorated, i.e. no thermal contact between the clad and fuel exists at the location of the defect. The geometry of the blister defect and its relative location to the fuel and clad material is shown in Figure 6-5 for visualization purposes only. The blister defect exists at the interface between the contoured side of the fuel and the clad. The blister defect case is similar to this The blister defect was introduced into the nominal case model by the creation of an adiabatic disc 1/16 inch in diameter which follows the contour of the fuel meat and lies at the interface between the fuel meat and the clad at the location of the maximum temperature occurring in the nominal case. The 1/16 inch diameter disk is consistent with the rejection criteria used in the inspection of the fuel plate after fabrication. A significant effort was required 


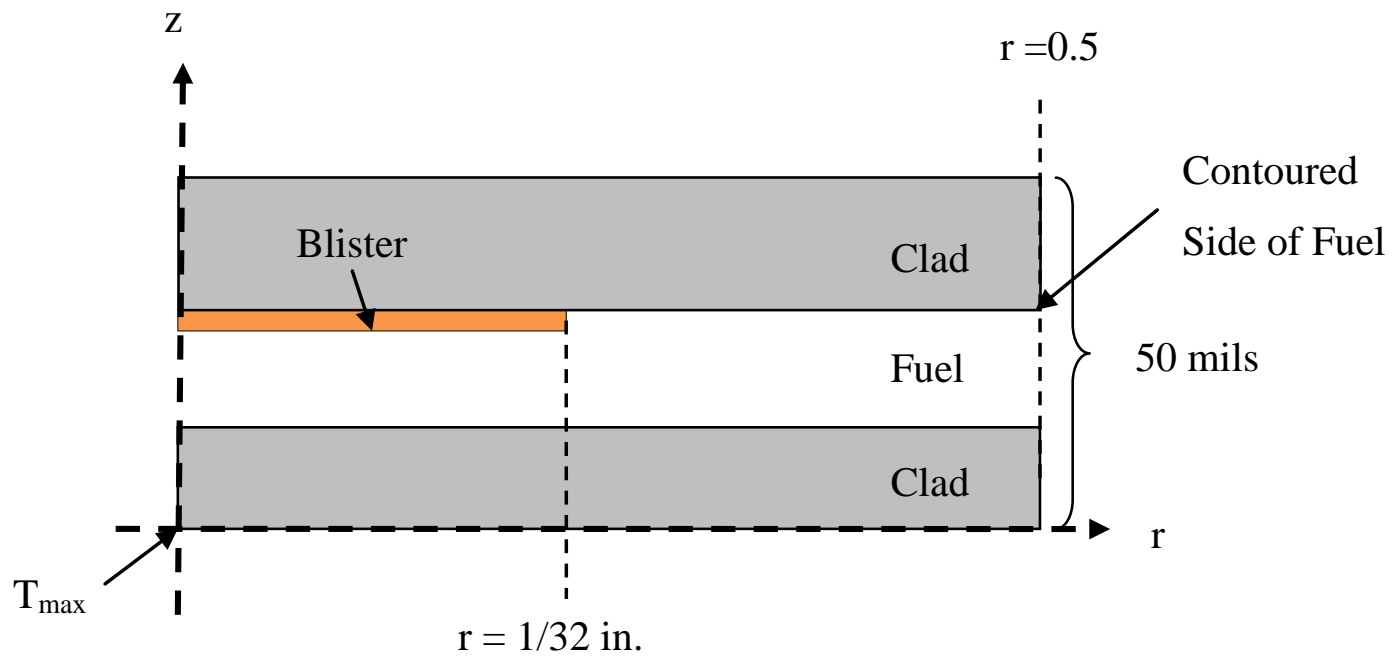

Figure 6-5. Blister Defect Geometry Relative to the Surrounding Fuel and Clad. This model does not consider segregation defects. The location of the maximum clad surface temperature, $\mathrm{T}_{\max }$, from the nominal case is indicated. 
to create the blister defect region within the computational environment. The adiabatic nature of the interfacial disk sufficiently represents the lack of thermal contact between the fuel meat and the clad that occurs in the physical set-up. It is important to note that with the exception of the blister defect all domains of the model are in perfect thermal contact.

The maximum temperature associated with the blister defect case again occurs on the uncontoured side of the fuel plate and has a magnitude of $212.92^{\circ} \mathrm{F}(373.66 \mathrm{~K})$, which is located at $\mathrm{x}=0.0478 \mathrm{~m}, \mathrm{y}=0 \mathrm{~m}, \mathrm{z}=0.05398 \mathrm{~m}$, as shown in Figure 6-6. The slight shift in the location of the maximum temperature relative to the nominal case is likely due to the slight adjustment in the grid structure necessary to incorporate the blister defect. The pressure of the fluid at the defect location is $379.56 \mathrm{psig}\left(2.617 \times 10^{6} \mathrm{~Pa}\right)$, which yields a saturation temperature of $439.34^{\circ} \mathrm{F}$ (499.45 K). Again, the blister defect does not produce sufficient surface temperatures to transition to two-phase flow.

The absolute maximum heat flux for the blister defect case, shown in Figure 6-7, occurs on the uncontoured side of the fuel plate as well and has a magnitude of $1.057 \times 10^{6} \mathrm{BTU} / \mathrm{hr} / \mathrm{ft}^{2}$ $\left(3.335 \times 10^{6} \mathrm{~W} / \mathrm{m}^{2}\right)$, which represents a $0.12 \%$ increase from the nominal case. As previously stated, the maximum heat flux occurs near the center of the clad surface and not at the defect location. The absolute maximum heat flux is located at $\mathrm{x}=0.0456 \mathrm{~m}, \mathrm{y}=0 \mathrm{~m}, \mathrm{z}=0.3093 \mathrm{~m}$. The heat flux at the defect location was found to be $9.824 \times 10^{5} \mathrm{BTU} / \mathrm{hr} / \mathrm{ft}^{2}\left(3.099 \times 10^{6} \mathrm{~W} / \mathrm{m}^{2}\right)$. The heat flux at the defect location has increased by $2.48 \%$ over the nominal case at essentially the same location. Again, the minimum heat flux is represented by a negative quantity over the downstream, unfueled, region of the clad which implies a reversal of heat flux. The defect acts to decrease the available heat transfer area of the plate. Decreasing the available heat transfer area while maintaining the thermal generation rate, increases the overall heat flux from the plate. The largest increase occurring at the location of the defect with a diminishing influence through the rest of the plate. This is observed in comparing the nominal and blister cases. As stated, the absolute maximum surface heat flux, which occurs $0.26 \mathrm{~m}$ upstream of the blister defect, increased slightly by $0.12 \%$. However, the surface heat flux at the blister defect location increased by $2.48 \%$. The convection coefficient at the defect location, defined in Equation 6.4, 


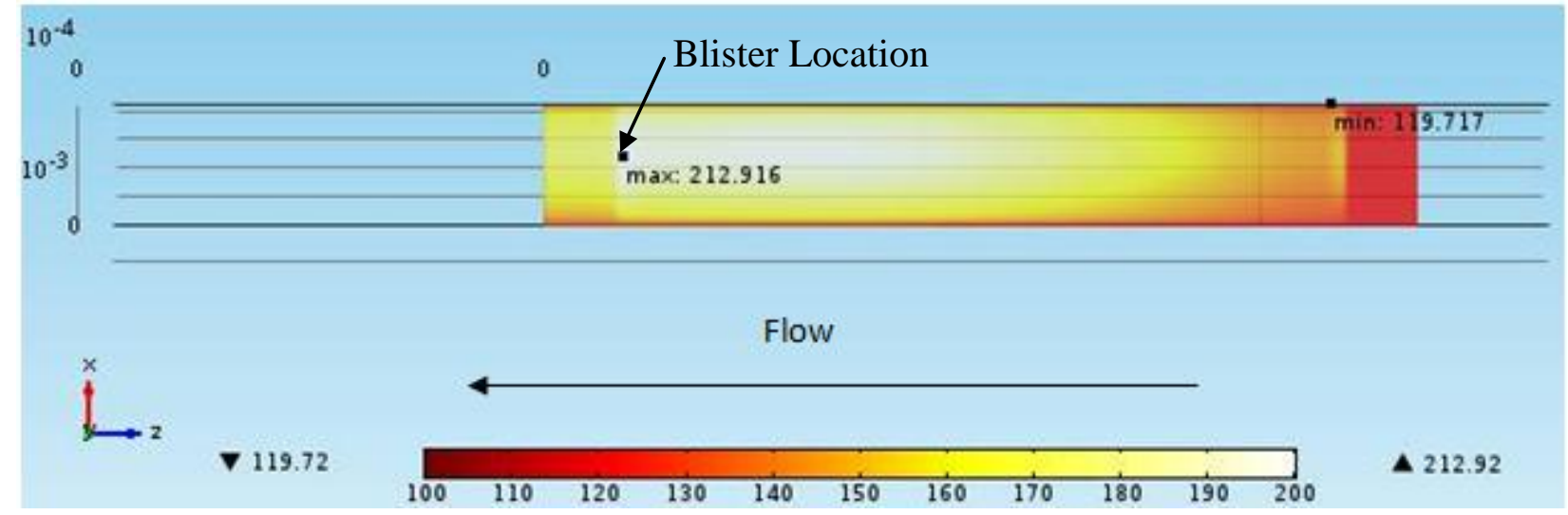

Figure 6-6. Blister Case Clad Surface Temperature Along the Uncontoured Side of the Fuel Plate. Maximum and minimum temperatures are superposed on the image at the locations of their occurrence. 


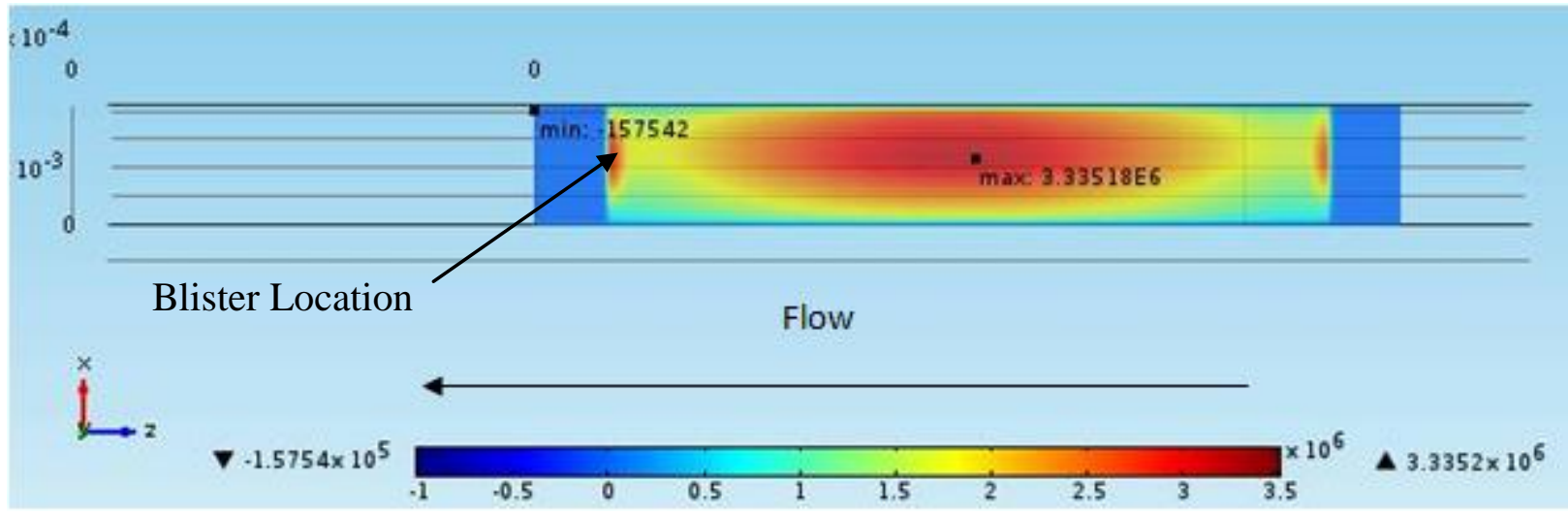

Figure 6-7. Blister Case Clad Surface Heat Flux Along the Uncontoured Side of the Fuel Plate. The maximum and minimum values of the heat flux are superposed on the image at the location of their occurrence. 
yields a value of $1.06 \times 10^{4} \mathrm{BTU} / \mathrm{hr} / \mathrm{ft}^{2} /{ }^{\circ} \mathrm{F}\left(6.00 \times 10^{4} \mathrm{~W} / \mathrm{m}^{2} / \mathrm{K}\right)$, which is only a $1.18 \%$ increase over the nominal case at the same location.

The pressure drop through the blister defect model was calculated to be $86.9 \mathrm{psig}$ $\left(5.99 \times 10^{5} \mathrm{~Pa}\right)$. The error in the global energy balance was calculated using Equation 6.5 and was found to have a magnitude of $4.03 \%$. The error in the global balance of mass was calculated to be $8.34 \times 10^{-3} \%$.

It was shown that the blister defect caused an overall increase in the surface heat flux of the fuel plate. This overall increase is maximized at the defect location and dissipates as one moves away from the defect location. The absolute maximum surface heat flux remained at the center of the uncontoured side of the fuel plate surface. The increase in surface heat flux caused an increase in surface temperature with the maximum temperature occurring in approximately the same spot as in the nominal case, however, the maximum was only $0.18 \%$ higher in the blister case. The convection coefficient increased slightly from $5.927 \times 10^{4} \mathrm{~W} / \mathrm{m}^{2} / \mathrm{K}$ in the nominal case to $6.00 \times 10^{4} \mathrm{~W} / \mathrm{m}^{2} / \mathrm{K}$ in the blister case.

\subsection{Segregation Defect Case}

The segregation defect was introduced into the nominal case geometry as a cylinder with a diameter of 5/64 inch, i.e. consistent with the rejection criteria, and extending through the fuel meat domain, i.e. approximately 0.030 inch $\left(7.6 \times 10^{-4} \mathrm{~m}\right)$. The geometry of the segregation defect and its relative location to the fuel and clad material is shown in Figure 6-8 for visualization purposes only. The segregation cylinder was made to conform to the contour of the fuel meat. The segregation defect was positioned at the location of the maximum temperature encountered in the nominal case. As previously stated, the segregation defect is physically characterized by an excess loading of ${ }^{235} \mathrm{U}$. To model this defect the thermal energy generation rate per unit volume within the defect cylinder was increased by an order of magnitude, i.e. $7.17 \times 10^{10} \mathrm{~W} / \mathrm{m}^{3}$. This order of magnitude increase was taken from previous studies of segregation defects [13] [17] [14]. The grid structure used to discretize the segregation defect domain is identical to the 


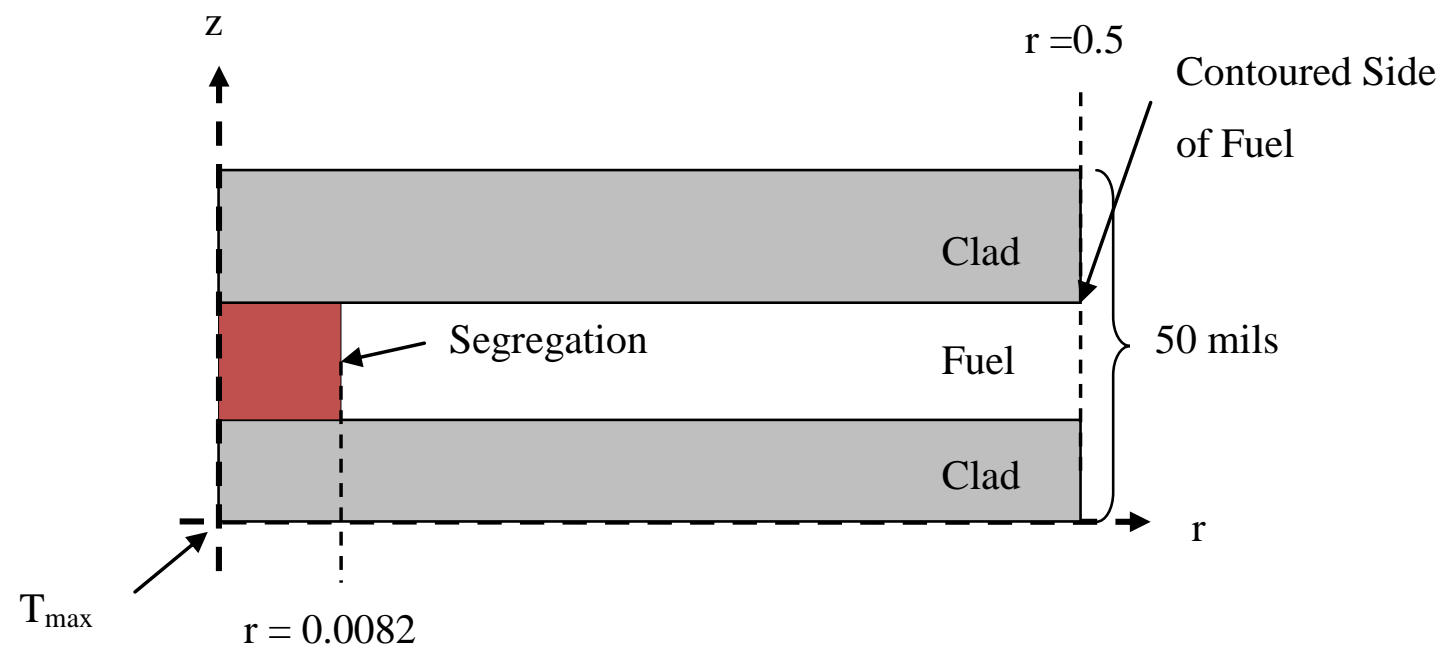

Figure 6-8. Segregation Defect Geometry Relative to the Surrounding Fuel and Clad. This model does not consider blister defects. The location of the maximum clad surface temperature, $\mathrm{T}_{\max }$, from the nominal case is indicated. 
grid structure for the fuel meat domain described earlier. Here the defect and all other domains within the model are assumed to be in perfect thermal contact.

The maximum temperature associated with the segregation defect was found to be located on the fuel contour side of the fuel plate with position coordinates $\mathrm{x}=0.04756 \mathrm{~m}, \mathrm{y}=$ $0.00127 \mathrm{~m}$, and $\mathrm{z}=0.05376 \mathrm{~m}$. The magnitude of the maximum temperature was found to be $219.24^{\circ} \mathrm{F}(377.17 \mathrm{~K})$ as shown in Figure 6-9. The pressure of the fluid at the defect location was calculated to be $380.6 \mathrm{psig}\left(2.624 \times 10^{6} \mathrm{~Pa}\right)$ which yields a saturation temperature of $439.61^{\circ} \mathrm{F}$ (499.60 K). The segregation defect does not produce clad surface temperatures consistent with the saturated state of the fluid therefore the probability of two-phase flow is very low.

The location of the absolute maximum heat flux for the segregation case shifted to the segregation defect location. This is an intuitive result since the thermal energy generation rate per unit volume was increased by an order of magnitude at this location. This is indeed a most unfavorable condition since the bulk fluid temperature is a maximum in the region where the absolute maximum heat flux occurs, i.e. lower fueled region of the fuel plate. However, it has been shown that the probability of two-phase flow is very low. The absolute maximum heat flux magnitude was found to be $1.10 \times 10^{6} \mathrm{BTU} / \mathrm{hr} / \mathrm{ft}^{2}\left(3.48 \times 10^{6} \mathrm{~W} / \mathrm{m}^{2}\right)$. This represents an increase of $4.19 \%$ over the absolute maximum heat flux found in the blister defect case, and a $4.50 \%$ increase over the absolute maximum heat flux found in the nominal case. Since the location of the absolute maximum heat flux has shifted downstream relative to the location found in the other cases, i.e. nominal and blister defect cases, the heat flux at the segregation defect location should be compared with the heat fluxes for the other cases at this location. The segregation defect heat flux is $12.3 \%$ higher than the heat flux at the blister defect location, and it is $15.1 \%$ higher than the heat flux in the nominal case at the same location. The surface heat flux on the fuel contour side of the fuel plate is shown in Figure 6-10. Again, the minimum heat flux occurs at the downstream unfueled region of the fuel plate which, as previously stated, represents a reversal of heat flux, i.e. from the fluid to the fuel plate.

The convection coefficient at the defect location was calculated to be $1.111 \times 10^{4}$ $\mathrm{BTU} / \mathrm{hr} / \mathrm{ft}^{2} /{ }^{\circ} \mathrm{F}\left(6.307 \times 10^{4} \mathrm{~W} / \mathrm{m}^{2} / \mathrm{K}\right)$. This value represents a $5.17 \%$ increase over the convection 


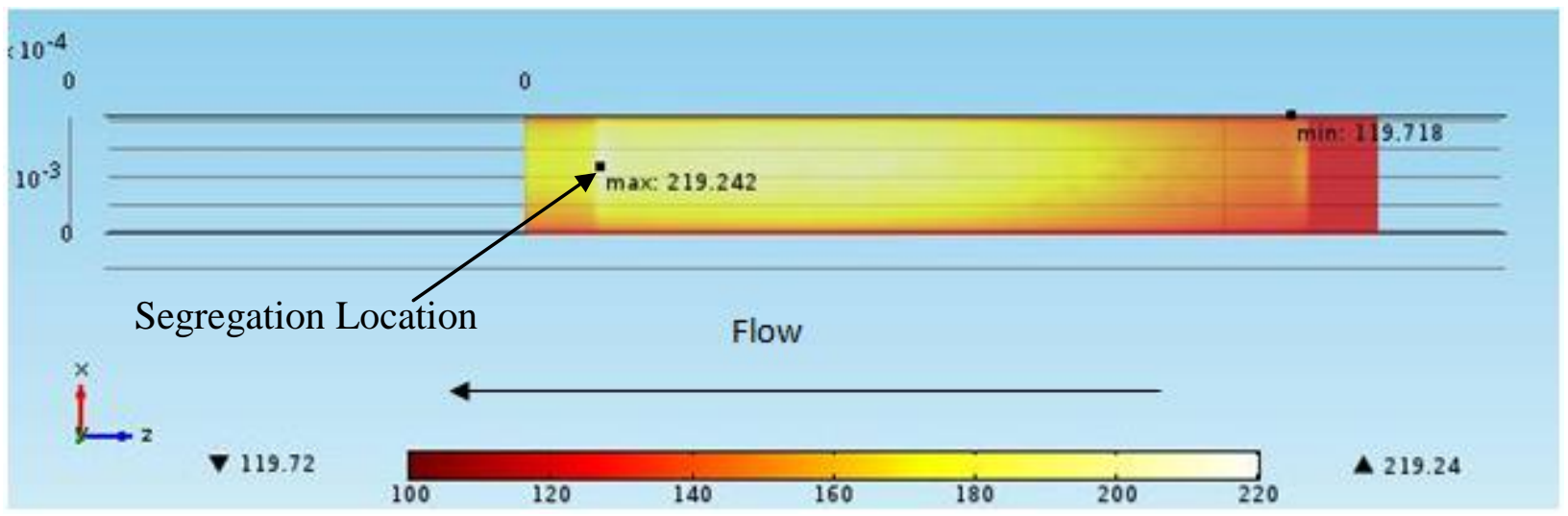

Figure 6-9. Clad Surface Temperature on the Fuel Contour Side of the Fuel Plate. The maximum and minimum temperatures are superposed on the image at the location of their occurrence.

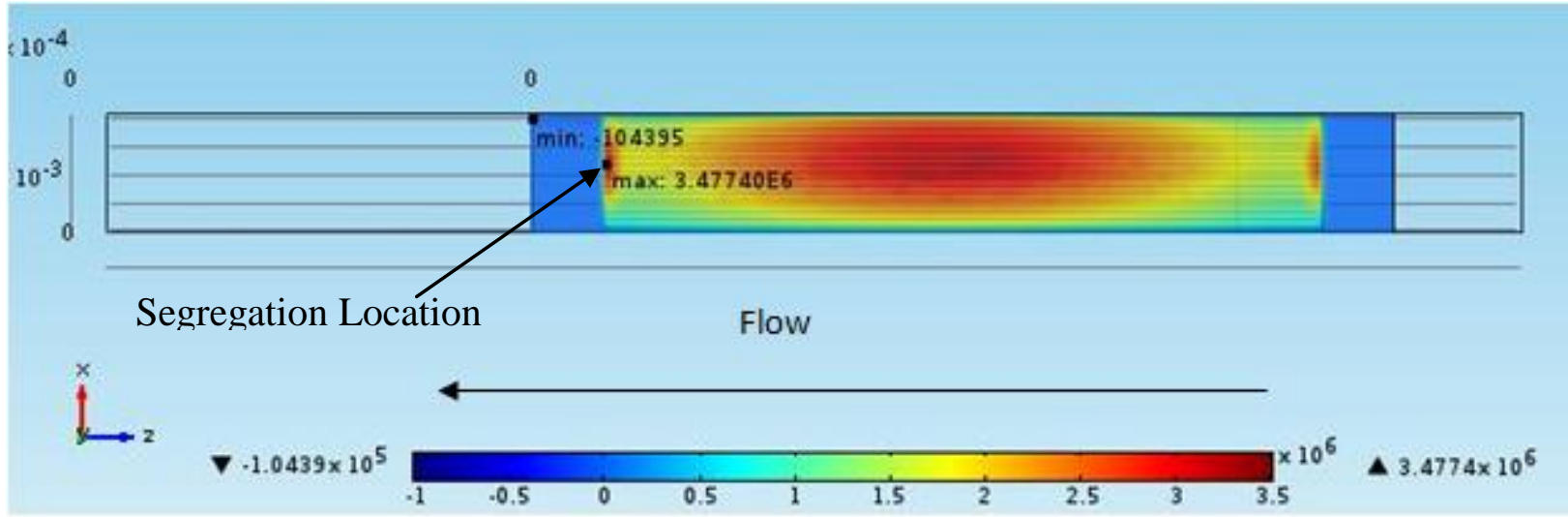

Figure 6-10. Clad Surface Heat Flux on the Fuel Contour Side of the Fuel Plate. The maximum and minimum surface heat flux values have been superposed on the image at the location of their occurrence. 
coefficient at the blister defect location and a $6.46 \%$ increase over the nominal case at the same location.

The pressure drop through the segregation defect system was calculated to be $81.3 \mathrm{psig}$ $\left(5.60 \times 10^{5} \mathrm{~Pa}\right)$. The error in energy conservation was calculated to be $3.83 \%$. The error in mass conservation was calculated to be $5.96 \times 10^{-3} \%$.

It has been shown that of the two defects analyzed, i.e. blister defect and segregation defect, the segregation defect caused the largest changes in the system. This was expected since the thermal generation rate per unit volume was increased in the segregation defect thus increasing thermal output from the plate. The absolute maximum heat flux for the segregation case was found to have shifted position to the defect location instead of the center of the plate as was found in both the blister defect case and the nominal case.

A summary of the results of the fuel defect analysis is presented in Table 6-1. 
Table 6-1. Summary of Results for the Fuel Defect Analysis

\begin{tabular}{|c|c|c|c|}
\hline Quantity & Nominal Case & Blister Case & Segregation Case \\
\hline $\mathrm{T}_{\max }[\mathrm{K}]$ & 372.99 & 373.66 & 377.17 \\
\hline $\mathrm{q}^{\prime \prime}\left[\mathrm{W} / \mathrm{m}^{2}\right]$ at $\mathrm{T}_{\max }$ location & $3.024 \times 10^{6}$ & $3.099 \times 10^{6}$ & $3.48 \times 10^{6}$ \\
\hline $\mathrm{T}_{\text {sat }}[\mathrm{K}]$ at $\mathrm{T}_{\max }$ location & 499.42 & 499.45 & 499.60 \\
\hline $\mathrm{P}[\mathrm{Pa}]$ at $\mathrm{T}_{\max }$ location & $2.615 \times 10^{6}$ & $2.617 \times 10^{6}$ & $2.624 \times 10^{6}$ \\
\hline $\mathrm{q}_{\text {max }}\left[\mathrm{W} / \mathrm{m}^{2}\right]$ & $3.331 \times 10^{6}$ & $3.335 \times 10^{6}$ & $3.480 \times 10^{6}$ \\
\hline $\mathrm{h}\left[\mathrm{W} / \mathrm{m}^{2} / \mathrm{K}\right]$ at $\mathrm{T}_{\max }$ location & $5.927 \times 10^{4}$ & $6.00 \times 10^{4}$ & $6.307 \times 10^{4}$ \\
\hline$\Delta \mathrm{P}[\mathrm{Pa}]$ Through system & $5.98 \times 10^{5}$ & $5.99 \times 10^{5}$ & $5.60 \times 10^{5}$ \\
\hline Energy Conservation Error [\%] & 3.83 & 4.03 & 3.83 \\
\hline Mass Conservation Error [\%] & $4.77 \times 10^{-3}$ & $8.34 \times 10^{-3}$ & $5.96 \times 10^{-3}$ \\
\hline
\end{tabular}




\section{Chapter $7 \quad$ Conclusion}

This chapter summarizes the major results of this work and makes recommendations regarding further work associated with the thermal-hydraulic modeling of the High Flux Isotope Reactor.

\subsection{Results from 2-D Models}

It was shown that for the constant heat flux situation with both constant and variable fluid properties, the Seider-Tate correlation provided convection coefficient results very close to the COMSOL model results for the transfer of heat from a solid surface by the use of the low Reynold number k- $\varepsilon$ turbulent flow model. The Seider-Tate correlation produced a relative difference with the model Nusselt number of $1.2 \%$ to $4.4 \%$. As a result, the temperature of the surface of the generating solid was also closely matched by the Seider-Tate Nusselt modulus.

In the case of constant heat flux with constant fluid properties, the model produces surface temperature results that are higher than those produced by the other correlations, i.e. Gnielinski, Seider-Tate, and Hausen. This trend is inverted, however, with the use of variable fluid properties where the model surface temperature distribution is lowest in magnitude relative to the surface temperature distributions produced by the other correlations. It was found that the Gnielinski correlation produced the highest estimate of the surface temperature for the constant heat flux case with variable fluid properties.

For a surface exhibiting constant heat flux into a fluid where the material properties vary negligibly over the temperature range of interest, the Seider-Tate correlation produces estimates of the convection coefficient which translate into higher surface temperature distributions than the other correlations considered in this work. On the other hand, if the fluid properties vary significantly over the temperature range of interest with a constant surface heat flux, then the Gnielinski correlation will provide higher surface temperature results than the other correlations investigated herein. It should be borne in mind, however, that the use of constant fluid properties actually produced higher surface temperatures than the use of variable fluid properties. It was 
discussed that the use of constant fluid properties greatly reduces the amount of computational effort associated with the developed computational model. This reduction in effort is associated with the removal of the interpolation steps taken for the material properties at subsequent thermodynamic states.

Similar results were found for the distributed heat flux model. In the constant fluid property model, the surface temperature results associated with the model were larger in magnitude than the surface temperature results found from the correlations. It was observed that one could use either the Seider-Tate correlation or the Hausen correlation to closely reproduce the model surface temperature results in the constant fluid property case. Also, the Gnielinski correlation produced the lowest estimate for the surface temperature distribution. As a result, to provide higher estimates of the surface temperature, one should use either the Hausen correlation or the Seider-Tate correlation.

It was observed that the variable fluid property case produced the opposite trend, i.e. the Gnielinski correlation produced higher surface temperature results than the other correlations and the model. Also, the model associated with the variable fluid property case produced the lowest magnitude surface temperatures. For the variable fluid property case in conjunction with the distributed heat flux, the Hausen correlation most closely matched the model results over most of the range of the Reynolds numbers investigated here, i.e. 80,000 $\leq R e_{D} \leq 112,000$. On the higher end of the Reynolds number range, the Seider-Tate correlation was a better fit. The combination of a distributed power density in the generating material and variable fluid properties most closely matches the physics of the HFIR core system. As a result, it is recommended that the Gnielinski correlation be used to produce high estimates of the clad surface temperatures where conservative estimates are necessary.

\subsection{Results from the 3-D Models}

Both the Advanced Neutron Source Reactor Thermal Hydraulic Test loop model results and the results from the fuel defect models will be summarized here. 


\subsubsection{Advanced Neutron Source Reactor Thermal Hydraulic Test Loop}

As part of the validation effort of the COMSOL code, experimental data collected from the Advanced Neutron Source Reactor Thermal Hydraulic Test Loop (ANSR THTL) was used. In Chapter 5 it was demonstrated that the model of the ANSR THTL could reproduce the results of the experiment data considered in this work relatively well. This exercise with the ANSR THTL should instill confidence in the ability of the code to produce quality results within the parameters established for the ANSR THTL, i.e. high heat flux and large flow rates through long, narrow channels.

The ANSR THTL presents a unique experimental data set that has similar characteristics to that of the flow channels in the HFIR core, i.e. high surface heat flux and a long narrow coolant channel. The aspect ratio of the THTL coolant channel, length to thickness, is 399, which is only $16.9 \%$ less than that of the HFIR. Flow velocities through the THTL range from $62.34 \mathrm{fps}(19.00 \mathrm{~m} / \mathrm{s})$ to $88.58 \mathrm{fps}(27.00 \mathrm{~m} / \mathrm{s})$ which are also comparable to, albeit larger than, the flow velocity characteristic of the HFIR which, as previously stated, is approximately 52.00 fps $(15.85 \mathrm{~m} / \mathrm{s})$ at steady state operation. The average and peak heat fluxes produced in the THTL are stated to be $6 \mathrm{MW} / \mathrm{m}^{2}$ and $12 \mathrm{MW} / \mathrm{m}^{2}$, respectively [23] [24]. The average heat flux through the HFIR core is calculated to be $2.13 \mathrm{MW} / \mathrm{m}^{2}$. Therefore, with higher flow velocities and higher average heat fluxes, the THTL provides extreme cases, relative to HFIR conditions, with which to validate the commercial code COMSOL.

\subsubsection{Fuel Defect Analysis}

Two types of fuel plate defects were investigated, a lack of metallurgical bond, i.e. blister defect, and a segregation defect. The blister defect is physically characterized by a region with relatively low thermal conductivity, i.e. fission gas. The blister defect was incorporated into the model as an adiabatic disc located between the fuel and clad domains. The segregation defect is physically characterized as a local region with excess ${ }^{235} \mathrm{U}$ loading. The segregation defect was incorporated into the simulation environment as a cylinder coincident with the fuel meat with an increased thermal generation rate per unit volume relative to the surrounding fuel material. 
These defect models were compared with a nominal base case model in which no defects were incorporated. The location of the maximum surface temperature for the nominal case was used as the location for the defects. The logic of this strategy follows. The defects act to increase surface heat flux. The blister defect increases heat flux on the side of the plate opposite the defect geometry, i.e. the defect geometry was placed on the fuel contour side of the fuel plate and the heat flux was increased due to the defect on the uncontoured side of the fuel plate. The segregation defect increases the surface heat flux by a local increase in thermal generation rate per unit volume. An increase in surface heat flux results in an increase in surface temperatures, given that all other parameters remain unchanged, i.e. flow velocity, material properties, etc. The purpose of the fuel defect model is to investigate whether the surface temperature is comparable with the saturation temperature of the liquid which could result in a transition to two-phase flow. It was shown that the segregation defect resulted in the highest surface temperatures with a magnitude of $219.24^{\circ} \mathrm{F}(377.17 \mathrm{~K})$, however, the probability of either defect resulting in a local change of fluid phase is very low.

\subsection{Considerations for Future Use of Material in this Dissertation}

The material found herein presents models of the HFIR which, in a computational setting, represent the physics associated with the thermal hydraulic aspects of the device more so than the legacy codes of the past. That is not to say, however, that the legacy codes used in the development of the HFIR device are not amazing feats of ingenuity. Clearly, the developers of these codes had a very deep understanding of the physics associated with the design and use of the HFIR, but were limited by the availability of memory and computational processing power. However, it is the case that the code itself is poorly commented and difficult to modify with certainty. With the spectre of fuel conversion, i.e. HEU to LEU, modification of the legacy code is a daunting task indeed.

The work detailed in this dissertation provides a steady state methodology for the analysis of thermal hydraulic aspects of fuel defects. Further investigations should include geometric parameterization of the fuel defects. Necessary research includes determination of the 
maximum size for both the blister defect and the segregation defect which would result in a phase change of the fluid. This is especially important for the LEU fuel. Also, transient models regarding the growth of existing defects due to the irradiation of the fuel should be investigated. This research would provide useful information regarding fuel and fuel plate design to mitigate the effects of transient high temperature physics occurring at these locations. 
Bibliography 
[1] D. Chandler, R. T. Primm III and G. I. Maldonado, "Validating MCNP for LEU Fuel Design via Power Distribution Comparisons," Oak Ridge National Laboratory, Oak Ridge, TN., 2008.

[2] K. S. Krane, Introductory Nuclear Physics, Wiley \& Sons, 1988.

[3] D. G. Morris and M. W. Wendel, "High Flux Isotope Reactor System RELAP5 Input Model," Oak Ridge National Laboratory, Oak Ridge, TN., 1993.

[4] "National Nuclear Security Administration," [Online]. Available: http://nnsa.energy.gov. [Accessed 21 August 2012].

[5] "Reduced Enrichment for Research and Test Reactors," [Online]. Available: http://www.rertr.anl.gov. [Accessed 21 August 2012].

[6] D. E. Burkes, G. S. Mickum and D. M. Wachs, "Thermophysical Properties of U-10Mo Alloy," Idaho National Laboratory, 2010.

[7] G. Ilas and R. T. Primm III, " \{ Low Enriched Uranium Fuel Design with Two-Dimensional Grading for the High Flux Isotope Reactor," Oak Ridge National Laboratory, Oak Ridge, TN., 2010.

[8] NIST/ASME Steam Properties, Version 2.21, May 2004.

[9] F. P. Incropera, D. P. DeWitt, T. L. Bergman and A. S. Lavine, Fundamentals of Heat and Mass Transfer, Hoboken, NJ.: Wiley \& Sons, 2007.

[10] W. R. Gambill and R. D. Bundy, "HFIR Heat-Transfer Studies of Turbulent Water Flow in Thin Rectangular Channels," Oak Ridge National Laboratory, Oak Ridge, TN., 1961.

[11] R. D. Cheverton and T. M. Sims, "HFIR Core Nuclear Design," Oak Ridge National Laboratory, Oak Ridge, TN., 1971. 
[12] R. D. Cheverton and W. H. Kelley, "Experimental Investigation of HFIR Fuel Plate Deflections Induced by Temperature and Pressure Differentials," Oak Ridge National Laboratory, Oak Ridge, TN., 1968.

[13] N. Hilvety and T. G. Chapman, "HFIR Fuel Element Steady State Heat Transfer Analysis," Oak Ridge National Laboratory, Oak Ridge, TN., 1967.

[14] H. A. McLain, "HFIR Fuel Element Steady State Heat Transfer Analysis Revised Edition," Oak Ridge National Laboratory, Oak Ridge, TN., 1967.

[15] H. Hausen, Heat Transfer in Counter Flow, Parallel Flow, and Cross Flow, McGraw Hill, 1983.

[16] W. E. Thomas, "Bases for the Scram Setpoints, the Limiting Safety System Settings and the Safety Limits of the High Flux Isotope Reactor for Mode 1 Operation at 85MW(th)," Oak Ridge National Laboratory, Oak Ridge, TN., 1987.

[17] J. R. Kirkpatrick, "Calculations for HFIR Fuel Plate Non-Bonding and Fuel Segregation Uncertainty Factors," Oak Ridge National Laboratory, Oak Ridge, TN., 1990.

[18] G. E. Giles, "Advanced Neutron Source Reactor Thermal Analysis of Fuel Plate Defects," Oak Ridge National Laboratory, Oak Ridge, TN., 1995.

[19] G. Pastore and e. al., "Physica-based modelling of fission gas swelling and release in UO_2 applied to integral fuel rod analysis," Nuclear Engineering and Design, vol. 256, pp. 75-86, 2012.

[20] A. Boulore, C. Struzik and F. Gaudier, "Uncertainty and sensitivity analysis of the nuclear fuel thermal behavior," Nuclear Engineering and Design, vol. 253, pp. 200-210, 2012.

[21] G. Khvostov, K. Mikityuk and M. Zimmermann, "A model for fission gas release and gaseous swelling of the uranium dioxide fuel coupled with the FALCON code," Nuclear 
Engineering and Design, vol. 241, no. 8, pp. 2983-3007, 2011.

[22] D. Locke, "The Behavior of Defective Reactor Fuel," Nuclear Engineering and Design, vol. 21, no. 2, pp. 318-330, 1971.

[23] D. K. Felde and e. al., "Advanced Neutron Source Reactor Thermal-Hydraulic Test Loop Facility Description," Oak Ridge National Laboratory, Oak Ridge, TN., 1994.

[24] M. Siman-Tov and e. al., Static Flow Instability in Subcooled Flow Boiling in Parallel Channels, Kyoto, Japan: Presented at the 2nd International Conference on Multiphase Flow, 1995.

[25] D. Wilcox, Turbulence Modeling for CFD, La Cañada, CA: DCW Industries, 2006.

[26] H. Tennekes and J. Lumley, A First Course in Turbulence, Massachusetts: MIT Press, 1972.

[27] G. Batchelor, An Introduction to Fluid Dynamics, New York: Cambridge University Press, 1967.

[28] "CFD-Online," [Online]. Available: http://www.cfd-online.com/Wiki/Wilcox's_komega_model. [Accessed 02 November 2012].

[29] P. Davidson, Turbulence: An Introduction for Scientists and Engineers, New York, NY: Oxford University Press, 2004.

[30] COMSOL Help File - Turbulence Modeling.

[31] W. Kays and e. al., Convective Heat and Mass Transfer, New York, NY: McGraw-Hill, 2005.

[32] F. White, Viscous Fluid Flow, New York, NY: McGraw-Hill, 2006.

[33] A. Bejan, Convection Heat Transfer, 3rd. Ed., Hoboken, NJ.: John Wiley and Sons, 2004. 
[34] P. McCormack and L. Crane, Physical Fluid Dynamics, New York, NY.: Academic Press, Inc., 1973.

[35] T. Chung, Computational Fluid Dynamics, New York, NY.: Cambridge University Press, 2010.

[36] "Aerospace Specification Metals Incorporated," [Online]. Available: http://asm.matweb.com/search/SpecificMaterial.asp?bassnum=MA6061t6. [Accessed 14 November 2013].

[37] I. B. Celik and et.al., "Procedure for Estimation and Reporting of Uncertainty Due to Discretization in CFD Applications," Journal of Fluids Engineering, vol. 130, pp. 078001-1 to $078001-4,2008$.

[38] HFIR Inner Fuel Element Fuel Plate Loading Details, HFIR Drawing D-42114, Revision J, Oak Ridge, TN.: Oak Ridge National Laboratory, 2005.

[39] N. Todreas and M. Kazimi, Nuclear Systems I: Thermal Hydraulic Fundamentals, New York, NY: Taylor and Francis, 1990.

[40] HFIR Outer Fuel Element Fuel Plate Loading Details, HFIR Drawing D-42122 Revision J, Oak Ridge, TN.: Oak Ridge National Laboratory, 2005. 
Appendices 


\section{Appendix A}

The mesh plots from the NIST Steam Tables [8] for thermal conductivity, isobaric specific heat, dynamic viscosity, and mass density of light water are presented here in Figures A-1, A-2, A-3, and A-4, respectively. The abscissa of the plots are the absolute temperature, $\boldsymbol{T}$, and the thermodynamic pressure, $\boldsymbol{P}$. The range of temperatures over which each individual fluid property is reported is

$$
320.00 \mathrm{~K}<\boldsymbol{T}<394.26 \mathrm{~K} .
$$

The range of thermodynamic pressures over which each fluid property is reported is

$$
2.5 \mathrm{MPa}<\boldsymbol{P}<3.5 \mathrm{MPa} \text {. }
$$

These temperature and pressure ranges are consistent with the nominal operation of the HFIR core system. 


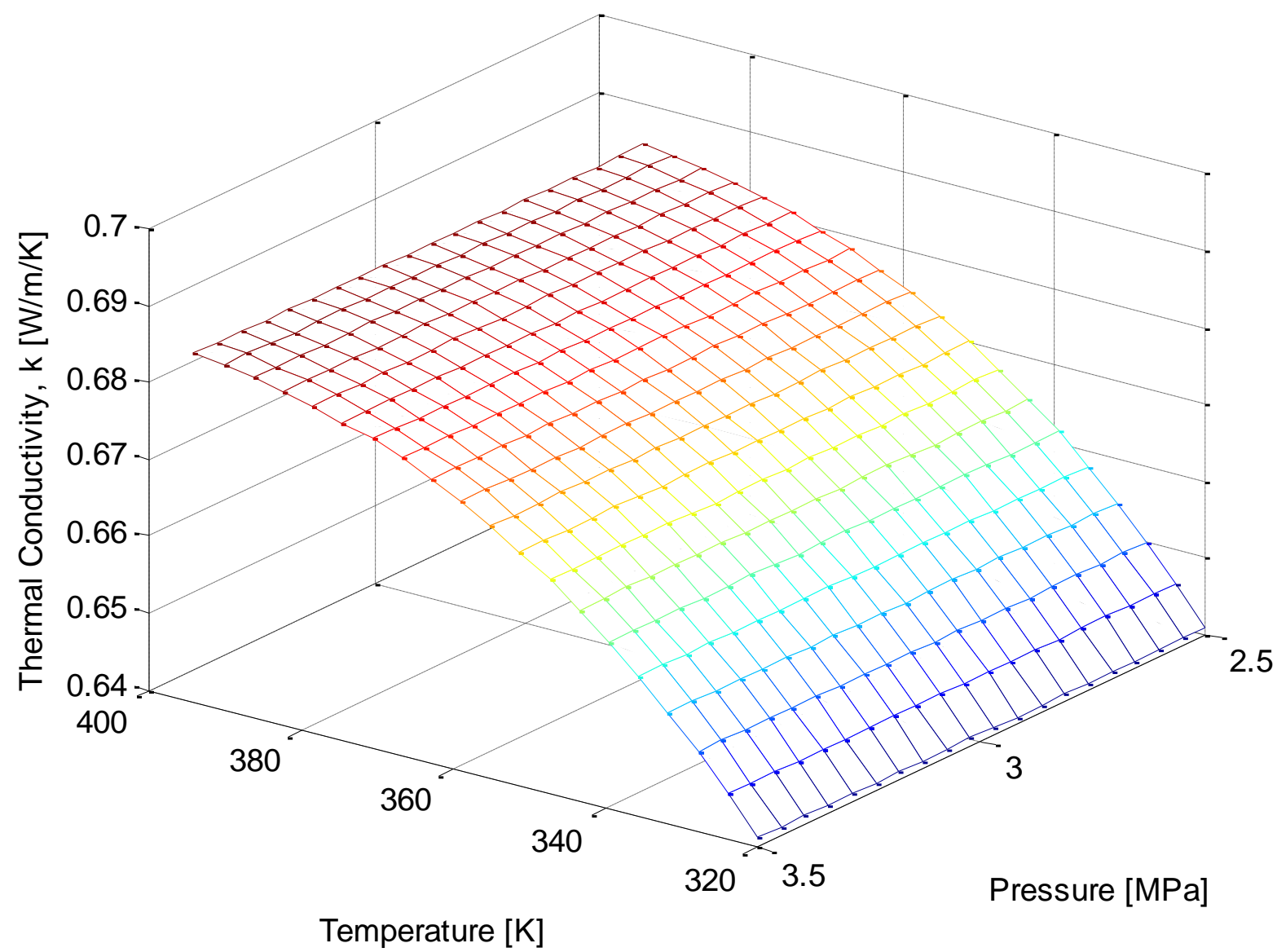

Figure A-1. Thermal Conductivity of Water as a Function of the Thermodynamic State. 


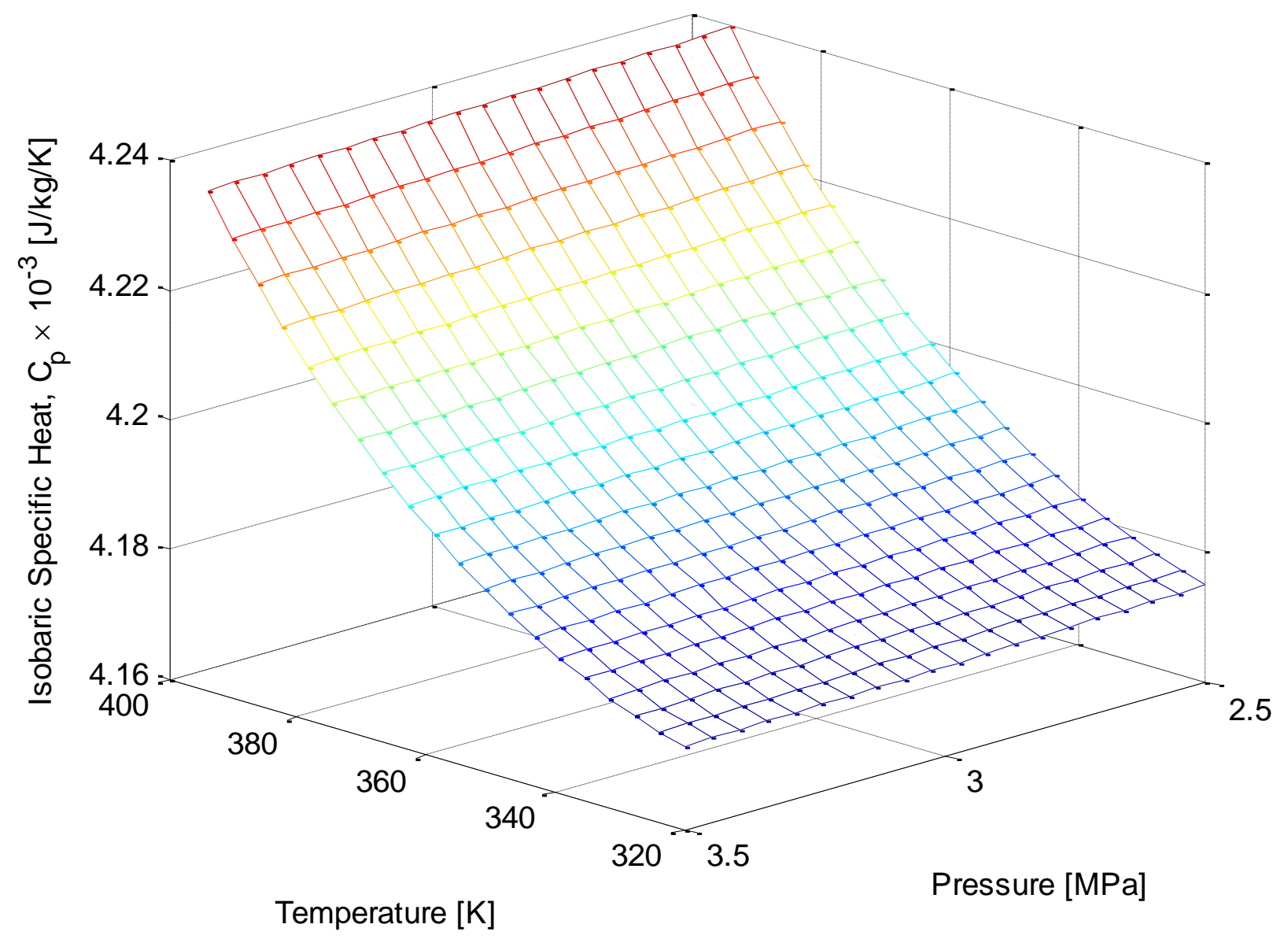

Figure A-2. Isobaric Specific Heat of Water as a Function of the Thermodynamic State. 


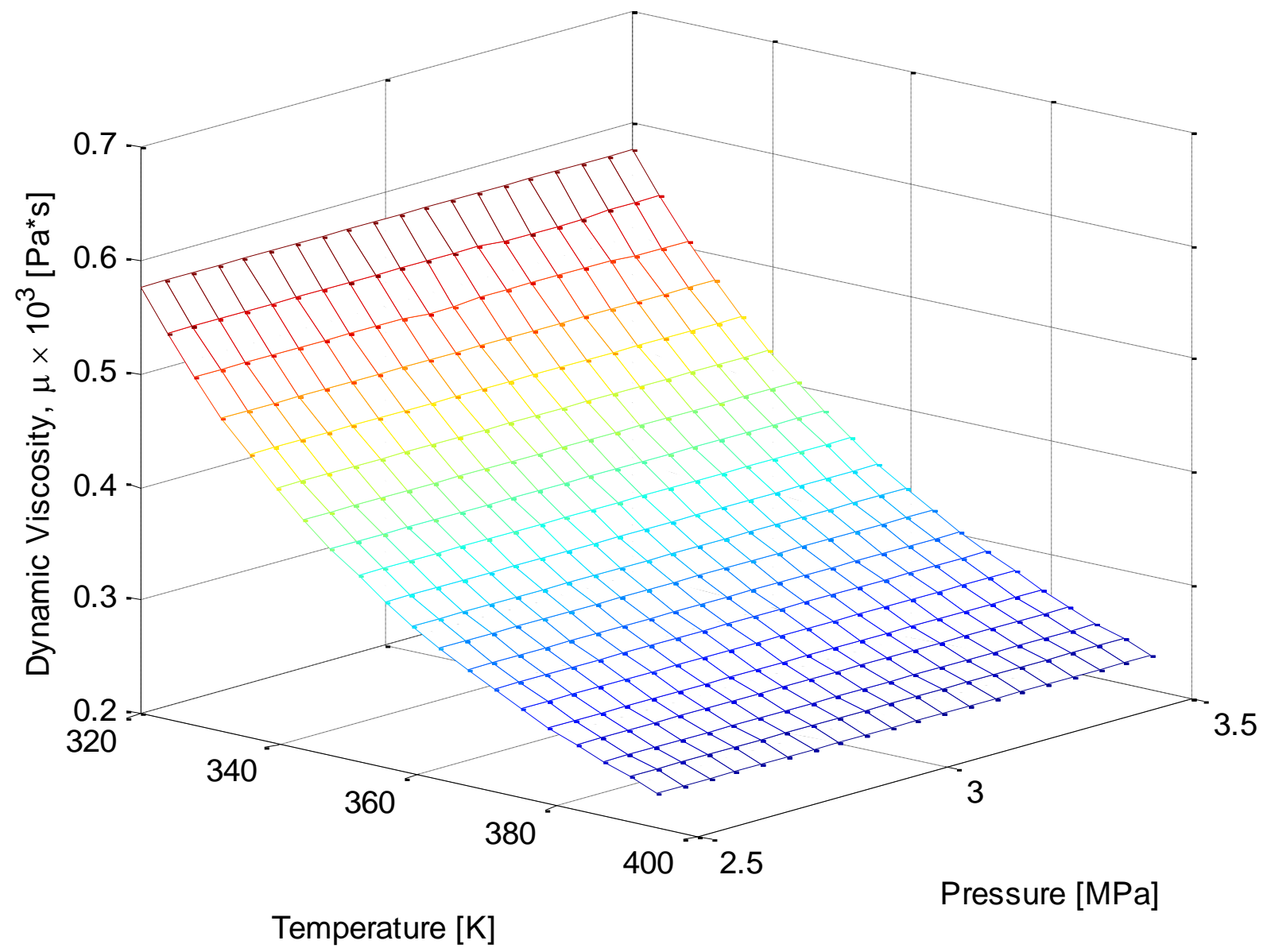

Figure A-3. Dynamic Viscosity of Water as a Function of the Thermodynamic State. 


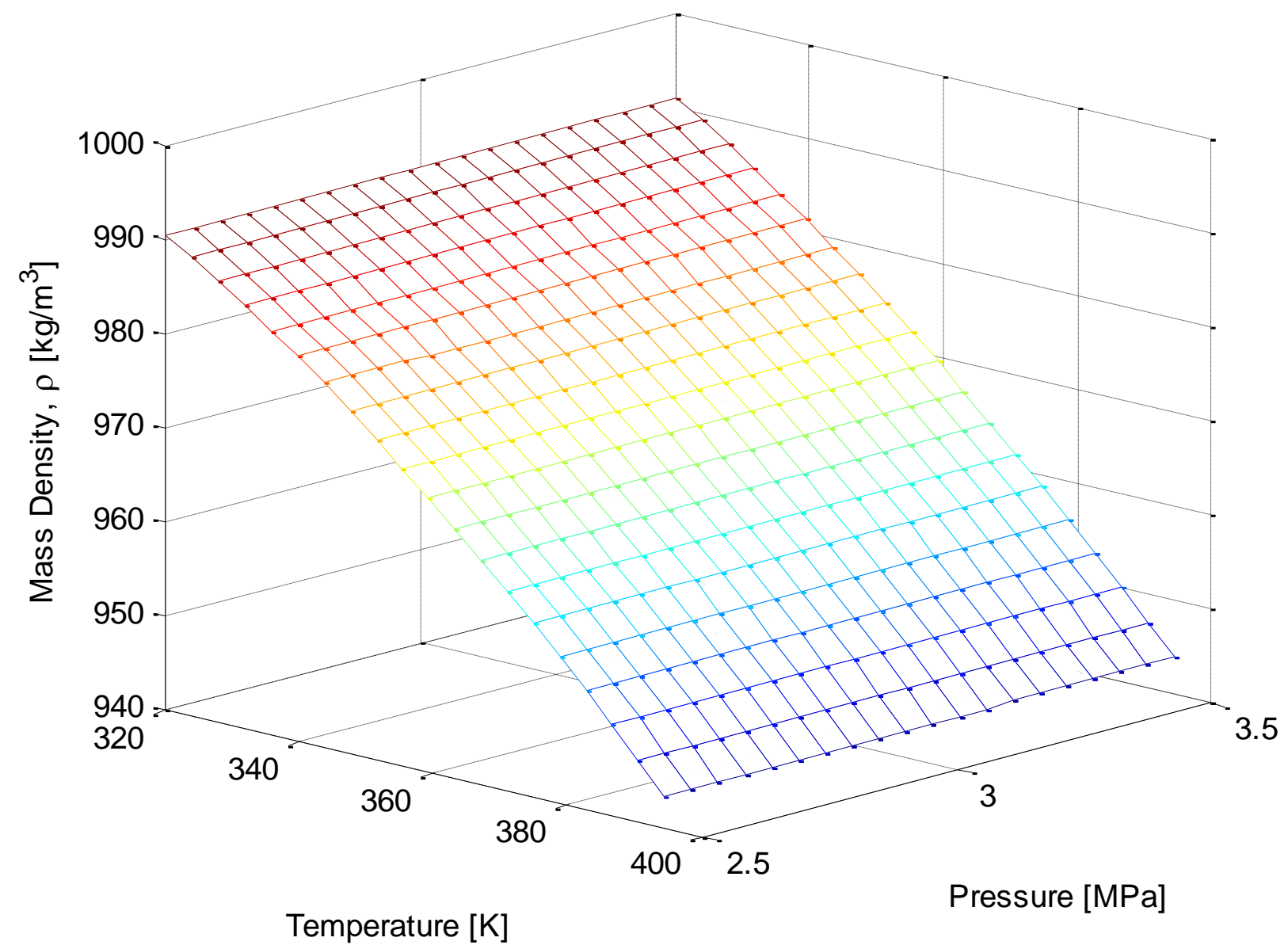

Figure A-4. Mass Density of Water as a Function of the Thermodynamic State. 


\section{Appendix B}

The determination of the fuel volumes within a single inner and outer fuel plate are outlined here. The contours of the fuel meat for both the inner fuel plate [38] and the outer fuel plate [40] are defined by point locations that reside at the surface of the fuel meat. The drawings use a relative scale for the point locations and therefore a conversion to length was necessary. These points were entered as input to MATLAB.

Cubic spline interpolation of the fuel meat contour point locations was performed in the MATLAB environment. The results of the interpolation are shown in Figure B-1 and Figure B2. The first and last points represent the boundaries of the fuel meat geometry. The spline contours were then subject to Simpson's quadrature to evaluate the area under the spline. The resultant area was then extruded through 20.0 inches $(0.508 \mathrm{~m})$, i.e. nominal fuel meat length, resulting in the volume of the fuel meat within the individual fuel plates, i.e. inner and outer fuel plates. 


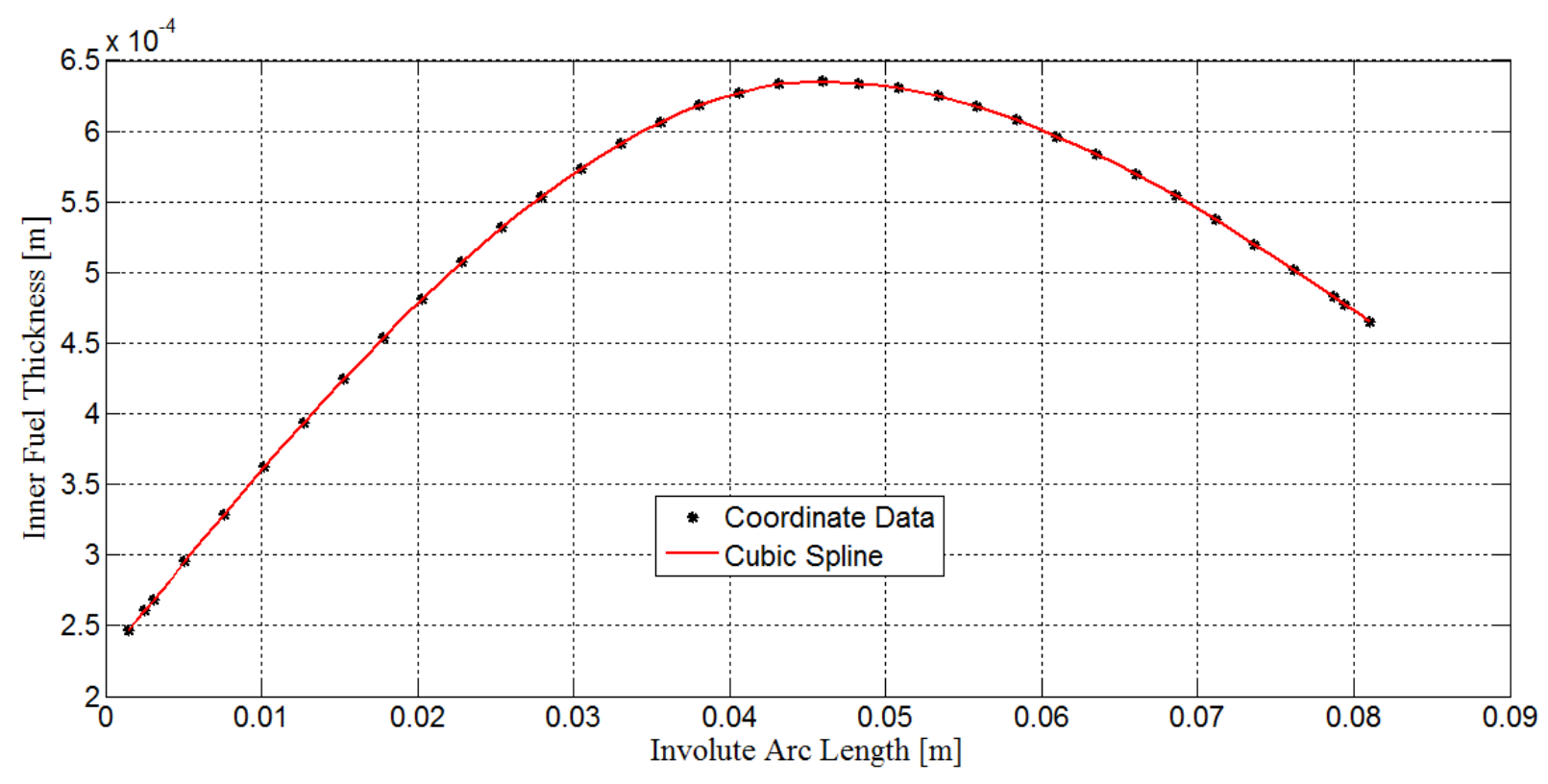

Figure B-1. Cubic Spline Interpolation of the Inner Fuel Element Fuel Plate Loading.

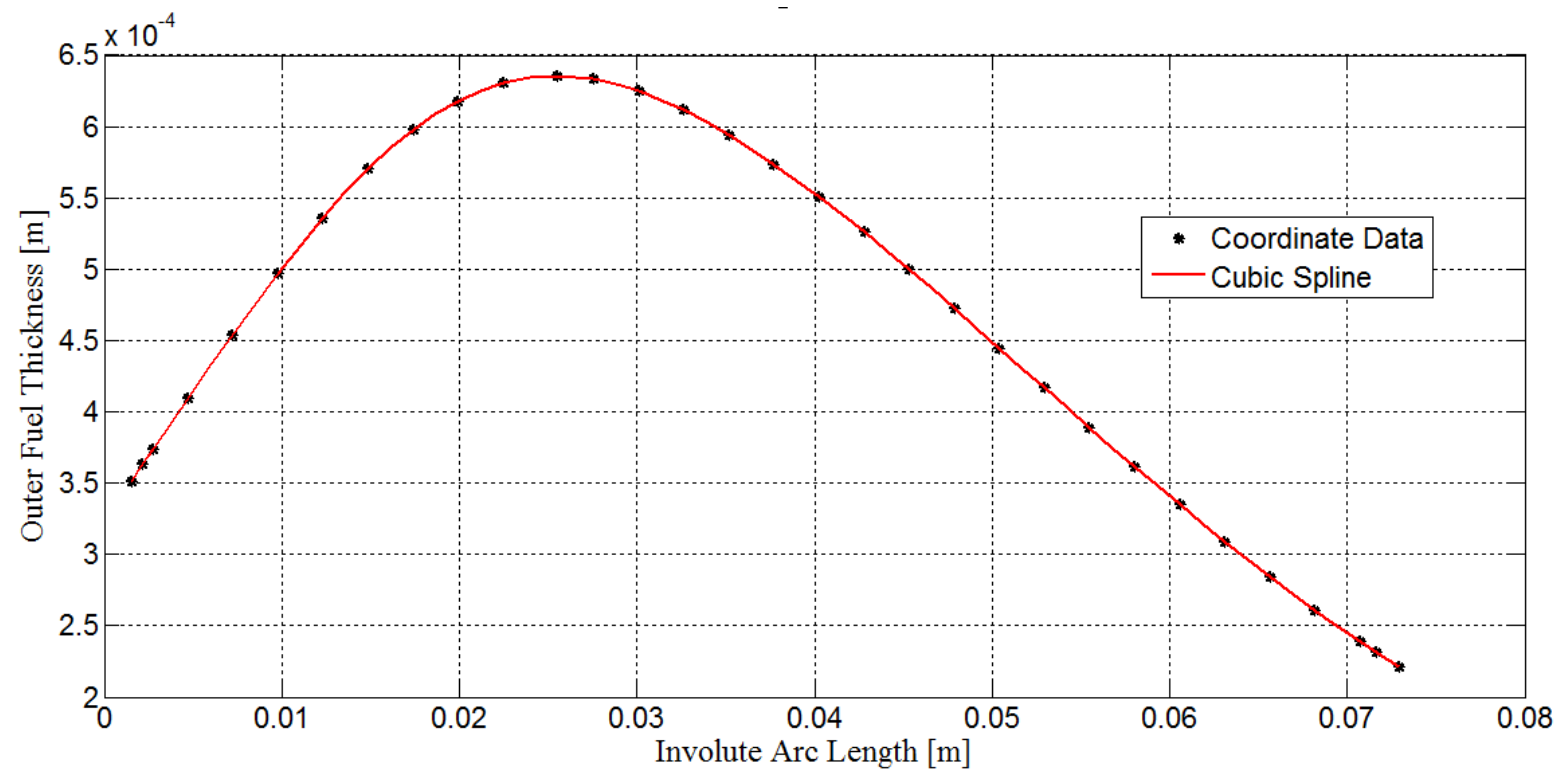

Figure B-2. Cubic Spline Interpolation of the Outer Fuel Element Fuel Plate Loading. 


\section{INTERNAL DISTRIBUTION}

1. D. H. Cook (dhc@ornl.gov)

2. J. D. Freels (freelsjd@ornl.gov)

3. G. Ilas (ilasg@ornl.gov)

4. P. K. Jain (jainpk@ornl.gov

5. D. G. Renfro (renfrodg@ornl.gov)

6. K. A. Smith (smithka@ornl.gov)

7. G. L. Yoder Jr. (yodergljr@ornl.gov)

8. D. Chandler (chandlerd@ornl.gov)

9. $\quad$ E. L. Popov (popove@ornl.gov)

10. M. W. Kohring (kohringmw@ornl.gov)

11. ORNL/RRD Document Control (phillipsth@ornl.gov)

\section{EXTERNAL DISTRIBUTION}

12. I. T. Bodey, Savannah River Remediation, LLC., DWPF Mechanical Engineering Building: 704-25S Room 7 (Isaac.Bodey@srs.gov)

13. R. V. Arimilli, Professor, Mechanical, Aerospace and Biomedical Engineering Department, 414 Dougherty Engineering Building, The University of Tennessee, Knoxville, Tennessee 37996-2210 (arimilli@utk.edu)

14. E. Fontes, COMSOL AB, Tegnergatan 23, SE-111 40, Stockholm, Sweden (ed@comsol.com)

15. W. F. Jones, Idaho National Laboratory, P.O. Box 1625, Idaho Falls, ID 83415-3750 (warren.jones@inl.gov)

16. K. T. Lowe, Bettis Atomic Power Laboratory, Bechtel Bettis, Inc, Mail Stop 36E, P. O. Box 79, West Mifflin, PA, 15122 (lowekt@bettis.gov)

17. A. E. Ruggles, Department of Nuclear Engineering, 315 Pasqua Engineering Building, Knoxville, TN 37996-2300 (aruggles@utk.edu)

18. B. Sjodin, COMSOL, Inc., New England Executive Park, Suite 350, Burlington, MA 01803 (bjorn@comsol.com)

19. J. G. Stevens, Research and Test Reactors Department Manager, Nuclear Engineering Division, Argonne National Laboratory, 9700 S Cass Avenue, Bldg 208, Argonne, IL 60439 (johnstevens@anl.gov)

20. W. R. Marcum, Assistant Professor, Nuclear Engineering \& Radiation Health Physics, Oregon State University (marcumw@engr.orst.edu)

21. G. L. Solbrekken, Assistant Professor, Mechanical and Aerospace Engineering, E2411A Lafferre Hall, Columbia, MO, 65211 (SolbrekkenG@missouri.edu)

22. A. M. Tentner, Nuclear Engineering Division, Argonne National Laboratory, $9700 \mathrm{~S}$ Cass Avenue, Bldg 208, Argonne, IL 60439 (tentner@anl.gov)

23. K. Ekici, Assistant Professor, Graduate Program Director, Mechanical, Aerospace and Biomedical Engineering Department, 315 Perkins Hall, 415 Dougherty Engineering Building, The University of Tennessee, Knoxville, Tennessee 37996-2030 (kici@utk.edu) 